\title{
Complete form factors in Yang-Mills from unitarity and spinor helicity in six dimensions
}

\author{
Manuel Accettulli Huber $\odot,{ }^{*}$ Andreas Brandhuber $\odot,{ }^{\dagger}$ Stefano De Angelis $\odot,{ }^{\ddagger}$ and Gabriele Travaglini $\odot^{\S}$ \\ Centre for Research in String Theory, School of Physics and Astronomy, \\ Queen Mary University of London, Mile End Road, London E1 4NS, United Kingdom
}

(Received 22 October 2019; published 6 January 2020)

\begin{abstract}
We present a systematic procedure to compute complete, analytic form factors of gauge-invariant operators at loop level in pure Yang-Mills. We consider applications to operators of the form $\operatorname{Tr} F^{n}$ where $F$ is the gluon field strength. Our approach is based on an extension to form factors of the dimensional reconstruction technique, in conjunction with the six-dimensional spinor-helicity formalism and generalized unitarity. For form factors this technique requires the introduction of additional scalar operators, for which we provide a systematic prescription. We also discuss a generalization of dimensional reconstruction to any number of loops, both for amplitudes and form factors. Several novel results for one-loop minimal and nonminimal form factors of $\operatorname{Tr} F^{n}$ with $n>2$ are presented. Finally, we describe the Mathematica package spinorHelicity6D, which is tailored to handle six-dimensional quantities written in the spinorhelicity formalism.
\end{abstract}

DOI: 10.1103/PhysRevD.101.026004

\section{INTRODUCTION}

The aim of this paper is to construct complete, analytic form factors of gauge-invariant operators at one loop. In supersymmetric theories, four-dimensional unitarity $[1,2]$ is sufficient to obtain complete answers for amplitudes at one loop. Without supersymmetry or for form factors of non-protected operators this is no longer the case because of the appearance of rational contributions. In the amplitude context this problem has been addressed in different ways. In one approach, one makes use of factorization to establish a recursion relation that allows to reconstruct rational terms $[3,4]$ (see $[5,6]$ for recent elegant applications to two-loop amplitudes in pure YangMills). Another approach is to shift the dimensionality of internal states in the loop away from four dimensions $[7,8]$ where rational terms acquire a singularity which can then be detected using unitarity cuts. Multiple cuts have also been used efficiently in this context [9-11]. This method requires that the internal lines, corresponding to virtual particles, are kept in $d$ dimensions, while

\footnotetext{
*m.accettullihuber@qmul.ac.uk

†a.brandhuber@qmul.ac.uk

*s.deangelis@qmul.ac.uk

§.travaglini@qmul.ac.uk
}

Published by the American Physical Society under the terms of the Creative Commons Attribution 4.0 International license. Further distribution of this work must maintain attribution to the author(s) and the published article's title, journal citation, and DOI. Funded by SCOAP ${ }^{3}$. momenta and polarization vectors of external particles live in four dimensions.

Having the internal particles in arbitrary, noninteger dimensions introduces complications, since tree amplitudes are no longer simple and the power of the spinorhelicity formalism is lost. A solution to this problem is offered by "dimensional reconstruction" [12-16]. In this approach, one investigates the dependence of the loop amplitudes on the dimensionality of spacetime, which turns out to be polynomial in pure Yang-Mills theory. Then one computes the amplitudes with virtual particles kept in integer dimension $d>4$ to fix the coefficients in the polynomial by interpolation, which leads by analytic continuation to an expression valid for any non-integer dimension $d$. The dimensional reconstruction approach can also be effectively combined with the spinor-helicity formalism in six dimensions of [17], which allows for compact expressions of the on-shell building blocks. At higher loops, these techniques were used in [18] to derive the five-point all-plus gluon amplitude integrand in pure Yang-Mills, while a generalization to incorporate fermions was carried out in [19]. Recent numerical as well as analytical results for arbitrary helicity configurations of five partons were derived in [20-22]. In this framework, a systematic prescription for complete form factors, including rational terms, is still missing, and bridging this gap is one of the main goals of this paper.

A form factor $F_{\mathcal{O}}(1, \ldots, n ; q)$ is defined as the overlap of an $n$-particle state and the state produced by an operator $\mathcal{O}(x)$ acting on the vacuum: 


$$
\begin{aligned}
& \int \mathrm{d}^{4} x e^{-i q \cdot x}\langle 1, \ldots, n|\mathcal{O}(x)| 0\rangle \\
& =(2 \pi)^{4} \delta^{(4)}\left(q-\sum_{i=1}^{n} p_{i}\right) F_{\mathcal{O}}(1, \ldots, n ; q) .
\end{aligned}
$$

Notable examples of form factors include the form factor of the hadronic electromagnetic current with an external hadronic state, which feature in the $e^{+} e^{-} \rightarrow$ hadrons and deep inelastic scattering matrix elements, and as the form factor of the electromagnetic current, which computes the (electron) $g-2$. The form factors which will be considered in this paper are related to scattering processes of the Higgs boson and many gluons. In the large top-quark mass approximation, these can be described by an effective theory obtained by integrating out the top quark in QCD. This generates an infinite series of higher-dimensional interactions of the Higgs with the gluon field strength and its derivatives, in addition to couplings to light quarks. More precisely, this effective Lagrangian reads

$$
\mathcal{L}_{\text {eff }}=\hat{C}_{0} \mathcal{O}_{0}+\frac{1}{m_{t}^{2}} \sum_{i=1}^{4} \hat{C}_{i} \mathcal{O}_{i}+\mathcal{O}\left(\frac{1}{m_{t}^{4}}\right)
$$

where the leading-order term in the expansion is $\mathcal{O}_{0}:=$ $H \operatorname{Tr} F^{2}$ [23-26], $\mathcal{O}_{i}, i=1, \ldots, 4$ are dimension-7 operators made of gluon field strengths and covariant derivatives [27-30], $m_{t}$ is the mass of the top quark, and $\hat{C}_{0}, \hat{C}_{i}$ are Wilson coefficients. ${ }^{1}$ After Wick-contracting the Higgs field, what is left to compute is precisely a form factor of partons in the theory of interest, which we will take to be pure Yang-Mills. It is also interesting to note that at zero momentum transfer, i.e., $q=0$ in (1.1), the form factor of an operator $\mathcal{O}(x)$ represents the correction to the scattering amplitude due to the inclusion of a new local interaction proportional to $\mathcal{O}(x)$. On the other hand, in the study of Higgs + gluon processes one replaces $q^{2}$ with the squared mass of the Higgs $m_{\mathrm{H}}^{2}$ to obtain the amplitudes relevant for this process.

In this paper we will apply the approach discussed so far to form factors of operators of the form $\operatorname{Tr} F^{n}$, for $n=2,3$, 4 , both for minimal and nonminimal form factors up to four external gluons. Modern amplitude techniques were applied to form factors of $\operatorname{Tr} F^{2}$, which compute the leading contribution to Higgs + multigluon amplitudes in the effective Lagrangian approach, including MHV diagrams [31,32] at tree level [33,34] and one loop [35], and a combination of one-loop MHV diagrams and recursion relations [36]. Recent work [37-41] addressed the computation of the four-dimensional cut-constructible part of

\footnotetext{
${ }^{1}$ The Wilson coefficients are proportional to $1 / v$, where $v$ is the Higgs field vacuum expectation value. Their precise form will be of no relevance for this paper.
}

Higgs+multi-gluon scattering from operators of mass dimension seven using generalized unitarity [42,43] applied to form factors $[39,40,44-55]$. The key point of this work is that we extend dimensional reconstruction to any form factor of operators involving vector fields, which requires the subtraction of form factors of an appropriate class of scalar operators that we identify. Along the way we have also found a generalization of this procedure to any loop order, for amplitudes and form factors.

Loop-level calculations are inherently difficult, and no matter how effective the method or how simple the formalism are, sooner or later the complexity of the problems one wishes to tackle will require the use of computer software to deal with the algebra. For this reason we have written a Mathematica package that can handle quantities and perform computations in the six-dimensional spinor-helicity formalism. This package has been inspired by implementations of the analogous four-dimensional formalism in [56-58] and features analytic as well as numerical tools.

The rest of the paper is organized as follows. In Sec. II we review the dimensional reconstruction technique at one loop and generalize it to form factors involving vector fields. We also discuss its generalization to any number of loops, which for one and two loops is in agreement with known results. In Sec. III we study tree-level form factors for a wide class of operators involving field strengths in four and six dimensions. These quantities are needed in the one-loop unitarity-based calculations of Sec. IV. There, we begin by reproducing the well-know one-loop form factors for $\operatorname{Tr} F^{2}$ with two and three external gluons. Then we prove that the minimal form factor for $\operatorname{Tr} F^{3}$ has no rational terms, as argued in the literature. Finally, we calculate for the first time the nonminimal one-loop form factor for $\operatorname{Tr} F^{3}$ and the minimal form factor for $\operatorname{Tr} F^{4}$ with different helicity configurations. We also generalize some of these results for a class of form factors of the $\operatorname{Tr} F^{n}$ operators. A few appendices complete the paper. In the first three we review the spinor-helicity formalism in four and six dimensions, as well as the structure of six-dimensional tree amplitudes. Appendix D contains detailed calculations of non-minimal tree-level form factors used as building blocks of loop amplitudes, while Appendix E lists some useful results on integral functions. Finally, Appendix F contains a short description of the spinorHelicity6D Mathematica package we have used in our numerical calculations, focusing on the functions required to replicate our results.

\section{THE DIMENSIONAL RECONSTRUCTION TECHNIQUE}

In the first part of the section, we look at the one-loop case from a different perspective which lends itself to a systematic generalization to form factors. The new viewpoint we adopt presents also a much desirable advantage: it disentangles the number of dimensions in which 
amplitudes need to be computed from the loop order. This feature allows for a natural generalization to any loop order, for both amplitudes and form factors, which will be discussed in the second part of the section.

\section{A. One-loop dimensional reconstruction}

The first step in our study is to identify the dependence of the loop amplitude on the dimensionality of the spacetime. In the literature, a common procedure is to distinguish the two sources of this dependence:

(i) the first is the number of spin-eigenstates, which is a function of the dimension of the spacetime $d_{s}$ (for example, gluons have $d_{s}-2$ spin degrees of freedom (d.o.f.));

(ii) the second is the integration over the loop momentum, which lives in a $d$-dimensional space.

Specifically, in the following we consider pure Yang-Mills theory

$$
\mathcal{L}_{d_{s}}=-\frac{1}{4}\left(F_{\mu \nu}^{a} F^{a \mu \nu}\right)(x),
$$

where $A^{a \mu}$ is a vector in a spacetime of dimension $d_{s}$ and $x$ is defined on a spacetime of dimension $d>4$.

As we mentioned earlier, we are interested in calculating amplitudes (and form factors) involving four-dimensional external gluons. At one loop it is possible to write a general amplitude as

$$
\mathcal{A}_{\left(d_{s}, d\right)}^{(1)}\left(\left\{p_{i}, h_{i}\right\}\right)=\int \frac{\mathrm{d}^{d} l}{(2 \pi)^{d}} \frac{\mathcal{N}^{d_{s}}\left(\left\{p_{i}, h_{i}\right\}\right)}{\prod_{i} D_{i}},
$$

where $\mathcal{N}^{d_{s}}\left(\left\{p_{i}, h_{i}\right\}\right)$ depends on $d_{s}$ through the number of spin eigenstates and on $d$ through the loop momentum. However, since all external momenta are four-dimensional, the additional components of the loop momentum enter the amplitude only through its square, which can always be written as

$$
l^{2}=l_{0}^{2}-l_{1}^{2}-l_{2}^{2}-l_{3}^{2}-\sum_{i=4}^{d-1} l_{i}^{2}:=\left(l^{(4)}\right)^{2}-\mu^{2} .
$$

The dependence of the amplitude on $\mu^{2}$ manifests itself in a number of additional integrals with nontrivial numerators, which have to be added to the usual master integral basis. These integrals have the form:

$$
\int \frac{\mathrm{d}^{d} l}{(2 \pi)^{d}} \frac{\mu^{2 p}}{D_{1} \cdots D_{n}}:=I_{n}^{d}\left[\mu^{2 p}\right],
$$

which can be evaluated as ordinary integrals, but in higher dimensions [8]. The presence of these integrals cannot be probed using four-dimensional unitarity cuts.

Consider now the explicit dependence of the amplitude on $d_{s}$. One-loop amplitudes involving only bosons are linear in $d_{s}$, because it appears only in a closed loop of contracted metric tensors coming from vertices and propagators. Consequently, in order to determine the dependence of the amplitude on $d_{s}$, only two constants need to be fixed and these can be obtained by interpolation. Thus it is sufficient to compute the amplitude in two integer dimensions, for example $d_{0}$ and $d_{1}=d_{0}+1$, and then write the analytic continuation to four dimensions in the four dimensional helicity (FDH) scheme $[59,60]$. The result of the interpolation is given by [12]:

$$
\mathcal{A}_{(4, d)}^{(1)}=\left(d_{1}-4\right) \mathcal{A}_{\left(d_{0}, d\right)}^{(1)}-\left(d_{0}-4\right) \mathcal{A}_{\left(d_{1}, d\right)}^{(1)} .
$$

By definition $d$ are the dynamical dimensions of the theory and we can always choose $d_{0} \geq d$. By doing so we can consider the extra dimension in the $d_{1}$-dimensional space as non-dynamical. Then a $d_{1}$-dimensional gluon behaves as a $d_{0}$-dimensional one plus a real scalar $A^{a \mu}=\left(A^{a \hat{\mu}}, \phi^{a}\right)$, and the Lagrangian of the system reads ${ }^{2}$

$\mathcal{L}_{d_{i}}=-\frac{1}{4} F_{\mu \nu}^{a} F^{a \mu \nu}=-\frac{1}{4} F_{\hat{\mu} \hat{\nu}}^{a} F^{a \hat{\mu} \hat{\nu}}+\frac{1}{2} D_{\hat{\mu}} \phi^{a} D^{\hat{\mu}} \phi^{a}$,

where the hatted quantities refer to $d_{0}$-dimensional Lorentz indices. From the Lagrangian (2.6) we arrive at the conclusion that the one-loop $d_{i}$-dimensional amplitude $\mathcal{A}_{\left(d_{i}, d\right)}$ can be expressed as the sum of two contributions: the first contribution is given by the equivalent one-loop gluon amplitude with internal particles living in $d_{0}$ dimensions $\mathcal{A}_{\left(d_{0}, d\right)}$; the second one, denoted in the following as $\mathcal{A}_{(d)}^{S}$, takes into account also scalar interactions coming from the second term on the right-hand side of (2.6). It is also important to stress that $\mathcal{A}_{(d)}^{S}$ is a gauge-invariant quantity in its own right. As a result of these observations, (2.5) can be written as:

$$
\mathcal{A}_{(4, d)}^{(1)}=\mathcal{A}_{\left(d_{0}, d\right)}^{(1)}-\left(d_{0}-4\right) \mathcal{A}_{(d)}^{S} .
$$

Since we are considering only the one-loop order, it is easy to see that $\mathcal{A}_{(d)}^{S}$ can be obtained by trading the gluon loop for a scalar loop.

Up to some additional considerations, the above discussion holds true for form factors as well, and so does (2.7). In particular, the scalar quantity that we have to subtract from the form factor with $d_{0}$-dimensional internal gluons is obtained by trading the gluon loop with a scalar one. However, in contradistinction with the amplitude case, there are two sources for scalars when we are dealing with form factors. Inside the loop, one can have scalars coupled to gluon lines coming from terms of the form $\frac{1}{2} D_{\mu} \phi^{a} D^{\mu} \phi^{a}$ in the dimensionally-reduced Lagrangian (as in the case of

\footnotetext{
${ }^{2}$ The fields are nondynamical in the $d_{1}$-dimensional direction of the space-time, thus we can set $\partial_{d_{0}} A^{a \mu}=0$ and $\partial_{d_{0}} \phi^{a}=0$ $\left(\partial_{d_{0}}=\partial_{d_{1}-1}\right)$.
} 
amplitudes), but also scalars coming from the operator inserted in the form factor. This procedure will be clear in the calculation of the nonminimal $\operatorname{Tr} F^{2}$ form factor, described in Sec. IV B, where we will emphasize the role of these two distinct contributions (see also [16]).

Finally, what we need is to identify the scalar operator. The procedure we follow is reminiscent of dimensional reduction, which for the operator $\operatorname{Tr} F^{2}$ was performed in (2.6). From this new point of view, the generalization of the dimensional reconstruction technique to other operators is straightforward. In particular, for the only two operators with mass-dimension six involving solely gluons, namely $\operatorname{Tr}(D F)^{2}$ and $\operatorname{Tr} F^{3}$, the scalar contribution comes from

$$
D_{\mu} F_{\nu \rho}^{a} D^{\mu} F^{a \nu \rho} \mapsto D_{\mu} D_{\nu} \phi^{a} D^{\mu} D^{\nu} \phi^{a},
$$

and

$$
f^{a b c} F^{a \mu}{ }_{\nu} F^{b \nu}{ }_{\rho} F^{c \rho}{ }_{\mu} \mapsto f^{a b c} D_{\mu} \phi^{a} D_{\nu} \phi^{b} F^{c \mu \nu},
$$

where scalar operators associated to each operator come from the dimensional reduction from $d_{1}$ to $d_{0}$. On the other hand for the $\operatorname{Tr} F^{4}$ operator, which we will consider later in this paper, at one-loop we get

$$
\operatorname{Tr} F_{\nu}^{\mu} F_{\rho}^{\nu} F_{\sigma}^{\rho} F_{\mu}^{\sigma} \mapsto \operatorname{Tr} D_{\mu} \phi D_{\nu} \phi F_{\rho}^{\nu} F^{\rho \mu},
$$

where in the last equation the trace is in color space. ${ }^{3}$ The proportionality coefficients are still to be fixed and we will give the right prescription for them within the full tree-level calculation in Sec. III.

\section{B. An $L$-loop generalization}

The arguments leading to (2.5) can be extended to arbitrary loop order. Considering pure Yang-Mills theory, any $L$-loop amplitude can be written as a degree $L$ polynomial in the dimension $d_{s}{ }^{4}$,

$$
\mathcal{A}_{\left(d_{s}, d\right)}^{(L)}=\sum_{i=0}^{L}\left(d_{s}-4\right)^{i} \mathcal{K}_{i}
$$

where $\mathcal{K}_{i}$ are quantities to be determined. In particular, note that the four-dimensional amplitude in the FDH scheme $[59,60]$ coincides with the zero-degree coefficient: $\mathcal{K}_{0}=\mathcal{A}_{(4, d)}^{(L)}$. In order to find the coefficients $\mathcal{K}_{i}$, we can interpolate the polynomial in $L+1$ distinct integer dimensions $d_{i}>4$. Writing the problem in matrix form, one has

$$
\left(\begin{array}{c}
\mathcal{A}_{\left(d_{0}, d\right)}^{(L)} \\
\mathcal{A}_{\left(d_{1}, d\right)}^{(L)} \\
\vdots \\
\mathcal{A}_{\left(d_{L}, d\right)}^{(L)}
\end{array}\right)=\left(\begin{array}{ccccc}
1 & \left(d_{0}-4\right) & \left(d_{0}-4\right)^{2} & \cdots & \left(d_{0}-4\right)^{L} \\
1 & \left(d_{1}-4\right) & \left(d_{1}-4\right)^{2} & \cdots & \left(d_{1}-4\right)^{L} \\
\vdots & & & & \vdots \\
1 & \left(d_{L}-4\right) & \left(d_{L}-4\right)^{2} & \cdots & \left(d_{L}-4\right)^{L}
\end{array}\right)\left(\begin{array}{c}
\mathcal{K}_{0} \\
\mathcal{K}_{1} \\
\vdots \\
\mathcal{K}_{L}
\end{array}\right),
$$

where we recognize the Vandermonde matrix. Inverting this matrix, it is possible to express the $\mathcal{K}_{i}$ as functions of the higher-dimensional amplitudes $\mathcal{A}_{\left(d_{i}, d\right)}^{(L)}$ for $i=0, \ldots, L$. In particular $\mathcal{K}_{0}$, which is the four-dimensional amplitude we are interested in, can be written as

$\mathcal{A}_{(4, d)}^{(L)}=\mathcal{K}_{0}=\prod_{j=0}^{L}\left(d_{j}-4\right) \sum_{i=0}^{L} \frac{1}{\left(d_{i}-4\right)} \prod_{\substack{k=0 \\ k \neq i}}^{L} \frac{1}{\left(d_{k}-d_{i}\right)} \mathcal{A}_{\left(d_{i}, d\right)}^{(L)}$.

We can always choose $d_{0}>4$ to be the smallest dimension among the $d_{i}$ 's, and we also know that at most

\footnotetext{
${ }^{3}$ We emphasize that (2.10) is exactly the scalar operator up to an overall factor, which has still to be fixed. In particular, the two scalars in the previous operator have to be adjacent, because the gluon operator involves only contractions between adjacent field strength.

${ }^{4}$ As already mentioned, the $d_{s}$ dependence comes from traces of $\eta$ tensors, and there can be at most $L$ closed loops leading to such a trace.
}

$d$ dimensions are dynamical, with $4<d \leq d_{0}$. Then, we can write the Lagrangian of pure Yang-Mills theory in $d_{i}>d_{0}$ dimensions as:

$$
\begin{aligned}
\mathcal{L}_{d_{i}}= & -\frac{1}{4} F_{\mu \nu}^{a} F^{a \mu \nu}+\frac{1}{2} \sum_{i=1}^{d_{i}-d_{0}} D_{\mu} \phi_{i}^{a} D^{\mu} \phi_{i}^{a} \\
& -\frac{\lambda}{2} f^{a b c} f^{a d e} \sum_{\substack{i, j=1 \\
j>i}}^{d_{i}-d_{0}} \phi_{i}^{b} \phi_{j}^{c} \phi_{i}^{d} \phi_{j}^{e},
\end{aligned}
$$

where $\mu, \nu$ are $d_{0}$-dimensional Lorentz indices, $a, b, c$ are color indices and $f^{a b c}$ are the structure constants of the gauge group. The vector field in $d_{i}$ dimensions is decomposed in a ( $d_{0}$-dimensional) vector $A_{\mu}^{a}$ and $d_{i}-d_{0}$ scalars $\phi_{i}^{a}$. The coupling of the $\phi^{4}$ interaction is given by

$$
\lambda=g^{2}
$$

and we call it $\lambda$ for reasons that will be clear in a moment. 
From (2.14), we can compute the amplitude with only external gluons ${ }^{5}$

$$
\begin{aligned}
\mathcal{A}_{\left(d_{i}, d\right)}^{(L)}= & \mathcal{A}_{\left(d_{0}, d\right)}^{(L)}+\sum_{m=0}^{L-1}\left(d_{i}-d_{0}-1\right)^{m} \\
& \times \sum_{n=1}^{L-m}\left(d_{i}-d_{0}\right)^{n} \mathcal{A}_{\left(d_{0}, d, n, m\right)}^{S(L)},
\end{aligned}
$$

where $A_{\left(d_{0}, d\right)}^{(L)}$ is the complete $L$-loop amplitude where all the internal legs are vectors and $\mathcal{A}_{\left(d_{0}, d, n, m\right)}^{S(L)}$ are specific combinations of diagrams with at least one scalar loop. Specifically, the diagrams contributing to $\mathcal{A}_{\left(d_{0}, d, n, m\right)}^{S(L)}$ are of order $\lambda^{m}$, i.e., they contain $m$ four-scalar interactions, and in addition have $n$ distinct purely scalar subdiagrams.

The coefficients for the scalar contributions in (2.16) can be understood as follows (see Fig. 1):

(1) The number of distinct flavors of scalars is $d_{i}-d_{0}$ and they all give the same contribution.

(2) Given a set of contiguous scalar propagators inside a diagram, when we draw the first scalar propagator, we need to multiply the diagram by a $d_{i}-d_{0}$ factor, corresponding to the distinct possible flavours.

(3) Inside the same set of contiguous scalar propagators, each vertex with two scalars and one vector must preserve the scalar flavor, while the four-scalar vertex changes it. Thus each power of $\lambda$ brings a $d_{i}-d_{0}-1$ factor.

(4) Every distinct set of scalar propagators leads to an additional $d_{i}-d_{0}$ factor.

(5) Since there are no external scalars, the number of distinct sets of scalar propagators plus the number of scalar quartic interactions coincides with the number of scalar loops:

$$
n+m=\text { \#scalar loops }
$$

(6) Clearly the number of scalar loops can be at most $L$. We can substitute (2.16) in (2.13) and, for simplicity, we choose

$$
d_{i}=d_{0}+i
$$

with $i=0, \ldots, L$. The final result should not depend on this choice, because the coefficient of a polynomial cannot depend on which point we choose for the fitting. After some manipulations, we find a closed expression which relates complete $L$-loop four-dimensional amplitudes to the same amplitudes in a higher integer dimension $d_{0}$ up to subtractions of scalar contribution:

\footnotetext{
${ }^{5}$ In this section, for the sake of clarity, we reserve the word vector only for the $d_{0}$-dimensional vector, whereas we refer to the four-dimensional equivalents as gluons.
}

$\mathcal{A}_{(4, d)}^{(L)}=\mathcal{A}_{\left(d_{0}, d\right)}^{(L)}+\sum_{m=0}^{L-1} \sum_{n=1}^{L-m}\left(4-d_{0}\right)^{n}\left(3-d_{0}\right)^{m} \mathcal{A}_{\left(d_{0}, d, n, m\right)}^{S(L)}$,

where, in order to prove this formula, we have used the identity

$$
\sum_{i=0}^{L} \frac{1}{\left(d_{i}-4\right)} \prod_{\substack{k=0 \\ k \neq i}}^{L} \frac{1}{\left(d_{k}-d_{i}\right)}=\prod_{j=0}^{L} \frac{1}{\left(d_{j}-4\right)} .
$$

The whole reasoning can be applied to a more generic scheme where $d_{s}=4$ (FHV scheme) is replaced by a generic $d_{s}$ (e.g., the HV scheme [61] with $d_{s}=4-2 \epsilon$ ). As long as we keep $d_{i}>d_{s}$ and $d_{i} \geq d$, all the previous steps are still applicable, and we arrive at

$$
\begin{aligned}
\mathcal{A}_{\left(d_{s}, d\right)}^{(L)}= & \mathcal{A}_{\left(d_{0}, d\right)}^{(L)}+\sum_{m=0}^{L-1} \sum_{n=1}^{L-m}\left(d_{s}-d_{0}\right)^{n}\left(d_{s}-d_{0}-1\right)^{m} \\
& \times \mathcal{A}_{\left(d_{0}, d, n, m\right)}^{S(L)},
\end{aligned}
$$

which at first sight is identical to (2.16). The nontrivial difference between the two expressions is that $d_{s}<d_{0}$, while we need $d_{i}>d_{0}(i=1, \ldots, L)$ in order to write (2.16). Moreover, as we stressed before, the quantity $\left(d_{i}-\right.$ $d_{0}$ ) has a precise physical meaning: it is the number of distinct flavors of scalars in the dimensional reduced theory (2.14). On the other hand, $\left(d_{s}-d_{0}\right)$ takes into account the number of extra spin d.o.f. in dimensional regularization ${ }^{6}$.

A posteriori, the fact that the two expressions are exactly the same is a consequence of our previous considerations. Indeed, one could have recognized the polynomial dependence of the amplitude on the dimensionality $d_{s}$ already from (2.16), and further identified (2.21) as its analytic continuation for $d_{s}-d_{0}<0$. Thus, starting from the dimensionally reduced Lagrangian (2.14), the dependence on the dimensionality $d_{s}$ emerges naturally, and the preceding considerations relating the $d_{i}$ to $d_{s}$ through the Vandermonde matrix may appear redundant. However, starting from the analysis of the dimensional dependence of the amplitudes provides a clear physical picture of the relation between $d_{s}, d$ and $d_{i}$.

Our expression reproduces the known results at one loop [12]

$$
\mathcal{A}_{\left(d_{s}, d\right)}^{(1)}=\mathcal{A}_{\left(d_{0}, d\right)}^{(1)}+\left(d_{s}-d_{0}\right) \mathcal{A}_{\left(d_{0}, d, 0,1\right)}^{S(1)},
$$

and two loops [18]

$\mathcal{A}_{\left(d_{s}, d\right)}^{(2)}=\mathcal{A}_{\left(d_{0}, d\right)}^{(2)}+\left(d_{s}-d_{0}\right) \Delta_{\left(d_{0}, d\right)}^{S}+\left(d_{s}-d_{0}\right)^{2} \Delta_{\left(d_{0}, d\right)}^{2 S}$,

\footnotetext{
${ }^{6}$ There is no dynamics in the dimensions $d_{i}-d_{0}$, while this could be not true for the dimensions $d_{0}-d_{s}$.
} 
where

$$
\Delta_{\left(d_{0}, d\right)}^{S}=\mathcal{A}_{\left(d_{0}, d, 0,1\right)}^{S(2)}-\mathcal{A}_{\left(d_{0}, d, 1,1\right)}^{S(2)}, \quad \Delta_{\left(d_{0}, d\right)}^{2 S}=\mathcal{A}_{\left(d_{0}, d, 0,2\right)}^{S(2)}+\mathcal{A}_{\left(d_{0}, d, 1,1\right)}^{S(2)} .
$$

Considering the two-loop expression in more detail, one sees that in [18] the four-scalar vertex is interpreted in terms of three fictitious flavor contributions:<smiles>CC(C)(C)C</smiles>

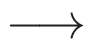

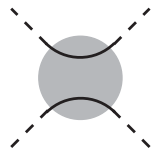

The two continuous lines in the grey blob represent the color flow inside the vertex. Considering Fig. 2 we see that, in our interpretation, the only diagram which at two loops involves this vertex contributes with a factor $\left(d_{s}-d_{0}\right)\left(d_{s}-d_{0}-1\right)$. However, splitting the vertex according to colour flow as above, the contribution of the same diagram can be attributed to terms containing a factor $\left(d_{s}-d_{0}\right)^{2}$ as well as $\left(d_{s}-d_{0}\right)$. Taking into account this different interpretation of the four-scalar vertex, the two methods perfectly match.

We emphasize that individually each $\mathcal{A}_{\left(d_{0}, d, n, m\right)}^{S(L)}$ is a gauge-invariant quantity: indeed, we know that $\mathcal{A}_{\left(d_{0}, d\right)}^{(L)}$ is gauge invariant and the same is true for $\mathcal{A}_{(4, d)}^{(L)}$, regardless of the choice of $d_{0}$. Since the coefficients of the scalar contributions depend on $d_{0}$, the single $\mathcal{A}_{\left(d_{0}, d, n, m\right)}^{S(L)}$ must be gauge invariant by themselves.

As in the case of the one-loop procedure, (2.19) can be applied also to form factors, as far as we bear in mind that more scalar operators are involved in higher-loop calculations, in addition to those entering already at one loop. These additional terms emerge clearly from (2.14). Indeed, for the operator $\operatorname{Tr} F^{2}$, beyond one-loop calculations we also need to subtract the scalar contribution from the $\phi^{4}$ operator:
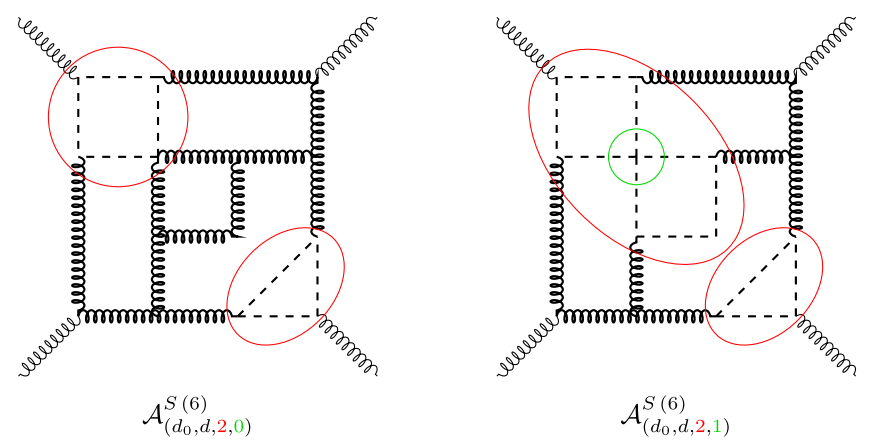

FIG. 1. Two of the many possible diagrams contributing to the scalar amplitudes at six loops. On the left-hand side an example contribution to $\mathcal{A}_{\left(d_{0}, d, 2,0\right)}^{S(6)}$ is shown. On the right-hand side the same diagram but with one of the gluon loops involving a fourpoint interaction replaced by a scalar. The latter diagram contributes to $\mathcal{A}_{\left(d_{0}, d, 2,1\right)}^{S(6)}$.

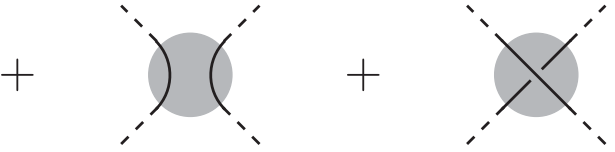

$$
F_{\mu \nu}^{a} F^{a \mu \nu} \mapsto g^{2} f^{a b d} f^{a c d} \phi^{b} \tilde{\phi}^{c} \phi^{d} \tilde{\phi}^{e},
$$

where $\phi$ and $\tilde{\phi}$ have to be scalars with different flavor. Its contribution has to be carefully taken into account in the subtraction with the right $d_{s}$-dependence. In particular, in the form factor equivalent of (2.16), its insertion brings a $\left(d_{i}-d_{0}\right)\left(d_{i}-d_{0}-1\right)$ coefficient, because of the flavor changing.

An equivalent reasoning is also valid for higherdimensional operators. For example, from the dimensional reduction procedure of the $\operatorname{Tr} F^{3}$ operator, we find that the additional scalar operators entering higher-loop calculations are

$f^{a b c} F_{\nu}^{a \mu} F_{\rho}^{b \nu} F_{\mu}^{c \rho} \mapsto\left\{\begin{array}{l}g f^{a b c} f^{a d e} D_{\mu} \phi^{b} D^{\mu} \tilde{\phi}^{c} \phi^{d} \tilde{\phi}^{e} \\ g^{3} f^{a b c} f^{a d e} f^{b f g} f^{c h i} \phi^{d} \tilde{\phi}^{e} \phi^{f} \hat{\phi}^{g} \tilde{\phi}^{h} \hat{\phi}^{i}\end{array}\right.$,

where the former enters the calculation at two-loop level, while the latter from three loops. We stress that $\phi, \tilde{\phi}$ and $\hat{\phi}$ represent three different scalar flavors. Then, in the generalization of (2.16) to form factors, the insertion of the scalar operators bring a factor of $\left(d_{i}-d_{0}\right)\left(d_{i}-d_{0}-1\right)$ and $\left(d_{i}-d_{0}\right)\left(d_{i}-d_{0}-1\right)\left(d_{i}-d_{0}-2\right)$ respectively. Following
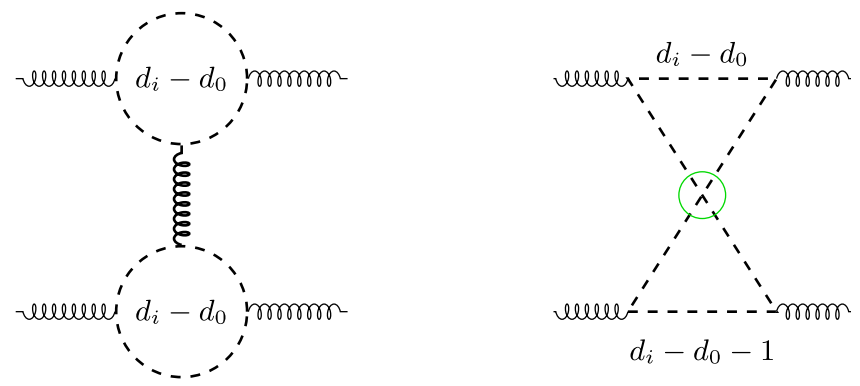

FIG. 2. Two two-loop diagrams for comparison. In the first case there are two disconnected scalar loops, and every loop admits $d_{i}-d_{0}$ different flavors leading to an overall factor $\left(d_{i}-d_{0}\right)^{2}$. The second diagram represents two scalar loops connected by a flavor-changing four-scalar vertex (highlighted in green). In this case there are $d_{i}-d_{0}$ allowed flavors in one loop but only $d_{i}-d_{0}-1$ in the second loop, which leads to an overall factor $\left(d_{i}-d_{0}\right)\left(d_{i}-d_{0}-1\right)$. 
the same procedure one can recover the scalar operators for $\operatorname{Tr} F^{4}$, which we do not write explicitly.

In the following we are going to apply this technique to one-loop calculations for form factors. We will always choose $d_{0}=6$, due to the existence of a powerful spinor helicity formalism in six dimensions [14,17].

A technical comment is in order here. In performing loop calculations, initially we treat the loop momenta as living in $d_{0}=6$ dimensions, instead of $d$. This procedure is well defined at the integrand level. Indeed, we know the functional dependence of the integrand on the $d-4$ components of the loop momenta, which appear only through rational combinations of $l_{i}^{(-2 \epsilon)} \cdot l_{j}^{(-2 \epsilon)}$ and $\mu_{i}^{2}$. Then, once we identify these combinations, we can treat the loop momenta as being $d$-dimensional and integrate over them ${ }^{7}$.

\section{TREE-LEVEL FORM FACTORS}

In this section we will provide all the analytic expressions of the tree-level color-ordered form factors required for loop calculations.

The tensorial structure of the field strength in four dimensions is given by the antisymmetric product of two vector representations

$$
\left(\frac{1}{2}, \frac{1}{2}\right) \wedge\left(\frac{1}{2}, \frac{1}{2}\right)=(1,0) \oplus(0,1),
$$

where we can choose each component to correspond to the helicity configurations \pm 1 . We then define the self-dual component of the free field strength as ${ }^{8}$

$$
F_{\mathrm{SD}, \alpha \dot{\alpha} \beta \dot{\beta}}:=\lambda_{\alpha} \lambda_{\beta} \epsilon_{\dot{\alpha} \dot{\beta}},
$$

which has helicity -1 and transforms in the $(1,0)$ representation of the Lorentz group. ${ }^{9}$ Then, the antiself-dual component, transforming in the $(0,1)$ representation is

$$
F_{\mathrm{ASD}, \alpha \dot{\alpha} \beta \dot{\beta}}=\epsilon_{\alpha \beta} \tilde{\lambda}_{\dot{\alpha}} \tilde{\lambda}_{\dot{\beta}} .
$$

In terms of $\mathrm{SU}^{*}(4)$ representations, the six-dimensional free field strength transforms in the $\mathbf{6} \wedge \mathbf{6}=\mathbf{1 5}$, which is the traceless part of $\mathbf{4} \otimes \overline{\mathbf{4}}$. Thus it can be written as [62]

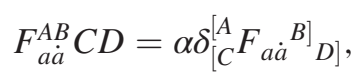

\footnotetext{
${ }^{7}$ It is worth mentioning that in terms of the six-dimensional spinor components the quantity mentioned above reads as follows: $l_{i}^{(-2 \epsilon)} \cdot l_{j}^{(-2 \epsilon)}=\frac{1}{2}\left(m_{i} \tilde{m}_{j}+m_{j} \tilde{m}_{i}\right)$ and $\mu_{i}^{2}=m_{i} \tilde{m}_{i}$.

${ }^{8}$ To clarify the abuse of nomenclature, the quantity it is the field strength in momentum space corresponding to a polarization vector of given helicity.

${ }^{9} \mathrm{We}$ could have used as definition the following: $F_{\mathrm{SD}, \alpha \dot{\alpha} \beta \dot{\beta}}:=p_{\alpha \dot{\alpha}} \varepsilon_{\beta \dot{\beta}}^{-}-p_{\beta \dot{\beta}} \varepsilon_{\alpha \dot{\alpha}}^{-}=-\sqrt{2} \lambda_{\alpha} \lambda_{\beta} \epsilon_{\dot{\alpha} \dot{\beta}}$. As we can see the only difference is an overall $-\sqrt{2}$ factor.
}

where $\alpha$ is a numerical coefficient to be fixed and $F_{a \dot{a}}{ }^{A}{ }_{B}$ is such that $F_{a \dot{a}}{ }_{A}{ }_{A}=0 .{ }^{10}$ In spinor helicity variables this quantity is [62]

$$
F_{a \dot{a}}{ }^{A} B=\lambda_{a}^{A} \tilde{\lambda}_{\dot{a} B}
$$

which is indeed traceless thanks to the six-dimensional Dirac equation (B10). Upon dimensionally reducing (3.5) down to four dimensions we match it with (3.2) and (3.3), which fixes the proportionality coefficient to be $\alpha=2$.

\section{A. $\operatorname{Tr} F^{2}$ form factors}

In this section we consider the operator

$$
\mathcal{O}_{2}:=F_{\mu \nu}^{a} F^{a \mu \nu} .
$$

In four dimensions $\mathcal{O}_{2}$ splits naturally into the sum of the traces of the self-dual and the antiself-dual components of the field strength:

$$
\operatorname{Tr} F^{2}=\operatorname{Tr} F_{\mathrm{SD}}^{2}+\operatorname{Tr} F_{\mathrm{ASD}}^{2}
$$

It is trivial to identify these two four-dimensional components of the color-ordered form factor:

$$
\begin{aligned}
F_{\mathcal{O}_{2}}^{(0)}\left(1^{+}, 2^{+} ; q\right) & =2[12][21], \\
F_{\mathcal{O}_{2}}^{(0)}\left(1^{-}, 2^{-} ; q\right) & =2\langle 12\rangle\langle 21\rangle .
\end{aligned}
$$

On the other hand, the six-dimensional form factor is

$$
F_{\mathcal{O}_{2}}^{(0)}\left(1_{a \dot{a}}, 2_{b \dot{b}} ; q\right)=2\left\langle 1_{a} 2_{\dot{b}}\right]\left\langle 2_{b} 1_{\dot{a}}\right]
$$

where the definitions of the spinor brackets, in both four and six dimensions, and the conventions adopted can be found in Appendices A and B. Using the particular embedding of the four-dimensional into the six-dimensional space introduced in Appendix B 3 we find that ${ }^{11}$

$$
\begin{aligned}
& \left.F_{\mathcal{O}_{2}}^{(0)}\left(1_{1 \mathrm{i}}, 2_{1 \mathrm{i}} ; q\right)\right|_{4 \mathrm{D}}=F_{\mathcal{O}_{2}}^{(0)}\left(1^{+}, 2^{+} ; q\right), \\
& \left.F_{\mathcal{O}_{2}}^{(0)}\left(1_{2 \dot{2}}, 2_{2 \dot{2}} ; q\right)\right|_{4 \mathrm{D}}=F_{\mathcal{O}_{2}}^{(0)}\left(1^{-}, 2^{-} ; q\right) .
\end{aligned}
$$

An analogous statement is true also for amplitudes, where all the four-dimensional helicity configurations can be recovered from the six-dimensional amplitude. ${ }^{12}$

\footnotetext{
${ }^{10} A, B, \ldots=1, \ldots, 4$ are indices in the (anti)fundamental representation of $\mathrm{SU}^{*}(4)$ and $a, \dot{a}$ are indices of the six-dimensional little group (for a detailed discussion see Appendix B).

${ }^{11}$ Four-dimensional limit here means choosing appropriate little-group indices corresponding to the desired helicity configuration in four dimensions, and the taking $m_{i}, \tilde{m}_{i} \rightarrow 0$ for any particle $i$.

${ }^{12}$ Further details on the relation between four and six-dimensional tree-level quantities can be found in Appendix C.
} 
The scalar form factor is obtained from (2.6), and we find

$$
F_{\mathcal{O}_{2, s}}^{(0)}(1,2 ; q)=-\left\langle 1_{a}, 2_{\dot{b}}\right]\left\langle 1^{a}, 2^{\dot{b}}\right]=2 s_{12},
$$

where

$$
\mathcal{O}_{2, s} \propto(D \phi)^{2}:=D_{\mu} \phi^{a} D^{\mu} \phi^{a}
$$

The normalization of (3.11) has been fixed by matching the four-dimensional limit of this operator with that of the scalar components of (3.9), which must yield the same result. Of course, if one starts from the Lagrangian (2.6) and computes the minimal form factors of the two operators on the right-hand side, the resulting relative normalization would be the same. The four-dimensional matching prescription is much faster for more complex operators. Let us stress that it would not be possible to implement the scalar subtraction just by excluding the little group components that in four dimensions behave like scalars. Indeed, this would bring us to a result which is not invariant under a little group transformation of the internal six-dimensional legs. In particular, for the subtraction we need a quantity that behaves as a scalar in six dimensions and matches the scalar components of the dimensional-reduced gluon in four dimensions, as shown in Appendix C.

Using BFCW recursion relation $[43,63]$ in six dimensions [17] we have derived the six-dimensional nonminimal form factors with three external legs at tree level, both for the gluon and the scalar operators. The results for $\mathcal{O}_{2}$ with three gluons reads

$$
\begin{aligned}
& F_{\mathcal{O}_{2}}^{(0)}\left(1_{a \dot{a}}, 2_{b \dot{b}}, 3_{c \dot{c}} ; q\right) \\
& =\frac{2}{s_{23} s_{31}}\left\langle 1_{a} 2_{\dot{b}}\right]\left\langle 2_{b} 1_{\dot{a}}\right]\left\langle 3_{c}\left|\not p_{1} \not \phi_{2}\right| 3_{\dot{c}}\right]+\text { cyclic } \\
& \quad+2\left(\frac{1}{s_{12}}+\frac{1}{s_{23}}+\frac{1}{s_{31}}\right)\left(\left\langle 1_{a} 2_{\dot{b}}\right]\left\langle 2_{b} 3_{\dot{c}}\right]\left\langle 3_{c} 1_{\dot{a}}\right]\right. \\
& \left.\quad-\left[1_{\dot{a}} 2_{b}\right\rangle\left[2_{\dot{b}} 3_{c}\right\rangle\left[3_{\dot{c}} 1_{a}\right\rangle\right),
\end{aligned}
$$

which agrees with the analogous result computed from Feynman diagrams in [16], upon some algebraic manipulation. As a further consistency check we verified that in the four-dimensional limit the different helicity components match the results of [33].

Furthermore, in the scalar subtraction we need to take into account an additional contribution, namely the form factor of the operator $\mathcal{O}_{2}$ with two external scalars and one gluon, which is different from zero. Indeed, this is given by:

$$
F_{\mathcal{O}_{2}}^{(0)}\left(1,2,3_{c \dot{c}} ; q\right)=-\frac{2}{s_{12}}\left\langle 3_{c}\left|\not \not_{1} \not \not_{2}\right| 3_{\dot{c}}\right] .
$$

Finally, the nonminimal scalar form factor of $\mathcal{O}_{2, s}$ can be shown to be

$$
F_{\mathcal{O}_{2, s}}^{(0)}\left(1,2,3_{c \dot{c}} ; q\right)=-\frac{2 q^{2}}{s_{23} s_{31}}\left\langle 3_{c}\left|\not p_{1} \not \not_{2}\right| 3_{\dot{c}}\right] .
$$

For a detailed derivation of (3.13)-(3.15) see Appendix D. The sum of (3.14) and (3.15) agrees with the result of [16].

\section{B. $\operatorname{Tr} F^{3}$ form factors}

Consider now the operator

$$
\mathcal{O}_{3}:=\operatorname{Tr} F^{\mu}{ }_{\nu} F_{\rho}^{\nu} F^{\rho}{ }_{\mu}
$$

Similarly to the case of $\operatorname{Tr} F^{2}$, this operator splits, in four dimensions, into a self-dual and antiself-dual part

$$
\mathcal{O}_{3}:=\operatorname{Tr} F^{3}=\operatorname{Tr} F_{\mathrm{SD}}^{3}+\operatorname{Tr} F_{\mathrm{ASD}}^{3} .
$$

Consequently, the only possible helicity configurations of the minimal tree-level form factors are all-plus and allminus:

$$
\begin{gathered}
F_{\mathcal{O}_{3}}^{(0)}\left(1^{+}, 2^{+}, 3^{+} ; q\right)=-2[12][23][31], \\
F_{\mathcal{O}_{3}}^{(0)}\left(1^{-}, 2^{-}, 3^{-} ; q\right)=2\langle 12\rangle\langle 23\rangle\langle 31\rangle .
\end{gathered}
$$

In six dimensions the minimal form factor is given by

$$
\begin{aligned}
F_{\mathcal{O}_{3}}^{(0)}\left(1_{a \dot{a}}, 2_{b \dot{b}}, 3_{c \dot{c}}\right)= & F_{1 a \dot{a} C D}^{A B} F_{2 b \dot{b} E F}^{C D} F_{3 c \dot{c} A B}^{E F} \\
= & -\left\langle 1_{a} 2_{\dot{b}}\right]\left\langle 2_{b} 3_{\dot{c}}\right]\left\langle 3_{c} 1_{\dot{a}}\right] \\
& +\left[1_{\dot{a}} 2_{b}\right\rangle\left[2_{\dot{b}} 3_{c}\right\rangle\left[3_{\dot{c}} 1_{a}\right\rangle,
\end{aligned}
$$

where $F_{a \dot{a} C D}^{A B}$ is defined in (3.4). We can obtain the corresponding scalar operator from (2.9), which states that

$$
\mathcal{O}_{3, s} \propto \operatorname{Tr}(D \phi)^{2} F:=\operatorname{Tr} D_{\mu} \phi D_{\nu} \phi F^{\mu \nu}
$$

Thus

$F_{\mathcal{O}_{3, s}}^{(0)}\left(1,2,3_{c \dot{c}}\right):=\frac{1}{2} p_{1}^{A B} p_{2 C D} F_{3 c \dot{c} A B}^{C D}=\left\langle 3_{c}\left|\not p_{1} \not \not_{2}\right| 3_{\dot{c}}\right]$,

where, once again, the normalization is fixed by matching the four-dimensional limits of this quantity with the scalar configuration of (3.19).

As a final remark, we point out that $\mathcal{O}_{3}$ is not the only mass-dimension six operator which appears in the YangMills theories (also with matter). One also has a contribution from

$$
\tilde{\mathcal{O}}_{3}:=D^{\alpha} F^{a \mu \nu} D_{\alpha} F_{\mu \nu}^{a}
$$

However, it is easy to see that the minimal form factor for $\tilde{\mathcal{O}}_{3}$ can be related to the one of $\mathcal{O}_{2}$ as 


$$
F_{\tilde{\mathcal{O}}_{3}}^{(0)}\left(1_{a \dot{a}}, 2_{b \dot{b}} ; q\right)=s_{12} F_{\mathcal{O}_{2}}^{(0)}\left(1_{a \dot{a}}, 2_{b \dot{b}} ; q\right) .
$$

Further Lorentz contractions of two covariant derivatives and two field strengths, such as $D^{\mu} F_{\mu}^{a \nu} D^{\rho} F_{\rho \nu}^{a}$, are ruled out or expressed in terms of the operators previously mentioned thanks to the equations of motion. In particular, in the case of pure Yang-Mills theory $\tilde{\mathcal{O}}_{3}$ can be expressed as a linear combination of $\mathcal{O}_{2}$ and $\mathcal{O}_{3}$ through the equations of motion. For a detailed discussion, see [64].

Finally, we provide the tree-level expressions needed for the one-loop computation of the nonminimal form factor of $\mathcal{O}_{3}$ which are:

(i) the nonminimal tree-level form factor of $\mathcal{O}_{3}$ with four gluons

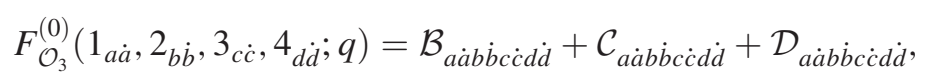

with

$$
\begin{aligned}
& \mathcal{B}_{a \dot{a} b \dot{b} \dot{c} \dot{c} d \dot{d}}=\left(-\left\langle 1_{a} 2_{\dot{b}}\right]\left\langle 2_{b} 3_{\dot{c}}\right]\left\langle 3_{c} 1_{\dot{a}}\right]+\left[1_{\dot{a}} 2_{b}\right\rangle\left[2_{\dot{b}} 3_{c}\right\rangle\left[3_{\dot{c}} 1_{a}\right\rangle\right) \frac{\left\langle 4_{d}\left|\not \phi_{1} \not{ }_{3}\right| 4_{\dot{d}}\right]}{s_{34} s_{41}}+\text { cyclic, } \\
& \mathcal{C}_{a \dot{a} b \dot{b} \dot{b} \dot{c} d \dot{d}}= \frac{\left\langle 1_{a} 2_{\dot{b}}\right]\left\langle 2_{b} 4_{\dot{d}}\right]\left\langle 4_{d} 3_{\dot{c}}\right]\left\langle 3_{c} 1_{\dot{a}}\right]+\left[1_{\dot{a}} 2_{b}\right\rangle\left[2_{\dot{b}} 4_{d}\right\rangle\left[4_{\dot{d}} 3_{c}\right\rangle\left[3_{\dot{c}} 1_{a}\right\rangle}{s_{34}}+\text { cyclic, } \\
& \mathcal{D}_{a \dot{a} b \dot{b} c \dot{c} d \dot{d}}=-\left(\sum_{i=1}^{4} \frac{1}{s_{i i+1}}\right)\left(\left\langle 1_{a} 2_{\dot{b}}\right]\left\langle 2_{b} 3_{\dot{c}}\right]\left\langle 3_{c} 4_{\dot{d}}\right]\left\langle 4_{d} 1_{\dot{a}}\right]+\left[1_{\dot{a}} 2_{b}\right\rangle\left[2_{\dot{b}} 3_{c}\right\rangle\left[3_{\dot{c}} 4_{d}\right\rangle\left[4_{\dot{d}} 1_{a}\right\rangle\right)
\end{aligned}
$$

(ii) the nonminimal tree-level form factor of $\mathcal{O}_{3}$ with two external scalars

$$
F_{\mathcal{O}_{3}}^{(0)}\left(1,2,3_{c \dot{c}}, 4_{d \dot{d}} ; q\right)=\frac{1}{s_{12}}\left(\left\langle 3_{c} 4_{\dot{d}}\right]\left\langle 4_{d}\left|\not \not_{1} \not \not_{2}\right| 3_{\dot{c}}\right]-\left\langle 4_{d} 3_{\dot{c}}\right]\left\langle 3_{c}\left|\not \not_{1} \not \not_{2}\right| 4_{\dot{d}}\right]\right)
$$

(iii) the nonminimal tree-level form factor of $\mathcal{O}_{3, s}$ with two external scalars

$$
\begin{aligned}
& F_{\mathcal{O}_{3, s}}^{(0)}\left(1,2,3_{\dot{1}}, 4_{d \dot{d}} ; q\right)=\frac{\left\langle 3_{c}\left|\not \not_{4} \not \not \not_{2}\right| 3_{\dot{c}}\right]\left\langle 4_{d}\left|\not \not_{1} \not \not_{2}\right| 4_{\dot{d}}\right]}{s_{23} s_{34}}+\frac{\left\langle 4_{d}\left|\not \not_{1} \not \not_{3}\right| 4_{\dot{d}}\right]\left\langle 3_{c}\left|\not \not_{1} \not \not_{2}\right| 3_{\dot{c}}\right]}{s_{34} s_{41}} \\
& +\left\langle 3_{c}\left|\not \not_{2} \not \not_{1}\right| 4_{\dot{d}}\right]\left\langle 4_{d} 3_{\dot{c}}\right]\left(\frac{1}{s_{34}}+\frac{1}{s_{23}}+\frac{1}{s_{41}}\right) \\
& -\left\langle 4_{d}\left|\not \not_{2} \not \not_{1}\right| 3_{\dot{c}}\right]\left\langle 3_{c} 4 \dot{d}\right] \frac{1}{s_{34}}+\left\langle 3_{c}\left|\not \not_{2}\right| 4_{d}\right\rangle\left[3_{\dot{c}}\left|\not \phi_{1}\right| 4_{\dot{d}}\right]\left(\frac{1}{s_{23}}+\frac{1}{s_{41}}\right) \text {. }
\end{aligned}
$$

These formulas have been obtained by requiring the sixdimensional form factor to match, upon taking the fourdimensional limit, the known four-dimensional expressions in different helicity configurations $[28,37,65,66]$. The resulting ansatz was then numerically compared with the results from Feynman diagrams and a complete match was found.

\section{C. $\operatorname{Tr} F^{4}$ and higher dimensional form factors}

The fourth power in the field strength can be considered as a turning point in the general behaviour of the operators, for reasons which will become clear in a moment. The first study of renormalization properties of gluonic operators of dimension up to eight was carried out in [67] and with more recent techniques in [68]. It turns out that we can have four possible independent operators involving different contractions of four field strengths:

$$
\begin{array}{ll}
\operatorname{Tr} F^{\mu}{ }_{\nu} F_{\rho}^{\nu} F^{\rho}{ }_{\sigma} F^{\sigma}{ }_{\mu}, & \operatorname{Tr} F^{\mu \nu} F_{\mu \nu} F^{\rho \sigma} F_{\rho \sigma}, \\
\operatorname{Tr} F^{\mu}{ }_{\nu} F_{\sigma}^{\rho} F^{\nu}{ }_{\rho} F^{\sigma}{ }_{\mu}, & \operatorname{Tr} F^{\mu \nu} F^{\rho \sigma} F_{\mu \nu} F_{\rho \sigma} .
\end{array}
$$

In pure gauge theories, which we are considering in this work, all these operators can appear with independent coefficients, while they are no more independent in the low energy effective action from the superstring theory [69-71]. In this section we will focus only on the first operator, which we will refer to as $\operatorname{Tr} F^{4}$ : 


$$
\mathcal{O}_{4}:=\operatorname{Tr} F^{4}:=\operatorname{Tr} F^{\mu}{ }_{\nu} F_{\rho}^{\nu} F^{\rho}{ }_{\sigma} F^{\sigma}{ }_{\mu} .
$$

This encloses all the main features of the operators with higher powers in the field strength, and at the end of this section we will be able to generalize some results to a peculiar operator involving a consecutive chain of $n$ field strengths.

In four dimensions the main difference between $\operatorname{Tr} F^{4}$ and the lower-power cases is that the structure of this operator allows the mixing of the self- and antiself-dual components, i.e., schematically

$$
\operatorname{Tr} F^{4} \simeq \operatorname{Tr} F_{\mathrm{SD}}^{4}+\operatorname{Tr}\left(F_{\mathrm{SD}}^{2} F_{\mathrm{ASD}}^{2}\right)+\operatorname{Tr} F_{\mathrm{ASD}}^{4} .
$$

Thus the usual all-plus (all-minus) minimal form factors appear along with MHV-like quantities:

$$
\begin{aligned}
& F_{\mathcal{O}_{4}}^{(0)}\left(1^{+}, 2^{+}, 3^{+}, 4^{+} ; q\right)=2[12][23][34][41], \\
& F_{\mathcal{O}_{4}}^{(0)}\left(1^{+}, 2^{+}, 3^{-}, 4^{-} ; q\right)=[12]^{2}\langle 34\rangle^{2}, \\
& F_{\mathcal{O}_{4}}^{(0)}\left(1^{+}, 2^{-}, 3^{+}, 4^{-} ; q\right)=[13]^{2}\langle 24\rangle^{2},
\end{aligned}
$$

and all the other configurations can be obtained by symmetry and parity arguments.

In six dimensions the minimal form factor is

$$
\begin{aligned}
F_{\mathcal{O}_{4}}^{(0)}\left(1_{a \dot{a}}, 2_{b \dot{b}}, 3_{c \dot{c}}, 4_{\dot{d} \dot{d}} ; q\right)= & F_{1 a \dot{a} C D}^{A B} F_{2 b \dot{b} E F}^{C D} F_{3 c \dot{c} G H}^{E F} F_{4 \dot{d} \dot{d} A B}^{G H} \\
\stackrel{(\mathrm{B} .14)}{=} & \left\langle 1_{a} 2_{\dot{b}}\right]\left\langle 2_{b} 3_{\dot{c}}\right]\left\langle 3_{c} 4_{\dot{d}}\right]\left\langle 4_{d} 1_{\dot{a}}\right] \\
& +\left[1_{\dot{a}} 2_{b}\right\rangle\left[2_{\dot{b}} 3_{c}\right\rangle\left[3_{\dot{c}} 4_{d}\right\rangle\left[4_{\dot{d}} 1_{a}\right\rangle \\
& +\left\langle 1_{a} 2_{b} 3_{c} 4_{d}\right\rangle\left[1_{\dot{a}} 2_{\dot{b}} 3_{\dot{c}} 4_{\dot{d}}\right],
\end{aligned}
$$

where we notice that at this power of the field strength the new structure $\langle\cdots \cdot\rangle[\cdots \cdot \cdot$ involving four-spinor invariants appears, which is very reminiscent of the four-point amplitude. This new structure gives us the MHV-like components in (3.31) when we consider the appropriate little-group configurations in the four-dimensional limit (see Appendix C).

We have already identified the scalar operator associated to $\operatorname{Tr} F^{4}$ in (2.10) and we define

$$
\mathcal{O}_{4, s} \propto \operatorname{Tr} D_{\mu} \phi D_{\nu} \phi F^{\nu}{ }_{\rho} F^{\rho \mu}
$$

such that its minimal form factor is

$$
\begin{aligned}
F_{\mathcal{O}_{4, s}\left(1,2,3_{c \dot{c}}, 4_{d \dot{d}} ; q\right)=} & \frac{1}{2} p_{1}^{A B} p_{2 C D} F_{3 c \dot{c} E F}^{C D} F_{4 \dot{d} \dot{d} A B}^{E F} \\
= & -\left\langle 3_{c}\left|\not \not_{2} \not \not_{1}\right| 4_{\dot{d}}\right]\left\langle 4_{d} 3_{\dot{c}}\right] \\
& +\frac{1}{4}\left\langle 2^{a} 2_{a} 3_{c} 4_{d}\right\rangle\left[1_{\dot{a}} 1^{\dot{a}} 3_{\dot{c}} 4_{\dot{d}}\right]
\end{aligned}
$$

The expression of $\operatorname{Tr} F^{4}$ gives us some insight about the operators involving the $n$th power of the field strength, where the Lorentz indices are contracted between adjacent field strengths, which we will refer to as $\operatorname{Tr} F^{n}$ :

$$
\mathcal{O}_{n}:=\operatorname{Tr} F^{n}=\operatorname{Tr} F_{\mu_{1}}^{\mu_{2}} F_{\mu_{2}}^{\mu_{3}} \cdots F_{\mu_{n-1}}^{\mu_{n}} F_{\mu_{n}}^{\mu_{1}} .
$$

It is easy to show that this operator can be decomposed in a sum of double traces (in the Lorentz indices) on the selfdual and antiself-dual parts, schematically ${ }^{13}$ :

$$
\operatorname{Tr} F^{n} \simeq \sum_{i=0}^{n} \operatorname{Tr}\left(F_{\mathrm{SD}}^{n-i} F_{\mathrm{ASD}}^{i}\right)
$$

Take two disjoint and ordered subsets of labels $S_{+}=$ $\left\{p_{k}\right\}_{k=1 \ldots i}$ and $S_{-}=\left\{q_{k}\right\}_{k=1 \ldots n-i}$, with $S_{+} \cup S_{-}=$ $\{1, \ldots, n\}$. Then all tree level form factors, for any helicity configuration, can be written in a very compact way:

$F_{\mathcal{O}_{n}}^{(0)}\left(1^{h_{1}}, \ldots, n^{h_{n}} ; q\right)=c_{n, i} \prod_{k=1}^{i}\left[p_{k} p_{k+1}\right] \prod_{k=i+1}^{n}\left\langle q_{k} q_{k+1}\right\rangle$,

where the overall coefficient is

$$
c_{n, i}= \begin{cases}2 & i=0 \\ (-1)^{n-i} & i \neq 0, n . \\ (-)^{n} 2 & i=n\end{cases}
$$

An explicit example of this general formula is given by

$F_{\mathcal{O}_{5}}^{(0)}\left(1^{-}, 2^{+}, 3^{-}, 4^{-}, 5^{+} ; q\right)=-\langle 13\rangle\langle 34\rangle\langle 41\rangle[25][52]$.

The structure of $\operatorname{Tr} F^{n}$ form factors in six dimensions is much more complicated than the four-dimensional one, the number of terms grows very fast, but nonetheless some general pattern can be observed. In particular if we restrict to a kinematic configuration for which only some of the legs are truly six dimensional and the others are defined on the embedded four-dimensional subspace, the formulas are much easier and compact. In principle, this is all we need in order to calculate rational terms with the dimensional reconstruction technique, since we need to consider only the limited number of internal loop legs as six dimensional. As an example, consider the minimal form factor of $\operatorname{Tr} F^{n}$ with two six-dimensional legs and $n-2$ four-dimensional legs in the all-plus helicity configuration. The general expression is given by

\footnotetext{
${ }^{13}$ We stress that this general structure was hidden by lower power-operators because the field strength is traceless: $\operatorname{Tr} F_{S D}^{n-1} F_{A S D}=\operatorname{Tr} F_{S D} F_{A S D}^{n-1}=0$.
} 


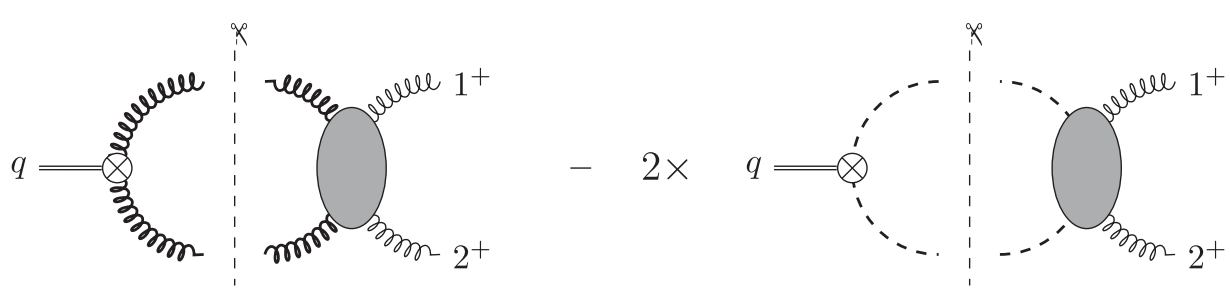

FIG. 3. Two-particle cut of the one-loop form factor $\operatorname{Tr} F^{2}$ in six dimensions.

$$
\begin{aligned}
\operatorname{Tr} F^{n}\left(1_{a \dot{a}}, 2_{b \dot{b}}, 3^{+}, \ldots, n^{+}\right)= & \left(\left\langle 1_{a} 2_{\dot{b}}\right]\left\langle 2_{b} 3_{\mathrm{i}}\right][34]\left\langle n_{1} 1_{\dot{a}}\right]\right. \\
& +\left[1_{\dot{a}} 2_{b}\right\rangle\left[2_{\dot{b}} 3_{1}\right\rangle[34]\left[n_{\mathrm{i}} 1_{a}\right\rangle \\
& \left.+\left\langle 1_{a} 2_{b} n_{1} 3_{1}\right\rangle\left[1_{\dot{a}} 2_{\dot{b}} 3_{\mathrm{i}} 4_{\mathrm{i}}\right]\right) \\
& \times \prod_{i=4}^{n-1}[i i+1] .
\end{aligned}
$$

This result can be found by observing that the combination $\lambda_{i a}^{A} \tilde{\lambda}_{i B \dot{a}}$ appears only once for each six-dimensional leg, which allows to write an ansatz comprising every possible combination with arbitrary coefficients to be fixed. The coefficients can then be determined by taking the fourdimensional limit of the six-dimensional gluons and requiring the form factor to match (3.37). For the sake of comparison, if we take $n=6$ the three terms of (3.40) come from a fully six-dimensional expression of 39 terms which has already been reduced from initial 52 terms using Schouten identity.

\section{ONE-LOOP FORM FACTORS}

In this section we will consider a number of one-loop applications of the dimensional reconstruction procedure discussed so far. The results obtained for the minimal form factors of $\operatorname{Tr} F^{2}$ and $\operatorname{Tr} F^{3}$ were already known in the literature. We prove that the latter has no rational terms, as it has also been argued by [28]. These calculations will be useful to set the stage and give an example of the procedure before dealing with more involved operators and kinematic configurations. In particular, we reproduce the known nonminimal form factor of $\operatorname{Tr} F^{2}$ with three positive-helicity external gluons. Finally, we compute the complete minimal form factor of $\operatorname{Tr} F^{n}$ with $n=4$ at one loop and generalize some of the results to arbitrary $n$.

\section{A. The minimal $\operatorname{Tr} F^{2}$ form factors}

As a first proof of concept of the method we will confirm the well known statement that the minimal form factor of the operator $\operatorname{Tr} F^{2}$ in pure Yang-Mills does not have any rational terms. In particular, we will consider the all-plus helicity configuration.

The quantity we want to compute can be written as

$$
\begin{array}{r}
F_{\mathcal{O}_{2}}^{(1)}\left(1^{+}, 2^{+} ; q\right):=F_{\mathcal{O}_{2}}^{(0)}\left(1^{+}, 2^{+} ; q\right) \cdot f^{(2)}\left(s_{12}\right) \\
=2[12][21] \cdot f^{(2)}\left(s_{12}\right),
\end{array}
$$

where we factored out all the helicity dependence in the tree-level prefactor, and $f^{(2)}\left(s_{12}\right)$ is a function only of the Mandelstam variable $s_{12}$. As explained in Sec. II this quantity can be computed using (2.7):

$$
f^{(2)}\left(s_{12}\right)=f_{6 \mathrm{D}}^{(2)}\left(s_{12}\right)-2 f_{\phi}^{(2)}\left(s_{12}\right),
$$

where $f_{6 \mathrm{D}}^{2}\left(s_{12}\right)$ and $f_{\phi}^{2}\left(s_{12}\right)$ are the form factors with sixdimensional internal gluons or scalars respectively, normalized by the corresponding tree-level quantity.

At one loop, the two-particle cut represented in Fig. 3 is ${ }^{14}$

$$
\begin{aligned}
\left.f_{6 \mathrm{D}}^{(2)}\left(s_{12}\right)\right|_{2-\mathrm{cut}}= & \frac{1}{2[12][21]} \int \mathrm{d} \operatorname{LIPS} F_{\mathcal{O}_{2}}^{(0)}\left(-l_{1}^{a \dot{a}},-l_{2}^{b \dot{b}}\right) \\
& \times \mathcal{A}_{g}^{(0)}\left(l_{2 b \dot{b}}, l_{1 a \dot{a}}, 1_{1 \dot{1}}, 2_{2 \dot{2}}\right) .
\end{aligned}
$$

In order to simplify this expression we decompose the sixdimensional quantities in terms of four-dimensional ones, as explained in detail in Section B 3. These calculations are rather lengthy and we have devised a Mathematica package to deal with them (see Appendix F for a detailed presentation). In general, we write six-dimensional expressions in terms of $\left\{\lambda_{i \alpha}, \tilde{\lambda}_{i \dot{\alpha}}, \mu_{i \alpha}, \tilde{\mu}_{i \dot{\alpha}}, m_{i}, \tilde{m}_{i}\right\}$ with $i=1,2, l_{1}$, $l_{2}$, as explained in Appendix B 3. Imposing that the external legs are defined in four dimensions is equivalent to setting $m_{j}=0$ and $\tilde{m}_{j}=0$ for $j=1,2$, which automatically removes any dependence of $f^{(2)}$ on $\mu_{j \alpha}$ and $\tilde{\mu}_{j \dot{\alpha}}$. From (B22), momentum conservation implies

$$
\sum_{i} m_{i}=0, \quad \sum_{i} \tilde{m}_{i}=0
$$

Only the two internal legs $l_{1}$ and $l_{2}$ have to be kept in six dimensions, in other words $p_{i}^{5}, p_{i}^{6} \neq 0$ for $i=l_{1}, l_{2}$, which implies

$$
m_{l_{2}}=-m_{l_{1}}:=-m, \quad \tilde{m}_{l_{2}}=-\tilde{m}_{l_{1}}:=-\tilde{m},
$$

where

$$
\mu^{2}=m \tilde{m},
$$

with $\mu^{2}$ defined in (2.3). The result for the complete integrand in (4.3) is, schematically,

\footnotetext{
${ }^{14}$ The explicit expression of $\mathcal{A}_{g}$ can be found in Appendix C.
} 


$$
\begin{aligned}
\mathcal{I}= & \frac{i\left\langle l_{1} l_{2}\right\rangle^{2}\left[l_{2} l_{1}\right]^{2}}{s_{12} s_{2 l_{2}}}+\mu^{2}(4 \text { terms })+\mu^{4}(17 \text { terms }) \\
& +\mu^{6}(5 \text { terms })+\mu^{8}(1 \text { term })
\end{aligned}
$$

where the Mandelstam invariants are defined in terms of sixdimensional momenta. It is important to note that the dependence on $\mu_{i}$ and $\tilde{\mu}_{i}$ is spurious and we can choose these "reference momenta" in order to cancel as many terms as possible from our result. After doing so one has to be careful in identifying the loop momenta and Mandelstam invariants consistently with this choice. A particularly convenient choice is

$$
\mu_{l_{1}} \rightarrow \lambda_{l_{2}}, \quad \mu_{l_{2}} \rightarrow \lambda_{l_{1}}, \quad \tilde{\mu}_{l_{2}} \rightarrow \tilde{\mu}_{l_{1}} .
$$

Doing so, we immediately arrive at

$$
\left.f_{6 \mathrm{D}}^{(2)}\left(s_{12}\right)\right|_{2-\mathrm{cut}}=\int \mathrm{d} \operatorname{LIPS}\left(-i \frac{s_{12}}{s_{2 l_{2}}}+2 i \frac{\mu^{2}}{s_{2 l_{2}}}\right) .
$$

Next we repeat a similar computation for the two-particle cut with internal gluons replaced by scalars:

$$
\begin{aligned}
\left.f_{\phi}^{(2)}\left(s_{12}\right)\right|_{2-\text { cut }}= & \frac{1}{2[12][21]} \int \operatorname{dLIPS} F_{\mathcal{O}_{2, s}}^{(0)}\left(-l_{1},-l_{2}\right) \\
& \times \mathcal{A}^{(0)}\left(l_{2}, l_{1}, 1_{a \dot{a}}, 2_{b \dot{b}}\right) \\
= & \int \mathrm{dLIPS} i \frac{\mu^{2}}{s_{2 l_{2}}} .
\end{aligned}
$$

Taking the difference between (4.9) and twice (4.10) leads to the desired four-dimensional result

$$
\left.f^{(2)}\left(s_{12}\right)\right|_{2-\text { cut }}=-i s_{12} \int \mathrm{dLIPS} \frac{1}{s_{2 l_{2}}} .
$$

It is important to stress that in order to perform the scalar subtraction consistently, one needs first to write both $f_{6 D}^{(2)}$ and $f_{\phi}^{(2)}$ as functions of the full $d$-dimensional momenta and Mandelstam invariants, in order to eliminate any dependence on the choice of the arbitrary helicity spinors $\mu_{i}$ and $\tilde{\mu}_{i}$. We can directly read off the one-loop result from (4.11):

$$
f^{(2)}\left(s_{12}\right)=-i s_{12} \cdot q=
$$

where the triangle integral with outgoing momenta $\left(p_{1}, p_{2}, q\right)$ is defined in Appendix E.

As anticipated, our result (4.12) does not contain any $\mu^{2}$ term i.e., any rational term, and is thus in agreement with the very well known result. An equivalent result holds for the all-minus helicity configuration. As expected, there are no bubbles in the result, because both $\operatorname{Tr} F_{\mathrm{SD}}^{2}$ and $\operatorname{Tr} F_{\mathrm{ASD}}^{2}$ are protected operators.

\section{B. The nonminimal $\operatorname{Tr} F^{2}$ form factor}

In this section we address the computation of the oneloop nonminimal form factor of the operator $\operatorname{Tr} F^{2}$. As usual we begin by defining the normalized quantity $f^{(2 ; 3)}$ as

$F_{\mathcal{O}_{2}}^{(1)}\left(1^{+}, 2^{+}, 3^{+} ; q\right):=2[12][23][31] \cdot f^{(2 ; 3)}\left(s_{12}, s_{23}, s_{13}\right)$,

with

$$
\begin{aligned}
f^{(2 ; 3)}\left(s_{12}, s_{23}, s_{13}\right)= & f_{6 \mathrm{D}}^{(2 ; 3)}\left(s_{12}, s_{23}, s_{13}\right) \\
& -2 f_{\phi}^{(2 ; 3)}\left(s_{12}, s_{23}, s_{13}\right),
\end{aligned}
$$

Notice that we decided not to normalize by the corresponding tree-level form-factor, which carries additional nontrivial dependence on the Mandelstam variables, but simply by a factor [12][23][31] which only captures the complete helicity dependence of the operator. Computing the discontinuity in the $s_{12}$-channel we have

$$
\begin{aligned}
\left.f_{6 \mathrm{D}}^{(3)}\left(\left\{s_{i j}\right\}\right)\right|_{s_{12}-\mathrm{cut}}= & \frac{1}{2[12][23][31]} \int \mathrm{d} \operatorname{LIPS} F_{\mathcal{O}_{2}}^{(0)}\left(l_{1}^{a \dot{a}}, l_{2}^{b \dot{b}}, 3_{1 \mathrm{i}}\right) \\
& \times \mathcal{A}^{(0)}\left(-l_{2 a \dot{a}},-l_{1 b \dot{b}}, 1_{1 \dot{1}}, 2_{2 \dot{2}}\right)
\end{aligned}
$$

which, upon making use of momentum conservation in the form of (4.5), is a 356-term expression. We make use of the redundant d.o.f. to simply the expression by choosing

$\mu_{l_{1}} \mapsto \lambda_{l_{2}}, \quad \tilde{\mu}_{l_{1}} \mapsto \tilde{\lambda}_{3}, \quad \mu_{l_{2}} \mapsto \lambda_{l_{1}}, \quad \tilde{\mu}_{l_{2}} \mapsto \tilde{\lambda}_{3}$,

which leads to

$$
\left.f_{6 \mathrm{D}}^{(3)}\left(\left\{s_{i j}\right\}\right)\right|_{s_{12}-\mathrm{cut}} \propto \int \mathrm{dLIPS}\left[3 l_{1}\right]\left\langle l_{1} l_{2}\right\rangle\left[l_{2} 3\right] .
$$

Note that, after using (4.16), the last expression apparently is no longer invariant with respect to little-group transformations of $l_{1}$ and $l_{2}$, since these transformations mix the $\lambda$ and $\mu$. In other words, looking at the numerator of (4.17), $l_{1}$ and $l_{2}$ appear as four-dimensional massless momenta, whereas they should really be massive. Hence in order to further manipulate the expression in a consistent manner we have to restore the masses, i.e., restore explicit littlegroup invariance. This is achieved by the replacement

$$
\lambda_{i}^{\alpha} \tilde{\lambda}_{i}^{\dot{\alpha}} \mapsto \underbrace{\left(\lambda_{i}^{\alpha} \tilde{\lambda}_{i}^{\dot{\alpha}}+\frac{\mu^{2}}{\left\langle\lambda_{i} \mu_{i}\right\rangle\left[\tilde{\mu}_{i} \tilde{\lambda}_{i}\right]} \mu_{i}^{\alpha} \tilde{\mu}_{i}^{\dot{\alpha}}\right)}_{p_{i}^{(4) \alpha \dot{\alpha}}}-\frac{\mu^{2}}{\left\langle\lambda_{i} \mu_{i}\right\rangle\left[\tilde{\mu}_{i} \tilde{\lambda}_{i}\right]} \mu_{i}^{\alpha} \tilde{\mu}_{i}^{\dot{\alpha}},
$$




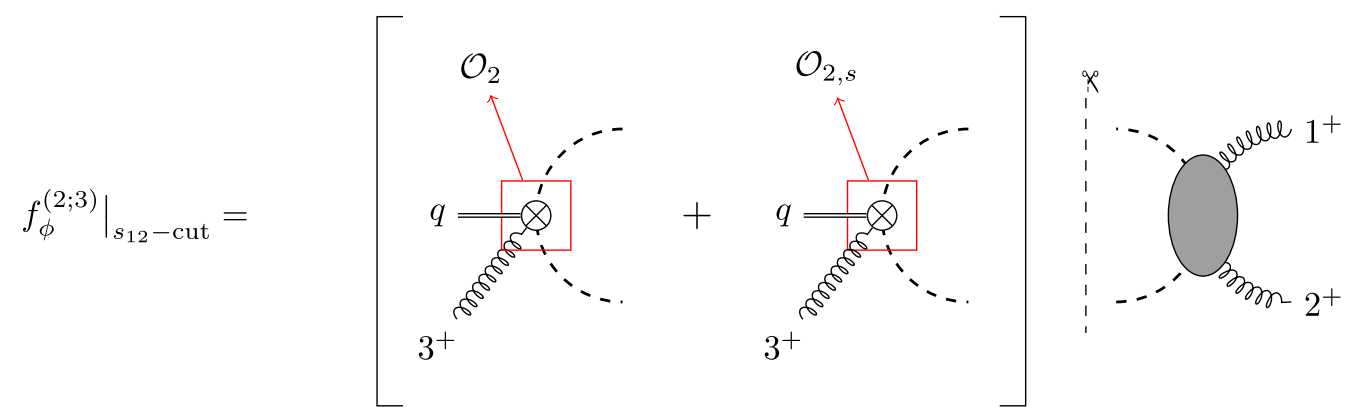

FIG. 4. A double cut of the scalar contribution to $\operatorname{Tr} F^{2}$ nonminimal. The red boxes highlight the two different operator insertions.

which in the particular case of (4.17) becomes

$$
\begin{aligned}
& \left|l_{1}\right\rangle\left[l_{1}\left|\mapsto l_{1}^{(4)}-\frac{\mu^{2}}{\left\langle l_{1} l_{2}\right\rangle\left[3 l_{1}\right]}\right| l_{2}\right\rangle[3 \mid, \\
& \left|l_{2}\right\rangle\left[l_{2}\left|\mapsto l_{2}^{(4)}-\frac{\mu^{2}}{\left\langle l_{2} l_{1}\right\rangle\left[3 l_{2}\right]}\right| l_{1}\right\rangle[3 \mid,
\end{aligned}
$$

where the replacements (4.16) have already been applied. After this substitution and some further manipulation, (4.17) becomes

$$
\begin{aligned}
& \left.f_{6 \mathrm{D}}^{(2 ; 3)}\left(s_{12}, s_{23}, s_{13}\right)\right|_{s_{12}-\mathrm{cut}} \\
& \quad=i \frac{[12]}{[23][31]} \int \mathrm{dLIPS}\left[3\left|\ell_{1}^{(4)} l_{2}^{(4)}\right| 3\right] \mathcal{I}_{6 \mathrm{D}}^{(2 ; 3)},
\end{aligned}
$$

where

$$
\mathcal{I}_{6 \mathrm{D}}^{(2 ; 3)}=\frac{q^{4} s_{12}-2 \mu^{2} q^{2} s_{12}-4 \mu^{2} s_{3 l_{1}} s_{3 l_{2}}}{s_{12}^{2} s_{2,-l_{2}} s_{3 l_{1}} s_{3 l_{2}}}
$$

Performing the appropriate scalar subtraction for the nonminimal configuration of the operator $\operatorname{Tr} F^{2}$ is more subtle than in the minimal case. The double cut one needs to compute is represented in Fig. 4. There are two different tree-level form factors to be inserted into the cut: the nonminimal form factors with two external scalars and one gluon of the operators $\operatorname{Tr} F^{2}$ and $(D \phi)^{2}$. The tree-level expression for these form factors are given in (3.14) and (3.15) respectively. Computing the complete result for the double-cut of the scalar contribution leads to

$$
\begin{aligned}
& \left.f_{\phi}^{(2 ; 3)}\left(s_{12}, s_{23}, s_{13}\right)\right|_{s_{12}-\mathrm{cut}} \\
& \quad=i \frac{[12]}{[23][31]} \int \mathrm{dLIPS}\left[3\left|\boldsymbol{l}_{1}^{(4)} \boldsymbol{l}_{2}^{(4)}\right| 3\right] \mathcal{I}_{\phi}^{(2 ; 3)},
\end{aligned}
$$

with

$$
\mathcal{I}_{\phi}^{(2 ; 3)}=-\mu^{2} \frac{q^{2} s_{12}+s_{3 l_{1}} s_{3 l_{2}}}{s_{12}^{2} s_{2,-l_{2}} s_{3 l_{1}} s_{3 l_{2}}}
$$

Upon subtracting twice (4.22) from (4.20), uplifting the cut and performing some algebraic manipulations on the numerator, one ends up with the final expression:

$$
f^{\left.(2,3)\left(s_{12}, s_{23}, s_{13}\right)\right|_{\mathrm{s}_{12}-\mathrm{disc}}=-\frac{i q^{4}}{2 s_{31}}}
$$

where all integrals can be found in Appendix E. 


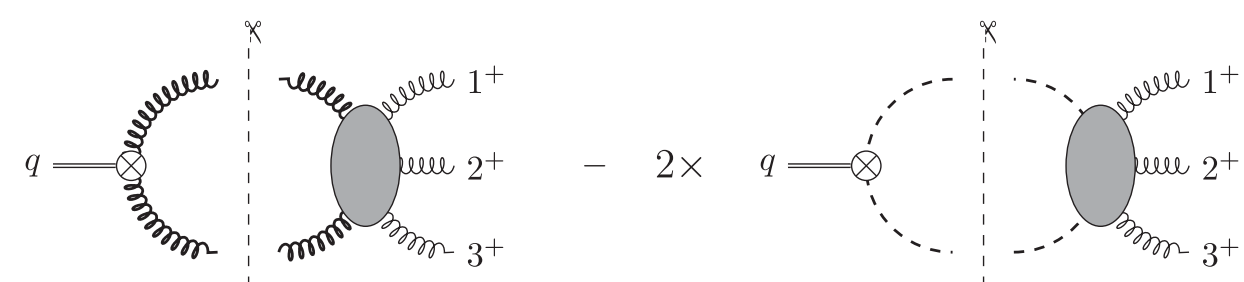

FIG. 5. Two-particle cut of the one-loop form factor $\operatorname{Tr} F^{2}$ in the $s_{123}$ channel in six dimensions.
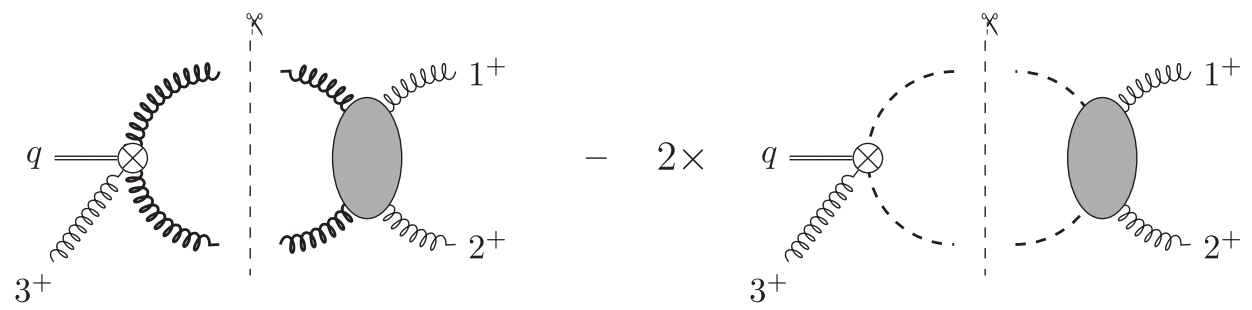

FIG. 6. Two-particle cut of the one-loop form factor $\operatorname{Tr} F^{3}$ in the $s_{12}$ channel in six dimensions.

Clearly the double cuts in the channels $s_{23}$ and $s_{13}$ can be derived from (4.24) by symmetry arguments, thus the only invariant channel left to compute would be $s_{123}$, see Fig. 5 . This double-cut involves the use of the five-point amplitudes in six dimensions with five gluons as well as with three gluons and two scalars, ${ }^{15}$ combined with the minimal form factor of $\mathcal{O}_{2}$ and $\mathcal{O}_{2, s}$ respectively. The only topology probed by this cut, which is not probed by any of the previous cuts, is the bubble with the form factor in one of the two vertices and all the momenta in the other. Performing the calculation the associated coefficient turns out to be zero. Thus (4.24) and its permutations give the complete result, which matches the one given in $[16,72]$.

\section{The minimal $\operatorname{Tr} F^{3}$ form factors}

We now consider the $\operatorname{Tr} F^{3}$ form factor in the all-plus helicity configuration. The procedure we follow is exactly the same as in the $\operatorname{Tr} F^{2}$ case. First we factor out the helicity dependence as an overall tree-level prefactor:

$$
\begin{aligned}
F_{\mathcal{O}_{3}}^{(1)}\left(1^{+}, 2^{+}, 3^{+} ; q\right) & :=F_{\mathcal{O}_{3}}^{(0)}\left(1^{+}, 2^{+}, 3^{+} ; q\right) \cdot f^{(3)}\left(s_{12}, s_{23}, s_{13}\right) \\
& =-2[12][23][31] \cdot f^{(3)}\left(s_{12}, s_{23}, s_{13}\right),
\end{aligned}
$$

then we compute $f^{(3)}$ as the difference $f_{6 \mathrm{D}}^{(3)}-2 f_{\phi}^{(3)}$. We start with the two-particle cut in the $s_{12}$ channel represented in Fig. 6, which reads

$$
\begin{aligned}
\left.f_{6 \mathrm{D}}^{(3)}\left(s_{12}\right)\right|_{s_{12}-\mathrm{cut}}= & -\frac{1}{2[12][23][31]} \\
& \times \int \mathrm{dLIPS} F_{\mathcal{O}_{3}}^{(0)}\left(-l_{1}^{a \dot{a}},-l_{2}^{b \dot{b}}, 3_{1 \dot{1}}\right) \\
& \times \mathcal{A}^{(0)}\left(l_{2 a \dot{a}}, l_{1 b \dot{b}}, 1_{1 \dot{1}}, 2_{2 \dot{2}}\right) .
\end{aligned}
$$

\footnotetext{
${ }^{15}$ Their analytic expression is given in Appendix C.
}

Upon expanding the six-dimensional invariants we get a 168-term expression. This can be considerably simplified using momentum conservation as in (4.5) and choosing the $\mu$ s to be

$\mu_{l_{1}} \mapsto \lambda_{l_{2}}, \quad \tilde{\mu}_{l_{1}} \mapsto \tilde{\lambda}_{3}, \quad \mu_{l_{2}} \mapsto \lambda_{l_{1}}, \quad \tilde{\mu}_{l_{2}} \mapsto \tilde{\lambda}_{3}$.

Doing so, we arrive at the compact expression

$$
\begin{aligned}
& \left.f_{6 \mathrm{D}}^{(3)}\left(s_{12}, s_{23}, s_{13}\right)\right|_{s_{12}-\mathrm{cut}} \\
& \quad=i \int \mathrm{dLIPS}\left(\frac{[12]\left[3\left|\ell_{1}^{(4)} l_{2}^{(4)}\right| 3\right]}{s_{2 l_{2}}[23][31]}+\mu^{2} \frac{\left[3\left|\ell_{1}^{(4)} \ell_{2}^{(4)}\right| 3\right]}{\left[3\left|\not p_{1} \not \not_{2}\right| 3\right]}\right),
\end{aligned}
$$

where we have already reconstructed the full $d$-dimensional momenta. Computing the scalar contribution in a similar fashion ${ }^{16}$ leads to

$\left.f_{\phi}^{(3)}\left(s_{12}, s_{23}, s_{13}\right)\right|_{s_{12}-\mathrm{cut}}=\frac{i}{2} \int \mathrm{dLIPS} \mu^{2} \frac{\left[3\left|\ell_{1}^{(4)} \eta_{2}^{(4)}\right| 3\right]}{\left[3\left|\not \not_{1} \not \not_{2}\right| 3\right]}$,

and finally

$\left.f^{(3)}\left(s_{12}, s_{23}, s_{13}\right)\right|_{s_{12}-\mathrm{cut}}=i \frac{[12]}{[23][31]} \int \mathrm{dLIPS} \frac{\left[3\left|\ell_{1}^{(4)} \ell_{2}^{(4)}\right| 3\right]}{s_{2 l_{2}}}$.

\footnotetext{
${ }^{16}$ For the case of the scalar contribution it turns out that the most convenient choice for the $\mu$ s is the same as in the gluon case. Notice that it is for this particular reason that we would have been allowed to perform the subtraction between the two contributions without writing them in terms of full $d$-dimensional quantities first. Indeed, if this were not the case, we would have had to reconstruct the form of the loop momenta in terms of general $\mu \mathrm{s}$ before doing the subtraction.
} 
After using (4.27), it is possible to write $f^{(3)}$ in terms of Mandelstam invariants:

$$
\left.f^{(3)}\left(s_{12}, s_{23}, s_{13}\right)\right|_{s_{12}-\text { cut }}=-i \int \mathrm{dLIPS}\left(\frac{s_{12}}{s_{2 l_{2}}}+2\right)
$$

modulo terms which integrate to zero. Uplifting this result leads to:

$$
\left.f^{(3)}\left(s_{12}, s_{23}, s_{13}\right)\right|_{s_{12}-\text { disc }}=-i s_{12} \cdot>_{p_{3}}^{q}
$$

Combining the discontinuities in the three channels $s_{12}, s_{23}$ and $s_{31}$ we arrive at the complete one-loop form factor

$$
f^{(3)}\left(\left\{s_{i j}\right\}\right)=\left.\sum_{k=1}^{n} f^{(3)}\left(\left\{s_{i j}\right\}\right)\right|_{s_{k k+1}-\text { disc }},
$$

where every term in the sum can be obtained from (4.32) by relabeling the external legs.

\section{The nonminimal $\operatorname{Tr} F^{3}$ form factor}

In the last sections we showed how the dimensional reconstruction can be applied to form factors. In this section we derive for the first time the complete form factor of the operator $\operatorname{Tr} F^{3}$ with four gluons in the all-plus helicity configuration.

The procedure we follow has been described in detail earlier, hence we now only sketch the relevant derivations and provide the main results. Up to cyclic permutations there are two independent unitarity cuts to be computed, say in the $s_{12^{-}}$ channel and $s_{123}$-channel. Starting from the $s_{123}$-cut, one needs to evaluate the following difference to obtain the complete result:
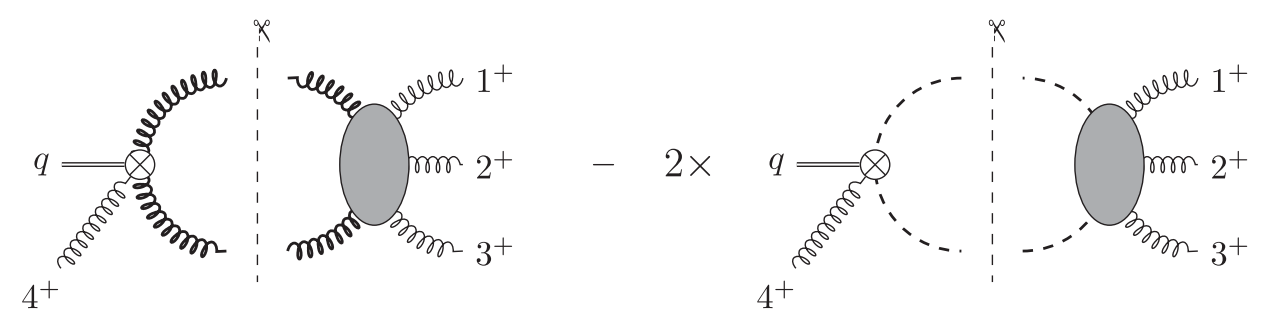

where the tree-level form factor in the scalar subtraction term (second term in the figure above) is the minimal form factor of the operator $\operatorname{Tr}\left(D_{\mu} \phi D_{\nu} \phi F^{\mu \nu}\right)$. The Passarino-Veltman reductions of the resulting tensor integrals have been performed using the Mathematica package FEYNCALC $[73,74]$. From the two-particle cuts in the $s_{123}$-channel we obtain the following functions:

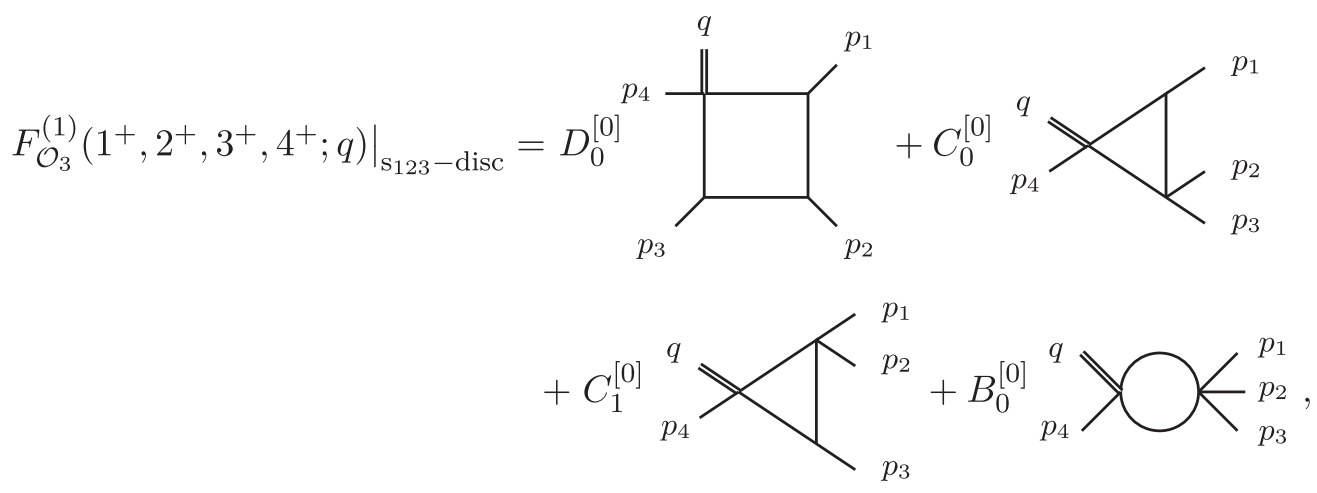

with the coefficients 


$$
\begin{aligned}
& D_{0}^{[0]}=-i \mathrm{~F}(1,2,3 ; 4), \\
& C_{0}^{[0]}=-i \frac{s_{12}+s_{31}}{s_{12} s_{23}} \mathrm{~F}(1,2,3 ; 4), \\
& C_{1}^{[0]}=-i \frac{s_{23}+s_{31}}{s_{12} s_{23}} \mathrm{~F}(1,2,3 ; 4),
\end{aligned}
$$

where

$$
\begin{aligned}
\mathrm{F}(1,2,3 ; 4):= & \frac{s_{123}}{s_{31}^{2}}\left(s_{12}[13][24]+s_{31}[12][34]\right)\left(s_{23}[13][24]\right. \\
& \left.+s_{31}[23][14]\right) .
\end{aligned}
$$

Finally the coefficient of the bubble can be written as

$$
B_{0}^{[0]}=2 i[12][23][34][41] \mathrm{b}_{0}^{[0]},
$$

where the helicity-blind function $\mathrm{b}_{0}^{[0]}$ is defined as

$$
\begin{aligned}
\mathrm{b}_{0}^{[0]}= & \frac{s_{123}^{2}}{s_{12} s_{23}}\left(\frac{1}{s_{12}+s_{31}}+\frac{1}{s_{23}+s_{31}}\right) \\
& +\frac{[13][24]}{[12][34]} \frac{s_{123}^{2}}{s_{23} s_{31}} \cdot \frac{1}{s_{12}+s_{31}} \\
& +\frac{[13][24]}{[14][23]} \frac{s_{123}^{2}}{s_{12} s_{31}} \cdot \frac{1}{s_{23}+s_{31}} .
\end{aligned}
$$

The result also contains a box integral with a $\mu^{2}$ i numerator, which after integration is of $\mathcal{O}(\epsilon)$. For completeness we quote its coefficient:

$D_{0}^{[2]}=-2 i s_{123}\left(\frac{[12]^{2}[34]^{2}}{s_{12}}+\frac{[23]^{2}[41]^{2}}{s_{23}}+\frac{[13]^{2}[24]^{2}}{s_{31}}\right)$.

Next we consider the two-particle cut in the $s_{12}$-channel and, as discussed in earlier sections, the discontinuity of the complete form factor is determined from the difference

$$
\begin{aligned}
& \left.F_{6 \mathrm{D}}^{(1)}\left(1^{+}, 2^{+}, 3^{+}, 4^{+} ; q\right)\right|_{s_{12}-\mathrm{cut}} \\
& \quad-\left.2 F_{\phi}^{(1)}\left(1^{+}, 2^{+}, 3^{+}, 4^{+} ; q\right)\right|_{s_{12}-\mathrm{cut}},
\end{aligned}
$$

where the second term is the scalar subtraction. As in the case of the nonminimal form factor of $\operatorname{Tr} F^{2}$, there are two contributions to the scalar quantity $F_{\phi}^{(1)}\left(1^{+}, 2^{+}, 3^{+}, 4^{+} ; q\right)$, which are represented in Fig. 7. The first contribution comes from the operator $\operatorname{Tr} F^{3}$ with two scalars and two gluons, whereas the second one comes from the scalar operator $\operatorname{Tr} D_{\mu} \phi D_{\nu} \phi F^{\mu \nu}$.

After tensor reductions, we find

$$
\left.F_{\mathcal{O}_{3}}^{(1)}\left(1^{+}, 2^{+}, 3^{+}, 4^{+} ; q\right)\right|_{\mathrm{S}_{12} \text { disc }}=D_{0}^{[0]}
$$




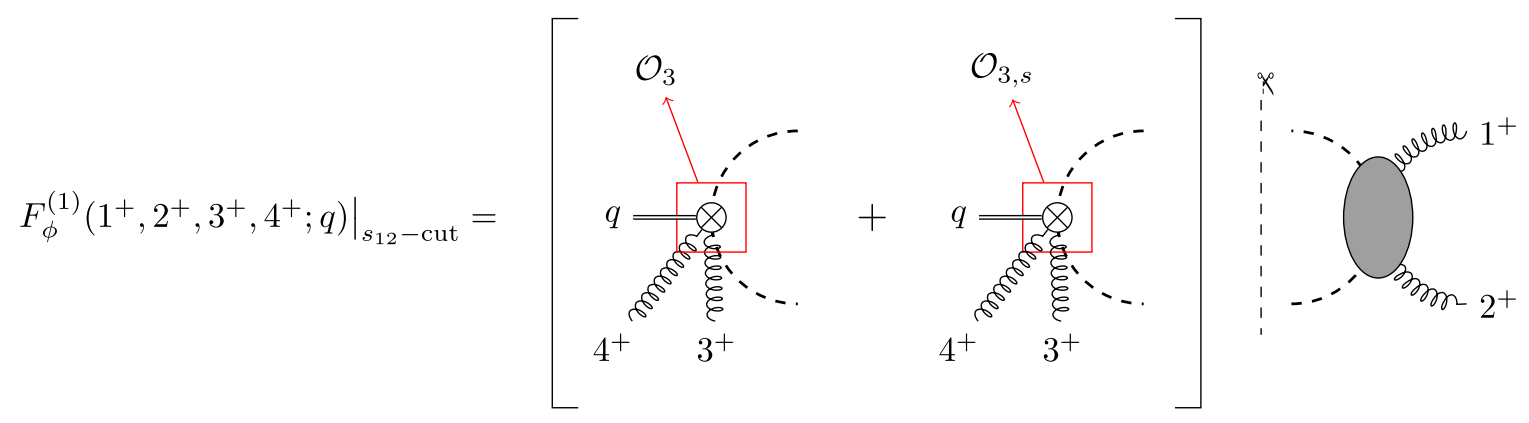

FIG. 7. A two-particle cut of the scalar contribution to the nonminimal $\operatorname{Tr} F^{3}$ form factor. The red boxes highlight the two different operator insertions.

where we checked that the coefficients $D_{0}^{[p]}, D_{1}^{[p]}, C_{0}^{[p]}$ and $C_{2}^{[p]}$ match the ones found in the previous calculation, up to relabeling. The other coefficients for the triangles are

$$
\begin{aligned}
& C_{3}^{[0]}=i[12][23][34][41] \mathrm{c}_{3}^{[0]}, \\
& C_{3}^{[2]}=\frac{4 i}{s_{12}}[12][34][13][24],
\end{aligned}
$$

where

$$
\begin{aligned}
c_{3}^{[0]}= & \frac{s_{12}+s_{31}}{s_{23}}+\frac{s_{12}}{s_{34}}\left(1+\frac{s_{13}}{s_{14}}+\frac{s_{24}}{s_{23}}\right) \\
& -\frac{[13][24]}{[14][23]}\left[\frac{s_{123}\left(s_{12}+s_{31}\right)-s_{13}^{2}}{s_{13}^{2}}-\frac{s_{12}}{s_{34}}\right] \\
& -\frac{[13][24]}{[12][33]} \frac{s_{12}}{s_{13}^{2} s_{23}}\left[s_{123}\left(s_{23}+s_{31}\right)-2 s_{31}^{2}\right] \\
& +(1,4) \leftrightarrow(2,3),
\end{aligned}
$$

while for the bubbles

$$
\begin{aligned}
B_{1}^{[0]}= & 2 i[13]^{2}[24]^{2}\left(\frac{1}{s_{31}}-\frac{1}{s_{23}}+\frac{s_{12}}{s_{23} s_{31}}\right) \\
& +2 i[12]^{2}[34]^{2}\left(\frac{2}{s_{23}}-\frac{2 s_{12}}{s_{23}\left(s_{13}+s_{23}\right)}\right) \\
& +2 i[12][34][13][24]\left(\frac{1}{s_{12}}+\frac{4}{s_{23}}-\frac{2}{s_{34}}-\frac{4 s_{24}}{s_{23} s_{34}}\right) \\
& +(1,4) \leftrightarrow(2,3),
\end{aligned}
$$

and

$$
B_{1}^{[2]}=\frac{4 i}{s_{12}^{2}}[12][34]([13][24]+[23][14]) .
$$

We have checked that our result satisfies the expected infrared consistency conditions. In particular, using the results for the coefficients $D_{0}, C_{0}$ and $C_{1}$, one immediately finds that the coefficient of $\frac{\left(-s_{123}\right)^{-\epsilon}}{\epsilon^{2}}$ vanishes, as required.
We have also confirmed that the coefficient of $\frac{\left(-s_{12}\right)^{-\epsilon}}{\epsilon^{2}}$ is proportional to the corresponding tree-level nonminimal form factor derived in [37],

$$
\begin{aligned}
& F_{\mathcal{O}_{3}}^{(0)}\left(1^{+}, 2^{+}, 3^{+}, 4^{+} ; q\right) \\
& \quad=-2 \frac{[12][23][34][41]}{s_{12}}\left(1+\frac{[13][24]}{[23][41]}-\frac{s_{24}}{s_{41}}\right)+\text { cyclic. }
\end{aligned}
$$

\section{E. The minimal $\operatorname{Tr} F^{4}$ form factors}

In this section we consider the form factors of $\operatorname{Tr} F^{4}$ in all possible helicity configurations. The case where all particles have the same helicity is interesting since it admits an immediate generalization to the minimal form factors of operators of the form $\operatorname{Tr} F^{n}$ defined in (3.35). In this family, $\operatorname{Tr} F^{4}$ is the first operator whose minimal form factor contains rational terms. We are going to consider the quantities in the planar limit of the theory, i.e., at one loop we will probe only the discontinuities in the Mandelstam invariants of adjacent momenta in the color-ordered form factor. At this point it is important to stress that nonplanar contributions behave differently: as one can see from (2.10) there is no nonplanar scalar contribution, because in the operator the scalars can only appear next to each other, and then the complete four-dimensional contribution coincides with the diagrams with purely six-dimensional internal gluons.

\section{All-plus felicity configuration}

We begin by defining

$F_{\mathcal{O}_{4}}^{(1)}\left(1^{+}, 2^{+}, 3^{+}, 4^{+} ; q\right):=2[12][23][34][41] \cdot f^{(4)}\left(\left\{s_{i j}\right\}\right)$.

At one loop, we can make the following observations:

(i) The cut-constructible part, coming from the form factor involving only gluons, has the same structure as $F_{\mathcal{O}_{3}}^{(1)}\left(1^{+}, 2^{+}, 3^{+} ; q\right)$, with both $\mathrm{UV}$ and IR divergences. 
(ii) Terms proportional to $\mu^{2}$ and $\mu^{4}$ now appear. As already mentioned, these could not arise for $n<4$ because of the limited kinematic, as we will show below. The new integrals are two triangles with $\mu^{2}$ and $\mu^{4}$ numerators ${ }^{17}$ and when expanded in powers of the dimensional regulator $\epsilon$ give a finite contribution in the $\epsilon \rightarrow 0$ limit. They are exactly the rational terms that cannot be seen by the completely four-dimensional cut construction, where clearly $\mu^{2}=0$.

Following the procedure outlined in the previous sections, we find

$$
\left.f^{4}\left(\left\{s_{i j}\right\}\right)\right|_{\mathrm{s}_{12}-\mathrm{disc}}=-i\left(1+\frac{[13][24]}{[14][23]}\right) \cdot p_{p_{3}}
$$

Notice that in the final result the integral $I_{3}^{4}\left[\mu^{4}\right]$ appears. In general, in a renormalizable gauge theory one would expect triangle integrals to appear with at most a third power of the loop momentum in the numerator, which allows for at most a $\mu^{2}$ triangle contribution. However we are considering an effective field theory with an operator of mass-dimension eight, hence the possibility of having also an $I_{3}^{4}\left[\mu^{4}\right]$ term. The last step of the calculation is the sum over all the possible channel discontinuities, as we did in (4.33) for $\operatorname{Tr} F^{3}$.
The above result can be immediately generalized to $\operatorname{Tr} F^{n}$ for arbitrary $n$ in the all-plus helicity configurations, where we define

$\left.\operatorname{Tr} F^{n}\left(1^{+}, \ldots, n^{+} ; q\right)\right|_{1-\text { loop }}:=(-)^{n} 2 \prod_{k=1}^{n}[k k+1] \cdot f^{(n)}\left(\left\{s_{i j}\right\}\right)$,

and

$$
\left.f^{(n)}\left(\left\{s_{i j}\right\}\right)\right|_{\mathrm{s}_{12}-\mathrm{disc}}=-i\left(1+\frac{[13][2 n]}{[1 n][23]}\right) \cdot{ }_{p_{3}}
$$

This simple generalization is due to the fact that, upon properly normalizing with the corresponding four-dimensional quantities, the six-dimensional minimal tree-level form factor of $\operatorname{Tr} F^{n}$ is identical to that of $\operatorname{Tr} F^{4}$ up to the replacement $4 \mapsto n$, as can be seen from (3.40). As a final remark, notice that we can a posteriori explain the absence of rational terms for $\operatorname{Tr} F^{3}$ : indeed we can recover (4.32) by simply replacing $n \mapsto 3$ in (4.50). Then, rational terms vanish since they are proportional to $[3 n]$.

\footnotetext{
${ }^{17}$ For analytic expressions of such integrals see for example Appendix E.
}

\section{MHV configuration: The alternate and split-helicity color ordered form factors}

We define the MHV color-ordered form factor with alternate-helicity gluons as follows:

$F_{\mathcal{O}_{4}}^{(1)}\left(1^{+}, 2^{-}, 3^{+}, 4^{-} ; q\right):=\langle 24\rangle^{2}[13]^{2} \cdot f_{a}^{(4)}\left(\left\{s_{i j}\right\}\right)$

Since this case presents some peculiarities in the calculations, we will give more details about it. In particular, the cut of the form factor with six-dimensional internal gluons in the $s_{12}$-channel is given by 


$$
\begin{aligned}
\left.f_{a, 6 D}^{(4)}\left(\left\{s_{i j}\right\}\right)\right|_{s_{12}-\mathrm{cut}} & =-\int \mathrm{d} \operatorname{LIPS} \frac{i}{s_{12} s_{2 l_{2}}} \frac{\mathcal{I}_{6 D}^{2}}{\langle 24\rangle^{2}[13]^{2}} \\
& =-\int \mathrm{dLIPS} \frac{i}{s_{12} s_{2 l_{2}}}\left(2 k \cdot l_{2}\right)^{2},
\end{aligned}
$$

where

$$
\begin{aligned}
\mathcal{I}_{6 D}= & 2 \mu^{2}\langle 24\rangle[13]+\left\langle 2\left|\ell_{1}^{(4)}\right| 3\right]\left\langle 4\left|\ell_{2}^{(4)}\right| 1\right] \\
& +\left\langle 2\left|\ell_{2}^{(4)}\right| 3\right]\left\langle 4\left|\ell_{1}^{(4)}\right| 1\right]
\end{aligned}
$$

and in the last step we removed terms proportional to $\left\langle 2\left|\ell_{2}^{(4)}\right| 1\right]$ that vanish upon integration. Also $k_{\mu}$ is a massive momentum defined by

$$
k_{\alpha \dot{\alpha}}=\frac{[12]}{[13]} \lambda_{2 \alpha} \tilde{\lambda}_{3 \dot{\alpha}}-\frac{\langle 12\rangle}{\langle 24\rangle} \lambda_{4 \alpha} \tilde{\lambda}_{1 \dot{\alpha}}
$$

and it is easy to prove that it satisfies the following relations:

$$
k^{2}=2 p_{1} \cdot k=2 p_{2} \cdot k=s_{12} .
$$

Surprisingly, the scalar contribution is identically zero after integration:

$$
\begin{aligned}
& \left.f_{a, \phi}^{(4)}\left(\left\{s_{i j}\right\}\right)\right|_{s_{12}-\mathrm{cut}} \\
& \quad=\int \mathrm{dLIPS} \frac{i}{s_{12} s_{2 l_{2}}} \frac{\left\langle 4\left|\ell_{1}^{(4)}\right| 3\right]\left\langle 4\left|\ell_{2}^{(4)}\right| 3\right]\left\langle 2\left|\ell_{2}^{(4)}\right| 1\right]^{2}}{\langle 24\rangle^{2}[13]^{2}}=0,
\end{aligned}
$$

because of the presence of the term $\left\langle 2\left|\ell_{2}^{(4)}\right| 1\right]^{2}$. Thus the discontinuity in the $s_{12}$-channel is completely given by the pure six-dimensional contribution (4.52), which after the integral reduction can be written as

$$
\left.f_{a}^{(4)}\left(\left\{s_{i j}\right\}\right)\right|_{s_{12}-\operatorname{disc}}=-i s_{12} \cdot{ }_{p_{3}}^{q} \text {. }
$$

It is worth stressing that all the other planar contributions can be obtained from the previous one easily by symmetry arguments.

As usual, for the split-helicity configuration we factorize the tree-level form factor:

$F_{\mathcal{O}_{4}}^{(1)}\left(1^{+}, 3^{+}, 2^{-}, 4^{-} ; q\right):=[13]^{2}\langle 24\rangle^{2} \cdot f_{s}^{(4)}\left(\left\{s_{i j}\right\}\right)$.

Unlike the previous case, in the planar limit we have two different cuts which cannot be related by symmetry: in particular, we can perform the cut in channels with same or opposite helicity gluons. The discontinuity in the $s_{12^{-}}$ channel, after the scalar subtraction, is given by

$$
\left.f_{s}^{(4)}\left(\left\{s_{i j}\right\}\right)\right|_{s_{13}-\text { disc }}=-i s_{13} \cdot{ }_{p_{2}}^{q}
$$

The cut in the $s_{23}$-channel is reminiscent of the alternatehelicity case, with vanishing scalar contribution up to integration:

$\left.f_{s}^{(4)}\left(\left\{s_{i j}\right\}\right)\right|_{s_{23}-\mathrm{cut}} \simeq-\int \mathrm{dLIPS} \frac{i}{s_{13} s_{3 l_{2}}}\left(2 k \cdot l_{2}\right)^{2}$,

where the momentum $k_{\mu}$ is defined by

$$
k_{\alpha \dot{\alpha}}=\frac{[23]}{[13]} \lambda_{2 \alpha} \tilde{\lambda}_{1 \dot{\alpha}}+\frac{\langle 23\rangle}{\langle 24\rangle} \lambda_{4 \alpha} \tilde{\lambda}_{3 \dot{\alpha}}
$$

and it satisfies the following relations:

$$
k^{2}=2 p_{2} \cdot k=2 p_{3} \cdot k=s_{23} .
$$

The cut in the $s_{23}$ channel is

$$
\left.f_{s}^{(4)}\left(\left\{s_{i j}\right\}\right)\right|_{s_{23}-\text { disc }}=-i s_{23} \cdot{ }_{p_{4}}^{q}
$$

Let us emphasize some relevant features of the result:

(i) The final result is free of rational terms. Thus we would have found the same, complete, quantity even with four-dimensional unitarity-cuts.

(ii) The only operator that contributes in four dimensions is $\operatorname{Tr}\left(F_{\mathrm{SD}}^{2} F_{\mathrm{ASD}}^{2}\right)$, which is a descendant of $\operatorname{Tr} \phi^{4}$ in $\mathcal{N}=4 \mathrm{SYM}^{18}$.

(iii) We note the absence of bubbles in the final result for this (unrenormalized) form factor. This may be related to the independence of the bare quantity on the matter content of the theory. One could then regard the computation as if it was performed in $\mathcal{N}=4$ SYM, where the operator under consideration belongs to a protected multiplet.

(iv) An unrelated observation is that the color-ordered form factors with alternate and split-helicity configurations are the same:

\footnotetext{
${ }^{18}$ See for example Table 7 in [75].
} 
$F_{\mathcal{O}_{4}}^{(1)}\left(1^{+}, 2^{-}, 3^{+}, 4^{-} ; q\right)=F_{\mathcal{O}_{4}}^{(1)}\left(1^{+}, 3^{+}, 2^{-}, 4^{-} ; q\right)$

This is an accident due to the simple topology of the integral basis combined with the fact that bubbles do not appear. At first, the equality (4.64) could appear as a consequence of the photon decoupling identities which hold in Yang-Mills theory. However these identities are no longer valid when one considers interactions with higher powers of the field strength.

\section{ACKNOWLEDGMENTS}

We would like to thank Lorenzo Coccia, Harald Ita, Rodolfo Panerai, Sebastian Pögel, and in particular Lorenzo Bianchi for interesting discussions. This work was supported by the Science and Technology Facilities Council (STFC) Consolidated Grant ST/P000754/1 "String theory, gauge theory \& duality", and by the European Union's Horizon 2020 research and innovation programme under the Marie Skłodowska-Curie Grant Agreement No. 764850 "SAGEX".

\section{APPENDIX A: FOUR-DIMENSIONAL SPINOR HELICITY FORMALISM}

In this section we briefly review the four-dimensional spinor helicity formalism (SHF) [76-79], having as a main goal to present our notation and conventions.

$\mathrm{SL}(2, \mathbb{C})$ is the universal covering of the Lorentz group $\mathrm{SO}^{+}(1,3)$. This means that the projective representations of $\mathrm{SO}(1,3)$ on the Hilbert space are in one-to-one correspondence to the unitary representations of $\operatorname{SL}(2, \mathbb{C})$. Furthermore, these infinite-dimensional unitary representations on the states naturally induces finite-dimensional representations on the operators (e.g., the fields) of the theory. Moreover, group theory ensures that all finite dimensional irreducible representations of $\operatorname{SL}(2, \mathbb{C})$ can be found by taking the totally symmetric tensor product of a finite number of its fundamental and antifundamental representations. The finite dimensional irreducible representations, labeled by two semi-integer numbers $(m, n),{ }^{19}$ are obtained by the symmetric tensor product of the representations $\left(\frac{1}{2}, 0\right)$ and $\left(0, \frac{1}{2}\right)$, respectively $2 m$ and $2 n$ times.

The fundamental objects transforming in the $\left(\frac{1}{2}, 0\right)$ representation will be labeled by $\lambda$ and the associated indices will be undotted Greek letters as $\alpha, \beta, \ldots$. The fundamental objects transforming in the $\left(0, \frac{1}{2}\right)$ will be labeled by $\tilde{\lambda}$ with associated dotted Greek indices $\dot{\alpha}, \dot{\beta}, \ldots$.

What group theory states is that we can construct any quantity transforming in some representation of the Lorentz group in a uniform way in terms of objects transforming in these two representations. In general any scattering

\footnotetext{
${ }^{19}$ In our conventions, $(m, n)$ has dimension $(2 m+1)(2 n+1)$.
}

amplitude can be written as a function of the set $\left\{\lambda_{i \alpha}, \tilde{\lambda}_{i \dot{\alpha}}\right\}$, with the index $i$ running over all in- and outgoing particles of the considered process. In order to level out the description we will take all particles as outgoing from now on. We will refer to $\lambda$ and $\tilde{\lambda}$ collectively as helicity spinors. In real momentum space, these two representations are related by complex conjugation.

Dotted and undotted indices are raised and lowered through the Levi-Civita tensor $\epsilon$ and we adopt the following conventions:

$$
\lambda^{\alpha}=\epsilon^{\alpha \beta} \lambda_{\beta}=\epsilon^{\alpha \beta} \epsilon_{\beta \gamma} \lambda^{\gamma} \rightarrow \epsilon_{\alpha \gamma} \gamma^{\gamma \beta}=\delta_{\alpha}^{\beta} .
$$

The same is true for dotted indices as well. Lorentz singlets can be build out of contractions of helicity spinors. Namely, our conventions for the angle and the square Lorentz invariant brackets are as follows:

$$
\begin{gathered}
\langle i j\rangle:=\left\langle\lambda_{i} \lambda_{j}\right\rangle:=\lambda_{i}^{\alpha} \lambda_{j \alpha}=-\left\langle\lambda_{j} \lambda_{i}\right\rangle, \\
{[i j]:=\left[\lambda_{i} \lambda_{j}\right]:=\tilde{\lambda}_{i \dot{\alpha}} \tilde{\lambda}_{j}^{\dot{\alpha}}=-\left[\lambda_{j} \lambda_{i}\right] .}
\end{gathered}
$$

It is simple to convince oneself that the so called Schouten identity holds:

$$
\langle i j\rangle \lambda_{k \alpha}+\langle j k\rangle \lambda_{i \alpha}+\langle k i\rangle \lambda_{j \alpha}=0 .
$$

An similar identity can be written for the $\tilde{\lambda}$ 's as well.

\section{Massless momenta}

Considering Lorentz vectors, one has that the vector representation of $\mathrm{SO}(1,3)$ corresponds to the $\left(\frac{1}{2}, \frac{1}{2}\right)$ representation of $\operatorname{SL}(2, \mathbb{C})$. This correspondence is explicitly given by

$$
p^{\mu} \rightarrow p_{\alpha \dot{\alpha}}:=p_{\mu} \sigma_{\alpha \dot{\alpha}}^{\mu}
$$

The sigma matrices satisfy the Clifford algebra

$$
\left\{\sigma_{\mu}, \bar{\sigma}_{\nu}\right\}=2 \eta_{\mu \nu}
$$

where $\bar{\sigma}^{\mu \dot{\alpha} \alpha}:=\epsilon^{\alpha \beta} \epsilon^{\dot{\alpha} \dot{\beta}} \sigma_{\beta \dot{\beta}}^{\mu}$. Then, if we further define $p^{\dot{\alpha} \alpha}:=p^{\mu} \bar{\sigma}_{\mu}^{\dot{\alpha} \alpha}$, we have that

$$
p_{i}^{\dot{\alpha} \alpha} p_{j \alpha \dot{\alpha}}=2 p_{i} \cdot p_{j} .
$$

For a massive particle the mass-shell condition can then be written as

$$
p^{2}=m^{2} \rightarrow p^{\dot{\alpha} \alpha} p_{\alpha \dot{\alpha}}=\operatorname{det}\left(p_{\alpha \dot{\alpha}}\right)=m^{2} .
$$

The power of the SHF becomes manifest when we consider massless momenta. Indeed, the massless condition can be trivialized by picking 


$$
p_{i \alpha \dot{\alpha}}=\lambda_{i \alpha} \tilde{\lambda}_{i \dot{\alpha}}
$$

There is an ambiguity in the definition of $p_{i}$ in terms of $\left\{\lambda_{i}, \tilde{\lambda}_{i}\right\}$, which is represented by a phase

$$
\lambda_{i \alpha} \rightarrow e^{-i \varphi_{i}} \lambda_{i \alpha}, \quad \tilde{\lambda}_{i \dot{\alpha}} \rightarrow e^{i \varphi_{i}} \tilde{\lambda}_{i \dot{\alpha}} .
$$

This rescaling leaves momentum invariant and is thus a little group transformation. The little group in four dimensions is the double covering of $\mathrm{SO}(2) \simeq \mathrm{U}(1)$ and we choose to assign helicity $-\frac{1}{2}$ to $\lambda$ and $+\frac{1}{2}$ to $\tilde{\lambda}$. Thus it is now manifest how the new variables can carry information about both the momentum and the helicity of an associated particle.

\section{Massive momenta}

At this point we turn our attention to the spinor helicity description of massive momenta, of which we make extensive use. One can always write a massive momentum $L$ as [80]

$$
L^{\mu}=l^{\mu}+\frac{L^{2}}{2 l \cdot \eta} \eta^{\mu}
$$

where both $l$ and $\eta$ are massless momenta and $L^{2}=m^{2}$ is the mass associated to this momentum. The previous expression fixes $l^{\mu}$ in terms of the massive momentum $L^{\mu}$ completely once we have chosen the arbitrary $\eta^{\mu}$. We can write (A10) in terms of helicity spinors as

$$
p_{i \alpha \dot{\alpha}}=\lambda_{i \alpha} \tilde{\lambda}_{i \dot{\alpha}}+\frac{m^{2}}{\left\langle\lambda_{i} \mu_{i}\right\rangle\left[\tilde{\mu}_{i} \tilde{\lambda}_{i}\right]} \mu_{i \alpha} \tilde{\mu}_{i \dot{\alpha}}
$$

Focusing on the number of d.o.f. we expect to have 3 d.o.f. from the spinor variables, plus an additional d.o.f. from the mass squared $m^{2}$. The quantity $\lambda_{\alpha} \tilde{\lambda}_{\dot{\alpha}}$ already carries by itself 3 d.o.f., but $\mu_{\alpha}$ and $\tilde{\mu}_{\dot{\alpha}}$ apparently carry two additional complex degrees, which coincide with their direction, while the momentum is invariant under the rescaling

$$
\mu_{\alpha} \rightarrow a \mu_{\alpha}, \quad \tilde{\mu}_{\dot{\alpha}} \rightarrow b \tilde{\mu}_{\dot{\alpha}},
$$

where $a, b \in \mathbb{C}$. The redundancy is taken into account by the four-dimensional massive little group $\widetilde{\mathrm{SO}}(3) \simeq \mathrm{SU}(2)$, which has two additional generators, with respect to the massless one. Indeed we can write the massive momentum in terms of the irreducible $\mathrm{SU}(2)$ helicity spinors [81]

$$
\lambda_{\alpha}^{I}=\left(\lambda_{\alpha} \frac{m}{\langle\lambda \mu\rangle} \mu_{\alpha}\right)
$$

where $I$ is an index in the fundamental of $\mathrm{SU}(2)$ and

$$
p_{\alpha \dot{\alpha}}=\lambda_{\alpha}^{I} \tilde{\lambda}_{\dot{\alpha} I}=\epsilon_{I J} \lambda_{\alpha}^{I} \tilde{\lambda}_{\dot{\alpha}}^{J},
$$

where $\tilde{\lambda}_{\dot{\alpha} I}= \pm\left(\lambda_{\alpha}^{I}\right)^{\dagger}$, according to the sign of the $p_{0}$ component of momentum. In this form it is obvious that any $\mathrm{SU}(2)$ transformation

$$
\lambda_{\alpha}^{I} \rightarrow \lambda_{\alpha}^{J} U_{J}^{I}
$$

leaves the momentum invariant.

\section{APPENDIX B: SIX-DIMENSIONAL SPINOR HELICITY FORMALISM}

In this section we give a concise overview of the sixdimensional spinor helicity formalism. In particular we are interested in how it can be broken down in terms of a fourdimensional subspace and the associated four-dimensional spinors. For a more detailed discussion see $[14,17,62,82]$.

\section{Helicity spinors in six dimensions}

In six-dimensional Minkowski spacetime, the Lorentz group is $\mathrm{SO}(1,5)$, whose universal covering group is $\mathrm{SL}(2, \mathbb{U})$, and we will denote it as $\mathrm{SU}^{*}(4)$. Indeed, its representations are in one-to-one correspondence to those of $\mathrm{SU}(4)$, which is the universal covering of $\mathrm{SO}(6)$. The sixdimensional little group is $\tilde{\mathrm{SO}}(4) \simeq \mathrm{SU}(2) \times \mathrm{SU}(2)$.

Let us denote with $\square^{A}$ and $\square_{A}$ the objects transforming respectively in the fundamental and anti-fundamental representations of the Lorentz group $\mathrm{SU}^{*}(4)$ and $(a, \dot{a})$ the indices of the bifundamental representations of the two components of the little group. The Clifford algebra is defined by

$$
\left\{\gamma^{\mu}, \tilde{\gamma}^{\nu}\right\}_{A} B:=\gamma_{A C}^{\mu} \tilde{\gamma}^{\nu C B}+\gamma_{A C}^{\nu} \tilde{\gamma}^{\mu C B}=2 \eta^{\mu \nu} \delta_{A}^{B},
$$

where $\mu=0, \ldots, 6, \gamma_{A B}^{\mu} \equiv \gamma_{[A B]}^{\mu}$ and $\tilde{\gamma}^{\mu A B} \equiv \tilde{\gamma}^{\mu[A B]}$. These gamma matrices transform in the pseudoreal representation $\mathbf{6}=\mathbf{4} \wedge \mathbf{4}$ of $\mathrm{SU}^{*}(4)$ and are related by

$$
\tilde{\gamma}^{\mu A B}=\left(\gamma_{A B}^{\mu}\right)^{*}=\frac{1}{2} \epsilon^{A B C D} \gamma_{A B}^{\mu} .
$$

Six-dimensional momenta can be written as

$$
p_{A B}:=p_{\mu} \gamma_{A B}^{\mu},
$$

and also transform in the 6 representation. The massless condition on the momenta reads

$$
p^{2} \sim \epsilon^{A B C D} p_{A B} p_{C D}=0
$$

which can be solved by expressing the momentum as the bispinor

$$
p_{A B}=\epsilon^{\dot{a} \dot{b}} \tilde{\lambda}_{\dot{a} A} \tilde{\lambda}_{\dot{b} B}=\tilde{\lambda}_{\dot{a} A} \tilde{\lambda}_{B}^{\dot{a}},
$$

where $\tilde{\lambda}_{\dot{a} A}$ is a pseudoreal spinor. Analogously, we can write 


$$
p^{A B}=\lambda^{a A} \lambda_{a}^{B}=-\epsilon^{a b} \lambda_{a}^{A} \lambda_{b}^{B},
$$

which satisfies

$$
p^{A B}=\left(p_{A B}\right)^{*}=-\frac{1}{2} \epsilon^{A B C D} p_{C D} .
$$

Notice that, given the above definitions, the spinors $\lambda_{a A}$ and $\tilde{\lambda}_{\dot{a} A}$ automatically satisfy the Dirac equation:

$p_{A B} \lambda_{a}^{B}=-\frac{1}{2} \epsilon_{A B C D} \lambda_{a}^{B} \lambda^{b C} \lambda_{b}^{D}=-\epsilon_{A B C D} \lambda_{a}^{B} \lambda_{1}^{C} \lambda_{2}^{D}=0$,

and similarly for $\tilde{\lambda}_{\dot{a} A}$. The Dirac equation can be also written equivalently as a relation between $\lambda$ and $\tilde{\lambda}$ :

$$
0=\lambda^{a A} \lambda_{a}^{B} \tilde{\lambda}_{B \dot{a}}=-\lambda_{1}^{A} \lambda_{2}^{B} \tilde{\lambda}_{B \dot{a}}+\lambda_{2}^{A} \lambda_{1}^{B} \tilde{\lambda}_{B \dot{a}},
$$

which implies

$$
\lambda_{a}^{A} \tilde{\lambda}_{A \dot{a}}=0 .
$$

Finally we need to define polarization vectors in terms of the spinors. Just as in four dimensions one has to introduce a reference spinor to do so, which we call $q$. Then a good definition is given by

$$
\begin{gathered}
\varepsilon_{a \dot{a}}^{A B}(p, q)=\frac{\sqrt{2}}{s_{p q}}\left|p_{a}\right\rangle^{[A}\left[p_{\dot{a}} \mid \phi^{B]},\right. \\
\left.\varepsilon_{a \dot{a} A B}(p, q)=-\frac{\sqrt{2}}{s_{p q}} \mid p_{\dot{a}}\right]_{[A}\left\langle p_{a}\right| \phi_{B]} .
\end{gathered}
$$

\section{2. $\mathrm{SU}^{*}(4)$ spinor identities}

In this subsection we present some useful identities for six-dimensional spinors. We focus on the $\mathrm{SU}^{*}(4)$ structure of the spinors and keep the little group indices implicit for the sake of clarity. Of course little-group indices can be restored at any time because they are unambiguously related to each spinor.

Consider a certain number of spinors $\lambda_{i}^{A}$ (and $\tilde{\lambda}_{i A}$ ), with labels $i=1, \ldots, n$. The Lorentz invariant objects which can be built out of these spinors are of three types:

(i) Bispinor invariant objects:

$$
\lambda_{i}^{A} \tilde{\lambda}_{j A}:=\langle i j]
$$

(ii) Two distinct four-spinors invariant objects:

$$
\begin{aligned}
\epsilon_{A B C D} \lambda_{i}^{A} \lambda_{j}^{B} \lambda_{k}^{C} \lambda_{l}^{D} & :=\langle i j k l\rangle, \\
\epsilon^{A B C D} \tilde{\lambda}_{i A} \tilde{\lambda}_{j B} \tilde{\lambda}_{k C} \tilde{\lambda}_{l D} & :=[i j k l] .
\end{aligned}
$$

The spinors transform in the fundamental representation of $\mathrm{SU}^{*}(4)$, thus $A=1, \ldots, 4$. Two identities (and their two complex conjugate) follow immediately from this:

$$
\lambda_{1}^{[A} \lambda_{2}^{B} \lambda_{3}^{C} \lambda_{4}^{D} \lambda_{5}^{E]}=0
$$

and

$$
\lambda_{1}^{[A} \lambda_{2}^{B} \lambda_{3}^{C} \lambda_{4}^{D]}=\frac{1}{4 !} \epsilon^{A B C D}\langle 1234\rangle,
$$

and analogous relations hold for $\tilde{\lambda}_{i A}$. Equations (B14) and (B15) can be combined to give the six-dimensional Schouten identity:

$$
\sum_{\text {cyclic }}\langle 1234\rangle \lambda_{5}^{A}=0
$$

\section{From six-dimensional to four-dimensional quantities}

For our purposes, we find it convenient to write sixdimensional spinors in terms of four-dimensional ones, allowing amplitudes to be expressed in terms of the more familiar four-dimensional spinors. We can view six-dimensional null vectors as four-dimensional massive ones, by defining the two complex mass parameters

$$
m:=p_{4}+i p_{5}, \quad \tilde{m}:=p_{4}-i p_{5},
$$

where $p_{4}$ and $p_{5}$ are the fifth and the sixth components of the $6 \mathrm{D}$ momentum $p_{\mu}$. The six-dimensional massless condition becomes then

$$
p^{2}=\left(p^{(4)}\right)^{2}-m \tilde{m}=0 .
$$

where $\left(p^{(4)}\right)^{2}=p_{0}^{2}-p_{1}^{2}-p_{2}^{2}-p_{3}^{2}$ is the four-dimensional massive momentum associated to $p_{\mu}$. We found it more efficient for our calculation to describe these momenta as a combination of two massless momenta, as in (A11). We can decompose 6D helicity spinors in terms of $4 \mathrm{D}$ spinors as

$\lambda_{a}^{A}=\left(\begin{array}{cc}-\frac{m}{\langle\lambda \mu} \mu_{\alpha} & \lambda_{\alpha} \\ \tilde{\lambda}^{\dot{\alpha}} & \frac{\tilde{m}}{[\mu \lambda]} \tilde{\mu}^{\dot{\alpha}}\end{array}\right), \quad \tilde{\lambda}_{A \dot{a}}=\left(\begin{array}{cc}\frac{\tilde{m}}{\langle\lambda \mu\rangle} \mu^{\alpha} & \lambda^{\alpha} \\ -\tilde{\lambda}_{\dot{\alpha}} & \frac{m}{[\mu \lambda]} \tilde{\mu}_{\dot{\alpha}}\end{array}\right)$,

where the little group indices label the columns and the $\mathrm{SU}^{*}(4)$ indices label the rows. The $\mathrm{SU}^{*}(4)$ index structure can be broken down into two $\operatorname{SL}(2, \mathbb{C})$ complex conjugated indices:

$$
\square^{A}=\left(\begin{array}{c}
\square_{\alpha} \\
\square^{\dot{\alpha}}
\end{array}\right), \quad \square_{A}=\left(\begin{array}{c}
\square^{\alpha} \\
\square_{\dot{\alpha}}
\end{array}\right) .
$$

This embedding is specific of our choice of gamma matrices: indeed, we choose them such that the $\gamma$-matrices restricted to 
$\mu=0, \ldots, 3$ reduce to the familiar chiral representation in four dimensions ${ }^{20}$.

$p^{A B}$ and $p_{A B}$ are invariant under the little group $\mathrm{SU}(2) \times$ $\mathrm{SU}(2)$ transformations

$$
\begin{aligned}
\lambda_{a}^{\prime A} & =U_{a}^{b} \lambda_{b}^{A}, \quad \tilde{\lambda}_{A \dot{a}}^{\prime}=U_{\dot{a}}^{\dot{b}} \tilde{\lambda}_{A \dot{b}}, \\
\left(U_{a}^{b}, U_{\dot{a}}^{\dot{b}}\right) & \in \mathrm{SU}(2) \times \mathrm{SU}(2) .
\end{aligned}
$$

The 6D momentum in 4D components reads:

$$
p^{A B}=\left(\begin{array}{cc}
-m \epsilon_{\alpha \beta} & \lambda_{\alpha} \tilde{\lambda}^{\dot{\beta}}+\rho \mu_{\alpha} \tilde{\mu}^{\dot{\beta}} \\
-\tilde{\lambda}^{\dot{\alpha}} \lambda_{\beta}-\rho \tilde{\mu}^{\dot{\alpha}} \mu_{\beta} & \tilde{m} \epsilon^{\dot{\alpha} \dot{\beta}}
\end{array}\right),
$$

where $\rho=\frac{m \tilde{m}}{\langle\lambda \mu\rangle\langle\mu \lambda\rangle}$. We notice that $m$ and $\tilde{m}$ completely fix the diagonal components, thus they are little group invariant objects (this was obvious from their definitions). In our choice of gamma matrices, the off-diagonal components precisely coincide with the $4 \mathrm{D}$ massive momentum:

$$
p_{\alpha \dot{\alpha}}^{(4)}=\lambda_{\alpha} \tilde{\lambda}_{\dot{\alpha}}+\rho \mu_{\alpha} \tilde{\mu}_{\dot{\alpha}}, \quad\left(p^{(4)}\right)^{2}=m \tilde{m} .
$$

It is easy to see that the two copies of $\mathrm{SU}(2)$ of the $6 \mathrm{D}$ little group act in an identical way on the 4D momenta and we recover the usual massive little group: indeed, they depend only on the combination $m \tilde{m}$ and we can obtain dotted transformations from the undotted by simply replacing

$$
m \rightarrow-\tilde{m}, \quad \tilde{m} \rightarrow-m
$$

The Lorentz invariant quantities $\left\langle i_{a} j_{\dot{a}}\right],\left\langle i_{a} j_{b} k_{c} l_{d}\right\rangle$, $\left[i_{\dot{a}} j_{\dot{b}} k_{\dot{c}} l_{\dot{d}}\right]$ can be written in terms of four-dimensional angle and square brackets, once the helicity indices are fixed ( $a, b, c, d=1,2$ and $\dot{a}, \dot{b}, \dot{c}, \dot{d}=\mathrm{i}, \dot{2})$, by using the decomposition given in (B20) and decomposing $\epsilon_{A B C D} \sim$ $\sum \epsilon^{\alpha \beta} \epsilon_{\dot{\alpha} \dot{\beta}}$ and $\delta_{B}^{A}=\operatorname{diag}\left(\delta_{\alpha}^{\beta}, \delta_{\dot{\beta}}^{\dot{\alpha}}\right)$.

\section{APPENDIX C: SIX-DIMENSIONAL SCATTERING AMPLITUDES}

Six-dimensional tree-level amplitudes are the basic ingredients of our unitarity-based recipe. In this section we give the analytic expressions needed for our calculations and comment on how to recover four-dimensional expressions in a specific limit.

As we already mentioned, in six dimensions the notion of helicity is encoded in a tensorial structure, which must be reflected by the amplitudes. The advantage of this tensorial nature of helicity is that a single (tensorial) expression of the amplitude contains all the possible four-dimensional helicity configurations, when dimensional reduced. The drawback however is that one loses some of the simplicity which was peculiar to specific helicity configurations. In particular there is no concept of MHV amplitudes.

In the previous section we have chosen the embedding of the four dimensions into the six-dimensional space. Thus the four-dimensional helicity structure is embedded in the six-dimensional amplitudes. In general this represents a good consistency check for six-dimensional results. In fact for an appropriate limit these results must return their fourdimensional counterparts. More specifically, accordingly to our embedding, it turns out that states characterized by little-group indices $(1,1)$ and $(2,2)$ correspond to the positive and the negative helicity states in the four-dimensional limit $(m, \tilde{m} \rightarrow 0)$, because of representation we chose for the gamma matrices. On the other hand, in four dimensions the additional $(1,2)$ and $(2,1)$ components coincide with two 4D scalars.

The four-gluon amplitude, computed in [17], is

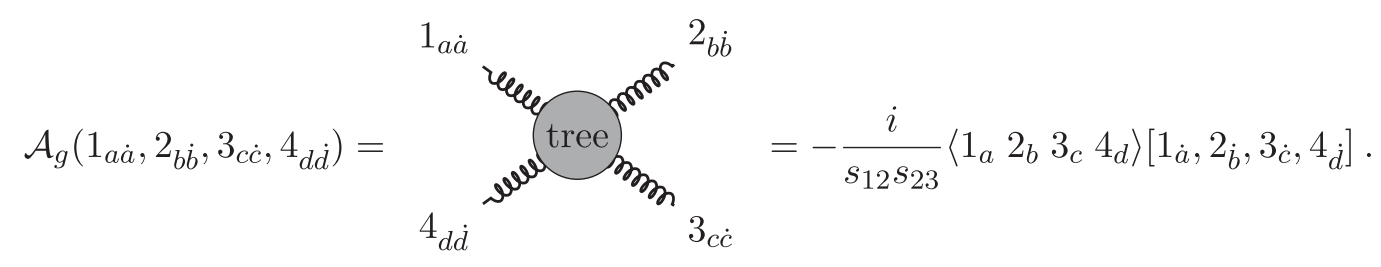

While $\mathcal{A}_{g}\left(1_{12}, 2_{21}, 3_{11}, 4_{22}\right)$ reproduces the four-point amplitude with two scalars and two opposite-helicity gluons $\mathcal{A}\left(1_{\phi}, 2_{\bar{\phi}}, 3^{+}, 4^{-}\right)$:

$$
\mathcal{A}_{g}\left(1_{12}, 2_{21}, 3_{11}, 4_{22}\right)=i \frac{\langle 14\rangle^{2}\langle 24\rangle^{2}}{\langle 12\rangle\langle 23\rangle\langle 34\rangle\langle 41\rangle} .
$$

Another amplitude of which we make frequent use is the six-dimensional four-point amplitude with two gluons and two scalars [16]

\footnotetext{
${ }^{20}$ For the explicit basis of gamma matrices see Appendix A of [17].
} 


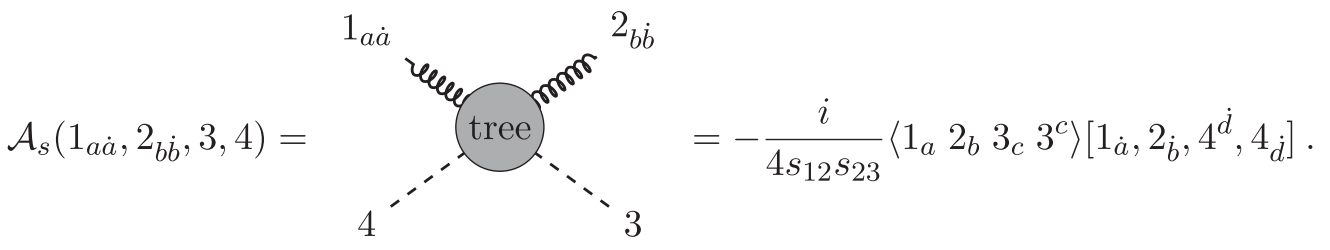

The massless scalars in six dimensions behave as massive scalars when reduced to four dimensions. Taking the limits $m_{1}, m_{2}, \tilde{m}_{1}, \tilde{m}_{2} \rightarrow 0$ and choosing the helicity components we found the four-point amplitudes for gluons and massive scalars in four dimensions:

$$
\begin{aligned}
& \left.\mathcal{A}_{s}\left(1_{22}, 2_{11}, 3,4\right)\right|_{4 D}=-i \frac{\left\langle 1\left|\not \not_{3}^{(4)}\right| 2\right]}{s_{12} s_{23}}, \\
& \left.\mathcal{A}_{s}\left(1_{11}, 2_{11}, 3,4\right)\right|_{4 D}=i \mu^{2} \frac{[12]^{2}}{s_{12} s_{23}},
\end{aligned}
$$

where $\mu^{2}$ coincides in this case with the mass of the scalar squared.

Finally, the last amplitude one needs is the five-point tree-level amplitude. The amplitude with five-gluons has first been computed in [17]. In [14,62] this result has been extended to the five-point superamplitude in the $\mathcal{N}=$ $(1,1)$ theory. This superamplitude also contains information about the amplitude with scalar fields which is needed for the scalar subtraction when doing dimensional reconstruction. The amplitude with five gluons is

$$
\begin{aligned}
& \mathcal{A}_{g}\left(1_{a \dot{a}}, 2_{b \dot{b}}, 3_{c \dot{c}}, 4_{\dot{d}}, 5_{e \dot{e}}\right) \\
& \quad=\frac{i}{s_{12} s_{23} s_{34} s_{45} s_{51}}\left(-\mathcal{M}_{a \dot{a} b \dot{b} \dot{b} \dot{c} \dot{d} d \dot{e}}+\mathcal{D}_{a \dot{a} b \dot{b} c \dot{c} d \dot{d} e \dot{e}}\right)
\end{aligned}
$$

with

$$
\begin{aligned}
\mathcal{M}_{a \dot{a} b \dot{b} c \dot{c} d \dot{d} e \dot{e}}= & \left\langle 1_{a}\left|\not \not_{2} \not \not_{3} \not \phi_{4} \not \not_{5}\right| 1_{\dot{a}}\right]\left\langle 2_{b} 3_{c} 4_{d} 5_{e}\right\rangle\left[2_{\dot{b}} 3_{\dot{c}} 4 \dot{d} 5_{\dot{e}}\right] \\
& + \text { cyclic, }
\end{aligned}
$$

and

$$
\begin{aligned}
& 2 \mathcal{D}_{a \dot{a} b \dot{b} c \dot{c} d \dot{d} e \dot{e}}=\left\langle 1_{a} \tilde{\Sigma}_{2 \dot{b}}\right]\left\langle 2_{b} 3_{c} 4_{d} 5_{e}\right\rangle\left[1_{\dot{a}} 3_{\dot{c}} 4 \dot{d}_{\dot{d}} 5_{\dot{e}}\right]+\left\langle 3_{c} \tilde{\Sigma}_{4 \dot{d}}\right]\left\langle 1_{a} 2_{b} 4_{d} 5_{e}\right\rangle\left[1_{\dot{a}} 2_{\dot{b}} 3_{\dot{c}} 5_{\dot{e}}\right] \\
& +\left\langle 4_{d} \tilde{\Sigma}_{5 \dot{e}}\right]\left\langle 1_{a} 2_{b} 3_{c} 5_{e}\right\rangle\left[1_{\dot{a}} 2_{\dot{b}} 3_{\dot{c}} 4_{\dot{d}}\right]-\left\langle 3_{c} \tilde{\Sigma}_{5 \dot{e}}\right]\left\langle 1_{a} 2_{b} 4_{d} 5_{e}\right\rangle\left[1_{\dot{a}} 2_{\dot{b}} 3_{\dot{c}} 4_{\dot{d}}\right] \\
& -\left[1_{\dot{a}} \Sigma_{2 b}\right\rangle\left\langle 1_{a} 3_{c} 4_{d} 5_{e}\right\rangle\left[22_{\dot{b}} 3 \dot{c} 4{ }_{\dot{d}} 5_{\dot{e}}\right]-\left[3_{\dot{c}} \Sigma_{4 d}\right\rangle\left\langle 1_{a} 2_{b} 3_{c} 5_{e}\right\rangle\left[1_{\dot{a}} 2_{\dot{b}} 4{ }_{\dot{d}} 5_{\dot{e}}\right] \\
& -\left[4_{\dot{d}} \Sigma_{5 e}\right\rangle\left\langle 1_{a} 2_{b} 3_{c} 4_{d}\right\rangle\left[1_{\dot{a}} 2_{\dot{b}} 3_{\dot{c}} 5_{\dot{e}}\right]+\left[3_{\dot{c}} \tilde{\Sigma}_{5 e}\right\rangle\left\langle 1_{a} 2_{b} 3_{c} 4_{d}\right\rangle\left[1_{\dot{a}} 2_{\dot{b}} 4_{\dot{d}} 5_{\dot{e}}\right] .
\end{aligned}
$$

The amplitude with two scalars and three gluons is

$$
\mathcal{A}_{g}\left(1_{\phi}, 2_{\bar{\phi}}, 3_{c \dot{c}}, 4_{d \dot{d}}, 5_{e \dot{e}}\right)=-\frac{i}{s_{12} s_{23} s_{34} s_{45} s_{51}}\left(\mathcal{M}_{c \dot{c} d \dot{d} \dot{e} \dot{ }}^{s}+\mathcal{D}_{c \dot{c} d \dot{d} \dot{e} \dot{e}}^{s}\right)
$$

with

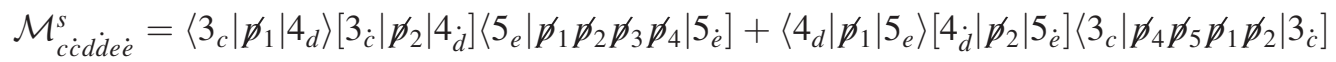

$$
\begin{aligned}
& +\left\langle 3_{c}\left|\not \not_{1}\right| 5_{e}\right\rangle\left[3_{\dot{c}}\left|\not \not_{2}\right| 5_{\dot{e}}\right]\left\langle 4_{d}\left|\not \not_{5} \not \not_{1} \not \not_{2} \not \not_{3}\right| 4_{\dot{d}}\right]+\frac{1}{2}\left\langle 3_{c} 4_{d} 5_{e} 1^{a}\right\rangle\left[3_{\dot{c}} 4_{\dot{d}} 5_{\dot{e}} 2_{\dot{b}}\right]\left\langle 1_{a} \tilde{\Sigma}_{2}^{\dot{b}}\right],
\end{aligned}
$$

and

$$
\begin{aligned}
& 2 \mathcal{D}_{c \dot{c} d \dot{d} e \dot{e}}^{s}=-\left\langle 4_{d}\left|\not \not_{1}\right| 5_{e}\right\rangle\left[3_{\dot{c}}\left|\not \not_{2}\right| 5_{\dot{e}}\right]\left\langle 3_{c} \tilde{\Sigma}_{4 \dot{d}}\right]+\left\langle 4_{d}\left|\not \not_{1}\right| 5_{e}\right\rangle\left[3_{\dot{c}}\left|\not \not_{2}\right| 4_{\dot{d}}\right]\left\langle 3_{c} \tilde{\Sigma}_{5 \dot{e}}\right] \\
& -\left\langle 3_{c}\left|\not \not_{1}\right| 5_{e}\right\rangle\left[3_{\dot{c}}\left|\not \not_{2}\right| 4_{\dot{d}}\right]\left\langle 4_{d} \tilde{\Sigma}_{5 \dot{e}}\right]+\left\langle 3_{c}\left|\not \not_{1}\right| 5_{e}\right\rangle\left[4_{\dot{d}}\left|\not \not_{2}\right| 5_{\dot{e}}\right]\left[3_{\dot{c}} \Sigma_{4 d}\right\rangle \\
& -\left\langle 3_{c}\left|\not \not_{1}\right| 4_{d}\right\rangle\left[4_{\dot{d}}\left|\not \not_{2}\right| 5_{\dot{e}}\right]\left[3_{\dot{c}} \Sigma_{5 e}\right\rangle+\left\langle 3_{c}\left|\not \not_{1}\right| 4_{d}\right\rangle\left[3_{\dot{c}}\left|\not \not_{2}\right| 5_{\dot{e}}\right]\left[4_{\dot{d}} \Sigma_{5 e}\right\rangle .
\end{aligned}
$$




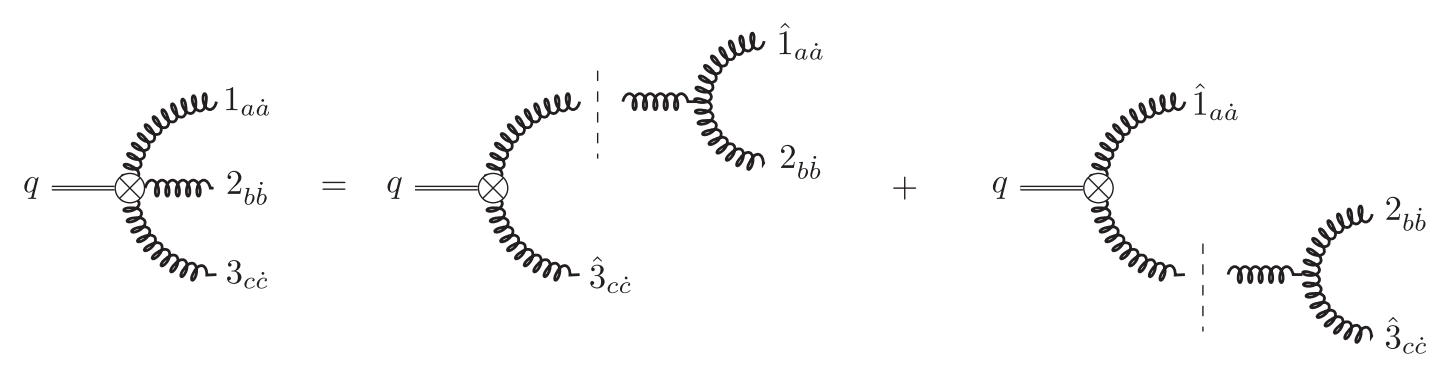

(a)

(b)

FIG. 8. BCFW construction of the tree-level nonminimal $\operatorname{Tr} F^{2}$ form factor in six dimensions.

The $\Sigma$ and $\tilde{\Sigma}$ that appear in the previous formulas are defined as

$$
\begin{aligned}
& \left|\Sigma_{i a}\right\rangle=\left(\not \not_{i} \not \not_{i+1} \not \not i_{i+2} \not \not_{i+3}-\not \not_{i} \not \not_{i+3} \not \not_{i+2} \not \not_{i+1}\right)\left|i_{a}\right\rangle
\end{aligned}
$$

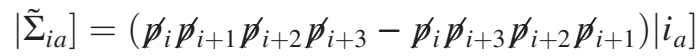

where we define $\not p_{6} \equiv \not p_{1}$.

\section{APPENDIX D: NONMINIMAL FORM FACTORS}

In this section we will address the computation of sixdimensional tree-level building blocks using BCFW recursion relations. ${ }^{21}$ In particular we briefly comment on the main steps of the calculation of $\operatorname{Tr} F^{2}$ in the nonminimal configuration.

Diagrammatically the terms we need to compute are represented in Fig. 8. In this computation one needs to make use of the three-point on-shell amplitudes in sixdimensions. These are most conveniently defined in terms of a set of auxiliary SU(2) spinors which we denote by $u_{a}, \tilde{u}_{\dot{a}}, w_{a}$ and $\tilde{w}_{\dot{a}}$, following the conventions of [17]. These objects are not Lorentz invariants in six dimensions and thus are not allowed to appear in the final expression, however they enjoy useful properties which simplify the calculation. The on-shell three-point amplitude cleanly expressed in terms of the above mentioned spinors:

$$
\mathcal{A}_{3}\left(1_{a \dot{a}}, 2_{b \dot{b}}, 3_{c \dot{c}}\right)=i \Gamma_{a b c}(1,2,3) \tilde{\Gamma}_{\dot{a} \dot{b} \dot{c}}(1,2,3),
$$

with

$$
\begin{aligned}
\Gamma_{a b c}(1,2,3) & =u_{1 a} u_{2 b} w_{3 c}+u_{1 a} w_{2 b} u_{3 c}+w_{1 a} u_{2 b} u_{3 c}, \\
\tilde{\Gamma}_{\dot{a} \dot{b} \dot{c}}(1,2,3) & =\tilde{u}_{1 \dot{a}} \tilde{u}_{2 \dot{b}} \tilde{w}_{3 \dot{c}}+\tilde{u}_{1 \dot{a}} \tilde{w}_{2 \dot{b}} \tilde{u}_{3 \dot{c}}+\tilde{w}_{1 \dot{a}} \tilde{u}_{2 \dot{b}} \tilde{u}_{3 \dot{c}} .
\end{aligned}
$$

\footnotetext{
${ }^{21}$ For a more detailed account of six-dimensional BCFW see [17].
}

Consider now applying six-dimensional BCFW as in Fig. 8. The hatted momenta are shifted by a quantity proportional to the complex parameter $z$ as

$$
\begin{aligned}
& \hat{p}_{1}=p_{1}+z X^{a \dot{a}} \varepsilon_{1 a \dot{a}}, \\
& \hat{p}_{3}=p_{3}-z X^{a \dot{a}} \varepsilon_{1 a \dot{a}},
\end{aligned}
$$

where $X^{a \dot{a}}$ is an arbitrary tensor needed to saturate the little group indices. This tensor, which also multiplies (D7), will be removed at the end of the calculation. The on-shell condition $\hat{p}_{1,2}^{2}=0$ implies $\operatorname{det} X=0$, which allows to express $X$ as

$$
X^{a \dot{a}}=x^{a} \tilde{x}^{\dot{a}} .
$$

Furthermore we can define the quantities

$$
y^{b}=\tilde{x}^{\dot{a}}\left\langle 3_{b} 1^{\dot{a}}\right]^{-1}, \quad \tilde{y}_{\dot{b}}=x^{a}\left\langle 1^{a} 3^{\dot{b}}\right]^{-1},
$$

which allow us to rewrite the momentum shift (D3) in terms of the spinor shifts

$$
\begin{aligned}
& \left|\hat{1}^{a}\right\rangle=\left|1^{a}\right\rangle+z x^{a} y_{b}\left|3^{b}\right\rangle, \\
& \left|\hat{3}^{b}\right\rangle=\left|3^{b}\right\rangle+z y^{b} x_{a}\left|1^{a}\right\rangle, \\
& \left.\left.\left.\mid \hat{1}^{\dot{a}}\right]=\mid 1^{\dot{a}}\right]-z \tilde{x}^{\dot{a}} \tilde{y}_{\dot{b}} \mid 3^{\dot{b}}\right], \\
& \left.\left.\left.\mid \hat{3}^{\dot{b}}\right]=\mid 3^{\dot{b}}\right]-z \tilde{y}^{\dot{b}} \tilde{x}_{\dot{a}} \mid 1^{\dot{a}}\right] .
\end{aligned}
$$

Considering now for example term A in Fig. 8 one has

$$
\begin{aligned}
(\mathrm{A}) & =X^{a \dot{a}} \mathcal{A}_{3}\left(\hat{1}_{a \dot{a}}, 2_{b \dot{b}}, \hat{k}_{d \dot{d}}\right) \frac{-i}{s_{12}} F_{\mathcal{O}_{2}}^{(0)}\left(-\hat{k}^{d \dot{d}}, \hat{3}_{c \dot{c}} ; q\right) \\
& =\frac{i}{s_{12}} X^{a \dot{a}} \Gamma_{a b d}(\hat{1}, 2, \hat{k}) \tilde{\Gamma}_{\dot{a} \dot{b} \dot{d}}(\hat{1}, 2, \hat{k})\left\langle\hat{k}^{d} \hat{3}_{\dot{c}}\right]\left\langle\hat{3}_{c} \hat{k}^{\dot{d}}\right]
\end{aligned}
$$

Before substituting the definitions (A6) in (D7), we make use of the properties of $u, \tilde{u}, w, \tilde{w}$, to simplify this expression. The most useful identities are 


$$
\begin{aligned}
& u_{i a} w_{i b}-u_{i b} w_{i a}=\epsilon_{a b}, \quad \tilde{u}_{i \dot{a}} \tilde{w}_{i \dot{b}}-\tilde{u}_{i b} \tilde{w}_{i \dot{a}}=\epsilon_{\dot{a} \dot{b}}, \\
& \left.\left.\left|u_{i} \cdot i\right\rangle=\left|u_{j} \cdot j\right\rangle, \quad \mid \tilde{u}_{i} \cdot i\right]=\mid \tilde{u}_{j} \cdot j\right] \quad \forall i, j=1,2, k, \\
& \left|w_{1} \cdot 1\right\rangle+\left|w_{2} \cdot 2\right\rangle+\left|w_{k} \cdot k\right\rangle=0, \\
& \left.\left.\left.\mid \tilde{w}_{1} \cdot 1\right]+\mid \tilde{w}_{2} \cdot 2\right]+\mid \tilde{w}_{k} \cdot k\right]=0,
\end{aligned}
$$

where we used the shorthand notation $u_{i a}\left|i^{a}\right\rangle=\left|u_{i} \cdot i\right\rangle$ and $\left.\left.\tilde{u}_{i \dot{a}} \mid i^{\dot{a}}\right]=\mid \tilde{u}_{i} \cdot i\right]$. These identities allow us to rewrite

$$
\begin{aligned}
& \Gamma_{a b d}(\hat{1}, 2, \hat{k})\left\langle\hat{k}^{d}\right|=\left\langle\hat{1}_{a}\right| u_{2 b}+\left\langle 2_{b}\right| u_{\hat{1} a}, \\
& \left.\left.\left.\tilde{\Gamma}_{\dot{a} \dot{b} \dot{d}}(\hat{1}, 2, \hat{k}) \mid \hat{k}^{\dot{d}}\right]=\mid \hat{1}_{\dot{a}}\right] \tilde{u}_{2 \dot{b}}+\mid 2_{\dot{b}}\right] \tilde{u}_{\hat{1} \dot{a}},
\end{aligned}
$$

which in turn leads to

$$
\begin{aligned}
(\mathrm{A})= & \frac{i}{s_{12}} X^{a \dot{a}}\left(\left\langle\hat{1}_{a} \hat{3}_{\dot{c}}\right]\left\langle\hat{3}_{c} \hat{1}_{\dot{a}}\right] u_{2 b} \tilde{u}_{2 \dot{b}}+\left\langle\hat{1}_{a} \hat{3}_{\dot{c}}\right]\left\langle\hat{3}_{c} 2_{\dot{b}}\right] u_{2 b} \tilde{u}_{1 \dot{a}}\right. \\
& \left.+\left\langle 2_{b} \hat{3}_{\dot{c}}\right]\left\langle\hat{3}_{c} \hat{1}_{\dot{a}}\right] u_{\hat{1} a} \tilde{u}_{2 \dot{b}}+\left\langle 2_{b} \hat{3}_{\dot{c}}\right]\left\langle\hat{3}_{c} 2_{\dot{b}}\right] u_{\hat{1} a} \tilde{u}_{\hat{1} \dot{a}}\right) .
\end{aligned}
$$

To further simplify the result, and to eliminate the residual $\mathrm{SU}(2)$ spinors, we make the following observations:

(i) pairs of $u_{i}, \tilde{u}_{j}$ with $i \neq j$ can be immediately rewritten in terms of six-dimensional invariants as

$$
\begin{array}{ll}
u_{1 a} \tilde{u}_{2 \dot{b}}=\left\langle 1_{a} 2_{\dot{b}}\right], & u_{2 b} \tilde{u}_{1 \dot{a}}=-\left\langle 2_{b} 1_{\dot{a}}\right], \\
u_{2 b} \tilde{u}_{k \dot{c}}=\left\langle 2_{b} k_{\dot{c}}\right], & u_{k c} \tilde{u}_{2 \dot{b}}=-\left\langle k_{c} 2_{\dot{b}}\right] .
\end{array}
$$

(ii) pairs of $u_{i}, \tilde{u}_{j}$ with $i=j$ can be rewritten using the identity [62]

$$
u_{i a} \tilde{u}_{i \dot{a}}=\frac{(-1)^{\mathcal{P}_{i j}}}{s_{i P}}\left\langle i_{a}\left|p_{j} P\right| i_{\dot{a}}\right]
$$

where $p_{j}$ is any other momentum belonging to the same three-point amplitude as $p_{i}$, and $\mathcal{P}_{i j}=+1$ for clockwise ordering of the states $(i, j)$. Also $P$ is any given arbitrary momentum.

Repeating a similar reasoning on term (B) one gets

$$
\begin{aligned}
(\mathrm{B})= & \frac{i}{s_{23}} X^{a \dot{a}}\left(\left\langle\hat{1}_{a} \hat{3}_{\dot{c}}\right]\left\langle\hat{3}_{c} \hat{1}_{\dot{a}}\right] u_{2 b} \tilde{u}_{2 \dot{b}}+\left\langle\hat{1}_{a} \hat{3}_{\dot{c}}\right]\left\langle 2_{b} \hat{1}_{\dot{a}}\right] u_{3 c} \tilde{u}_{2 \dot{b}}\right. \\
& \left.+\left\langle\hat{1}_{a} 2_{\dot{b}}\right]\left\langle\hat{3}_{c} \hat{1}_{\dot{a}}\right] u_{2 b} \tilde{u}_{\hat{3} \dot{c}}+\left\langle\hat{1}_{a} 2_{\dot{b}}\right]\left\langle 2_{b} \hat{1}_{\dot{a}}\right] u_{\hat{\beta}_{c} \tilde{u}_{\hat{3} \dot{c}}}\right) .
\end{aligned}
$$

The on-shell condition for the intermediate propagators in (A) and (B) defines two different BCFW shift

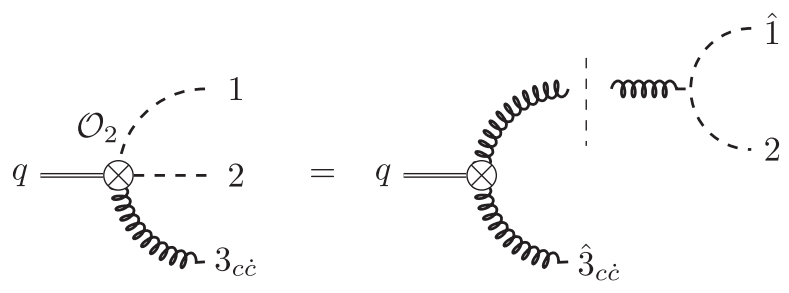

FIG. 9. BCFW construction of the tree-level nonminimal $\operatorname{Tr} F^{2}$ form factor with two scalars.

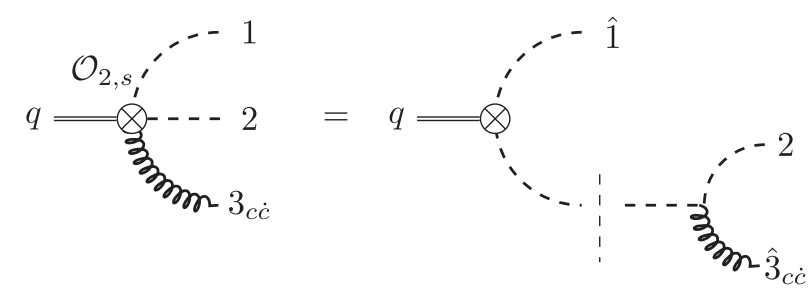

FIG. 10. BCFW construction of the tree-level nonminimal $D \phi^{2}$ form factor.

parameters, which we label $z_{A}$ and $z_{B}$ respectively. By computing $z_{A}$ and $z_{B}$ one can see that they are related by

$$
z_{B}=-\frac{s_{23}}{s_{12}} z_{A}
$$

Thanks to this relation multiple cancellations happen between terms in (A) and terms in (B). With some further algebra and removing the $X^{a \dot{a}}$ tensor, one arrives at (3.13).

The analytic expression of the six-dimensional form factor $F_{\mathcal{O}_{2}}^{(0)}\left(1_{a \dot{a}}, 2_{b \dot{b}}, 3_{c \dot{c}} ; q\right)$ could also be computed using Feynman diagrams, see for example [16]. Due to the low multiplicity of this form factor, there is just a small number of contributing Feynman diagrams. The diagrammatic approach may thus be considered as equivalently viable as BCFW in this case, the latter method however leads to a far more compact expression with all the symmetries manifest.

In a similar way but with much less involved calculation, we can find both the nonminimal form factors with two scalars and one gluon (3.14) and (3.15). In Figs. 9 and 10 we show the BCFW factorization channels for these calculations. The only missing ingredient is the three-point amplitude with two scalars and one gluon in six dimensions, which turns out to be very simple:

$$
\mathcal{A}\left(1_{a \dot{a}}, 2,3\right)=i u_{1 a} \tilde{u}_{1 \dot{a}} .
$$




\section{APPENDIX E: INTEGRAL EXPRESSIONS}

The integrals needed in this paper are
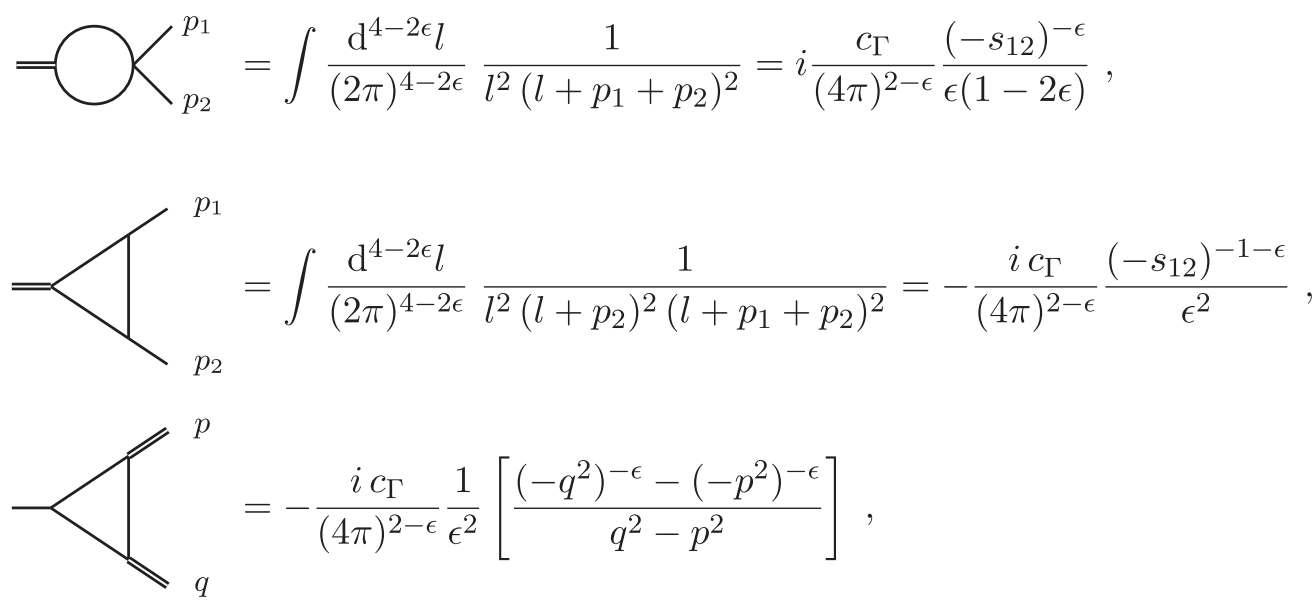

and

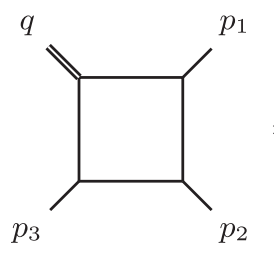

$$
\begin{aligned}
= & \int \frac{\mathrm{d}^{4-2 \epsilon} l}{(2 \pi)^{4-2 \epsilon}} \frac{1}{l^{2}\left(l+p_{1}\right)^{2}\left(l+p_{1}+p_{2}\right)^{2}\left(l+p_{1}+p_{2}+p_{3}\right)^{2}} \\
= & -\frac{2 i c_{\Gamma}}{(4 \pi)^{2-\epsilon}} \frac{1}{s_{12} s_{23}}\left\{-\frac{1}{\epsilon^{2}}\left[\left(-s_{12}\right)^{-\epsilon}+\left(-s_{23}\right)^{-\epsilon}-\left(-q^{2}\right)^{-\epsilon}\right]+\right. \\
& \left.+\operatorname{Li}_{2}\left(1-\frac{s_{12}}{q^{2}}\right)+\operatorname{Li}_{2}\left(1-\frac{s_{23}}{q^{2}}\right)+\frac{1}{2} \log ^{2}\left(\frac{s_{12}}{s_{23}}\right)+\frac{\pi^{2}}{6}\right\}+\mathcal{O}(\epsilon),
\end{aligned}
$$

where

$$
c_{\Gamma}=\frac{\Gamma[1+\epsilon] \Gamma[1-\epsilon]^{2}}{\Gamma[1-2 \epsilon]} .
$$

This results are exact to all orders in $\epsilon$, and the expression of the corresponding integral functions in a different number of dimensions can be obtained by simply replacing $\epsilon$ to the appropriate value, for instance $\epsilon \mapsto \epsilon-1$ and $\epsilon \mapsto \epsilon-2$ for $d=6-2 \epsilon$ and $d=8-2 \epsilon$, respectively. In particular it turns out that all the integrals which give the rational terms, i.e., those with a nontrivial numerator written in (2.4), can always be expressed as integrals in higher dimensions [8]. Indeed consider the general integral function

$$
\begin{aligned}
I_{n}^{d}\left[\mu^{2 p}\right] & =\int \frac{\mathrm{d}^{4-2 \epsilon} l}{(2 \pi)^{4-2 \epsilon}}\left(\mu^{2}\right)^{p} f_{n}\left(\left\{p_{i}\right\}, l\right) \\
& =\int \frac{\mathrm{d}^{4} l^{(4)}}{(2 \pi)^{4}} \int \frac{\mathrm{d}^{-2 \epsilon} \mu}{(2 \pi)^{-2 \epsilon}}\left(\mu^{2}\right)^{p} f_{n}\left(\left\{p_{i}\right\}, l\right),
\end{aligned}
$$

and the $\mu$-measure can be rewritten as

$$
\begin{aligned}
\int \mathrm{d}^{-2 \epsilon} \mu\left(\mu^{2}\right)^{p} & =\frac{1}{2} \int \mathrm{d} \Omega_{-1-2 \epsilon} \int_{0}^{+\infty} \mathrm{d} \mu^{2}\left(\mu^{2}\right)^{-1-\epsilon+p} \\
& =\frac{\int \Omega_{-1-2 \epsilon}}{\int \mathrm{d} \Omega_{2 p-1-2 \epsilon}} \int \mathrm{d}^{2 p-2 \epsilon} \mu .
\end{aligned}
$$

Then (E4) can be written as

$$
\begin{aligned}
I_{n}^{d}\left[\mu^{2 p}\right] & =\frac{(2 \pi)^{2 p} \int \mathrm{d} \Omega_{-1-2 \epsilon}}{\int \mathrm{d} \Omega_{2 p-1-2 \epsilon}} \int \frac{\mathrm{d}^{4+2 p-2 \epsilon} l}{(2 \pi)^{4+2 p-2 \epsilon}} f_{n}\left(\left\{p_{i}\right\}, l\right) \\
& =-\epsilon(1-\epsilon)(2-\epsilon) \cdots(p-1-\epsilon)(4 \pi)^{p} I_{n}^{d+2 p}[1],
\end{aligned}
$$

where

$$
\int \mathrm{d} \Omega_{x}=\frac{2 \pi^{\frac{x+1}{2}}}{\Gamma\left[\frac{x+1}{2}\right]} .
$$

Then to compute this integrals becomes just simple algebra: 

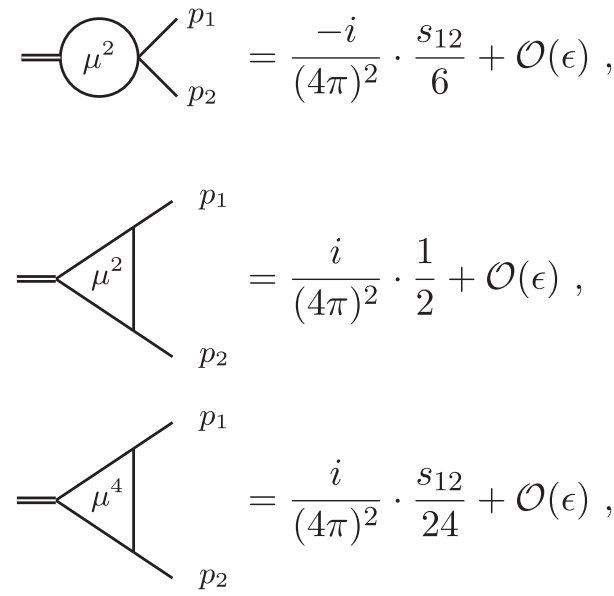

\section{APPENDIX F: THE SpinorHelicity6D Mathematica PACKAGE}

In this section we briefly describe the Mathematica package spinorHelicity6D [83], which we developed to facilitate our calculations involving six- (and four-) dimensional spinors and was initially inspired by the package spinorHelicity [58]. In the following we present a subset of all the available functions-specifically, we focus on the routines needed to check our results. In the near future a more complete documentation along with an updated version of the package will be released, including tools needed for numerical evaluations in sixdimensional space. For the sake of concreteness, the functions will be presented applying them to an example calculation, namely $\operatorname{Tr} F^{3}$ in the all-plus helicity configuration $^{22}$.

\section{The Building Blocks}

The basic input of most computations is given in terms of spinors and invariants built out of them. All of these can be input into Mathematica through a keyboard shortcut, a complete list of which is stored into the variable SHORTCUTS, see Fig. 11. Alternatively one can use the more user-friendly palette which opens automatically upon loading the package or by typing spinorPalette.

The basic objects are:

(i) the standard four-dimensional spinors $\lambda$ and $\tilde{\lambda}$. These allow for upper and lower spinor-indices and have the usual contraction properties:

\footnotetext{
${ }^{22}$ See also the Mathematica notebook in the Github repository.
}

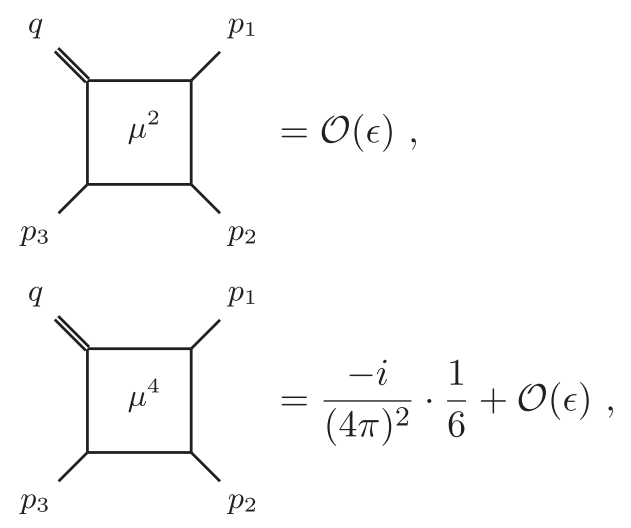

$$
\begin{aligned}
& \ln [3]:=\lambda^{\mathrm{a}}[1] \lambda_{\mathrm{a}}[2] \\
& \text { Out[3] }=\left\langle\begin{array}{ll}
1 & 2
\end{array}\right\rangle \\
& \ln [4]:=\epsilon^{a b} \lambda_{a}[1] \lambda_{b}[2] \\
& \mathrm{Out}[4]=-\left\langle\begin{array}{ll}
1 & 2
\end{array}\right\rangle \\
& \ln [5]:=\tilde{\lambda}_{\mathrm{a}}[1] \tilde{\lambda}^{\mathrm{a}}[2] \\
& \text { Out[5] }=\left[\begin{array}{ll}
1 & 2
\end{array}\right]
\end{aligned}
$$

The indices of $\lambda$ and $\tilde{\lambda}$ do not mix, in other words the package is able to distinguish between dotted and undotted indices:

$$
\begin{aligned}
& \operatorname{In}[6]:=\lambda^{\mathrm{a}}[1] \tilde{\lambda}_{\mathrm{a}}[2] \lambda_{\mathrm{a}}[3] \tilde{\lambda}^{\mathrm{a}}[4] \\
& \text { Out[6]=}\left\langle\begin{array}{lll}
1 & 3\rangle
\end{array}\right]\left[\begin{array}{ll}
2 & 4
\end{array}\right]
\end{aligned}
$$

We adopted the convention that

$$
\lambda(-p)=i \lambda(p), \quad \tilde{\lambda}(-p)=i \tilde{\lambda}(p),
$$

and this is automatically applied both to the free as well as contracted spinors:

$$
\begin{aligned}
& \ln [8]:=\lambda^{\mathrm{a}}[-\mathbf{1}] \\
& \text { Out[8]=} \dot{\mathbb{1}} \lambda^{\mathrm{a}}[1] \\
& \ln [9]:=[1-\text { test }] \\
& \text { Out[9]= } \dot{\mathbb{1}}[1 \text { test }]
\end{aligned}
$$

As can be seen from the above example one is free to choose whatever label for the momenta. However some 


\section{$\ln [2]:=$ \$Shortcuts}

Out[2]/MatrixForm=

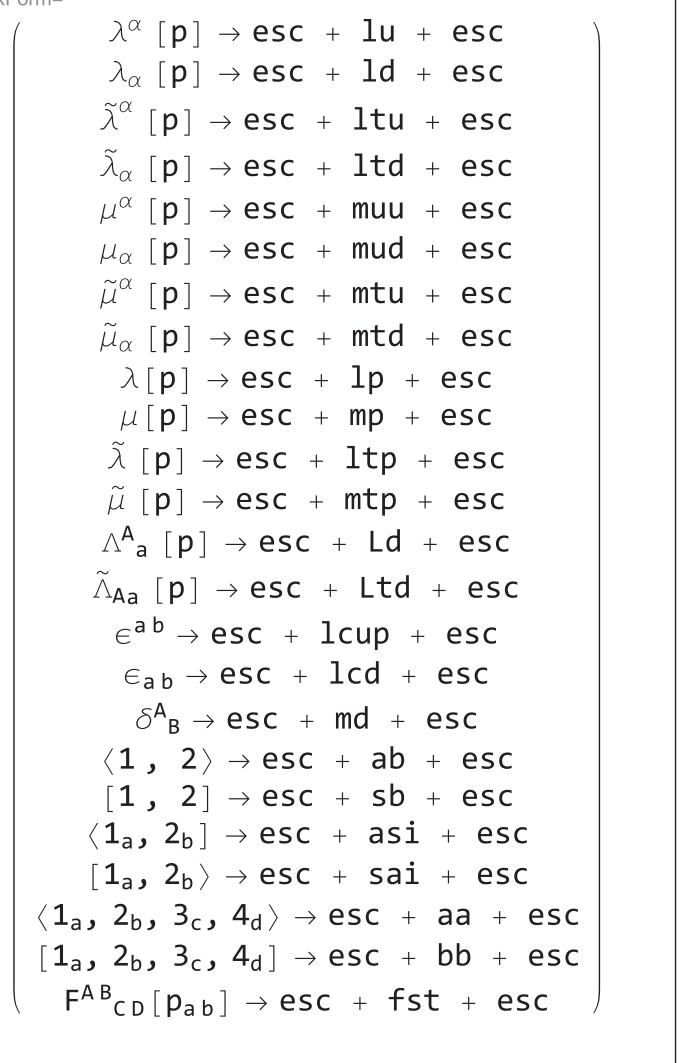

FIG. 11. A complete list of all the shortcuts available in the package.

caution is needed and we recommend avoiding labels containing minus signs, ${ }^{23}$ these are safe only if written in form of a string.

(ii) $\mu$ and $\tilde{\mu}$ are the auxiliary spinors needed to write a massive momentum in terms of two massless ones as in (B23). They share all the properties of $\lambda$ and $\tilde{\lambda}$ respectively.

(iii) The set of spinors $\lambda$ and $\mu$ without indices are auxiliary objects used for example when defining properties or applying transformations to both spinors with upper and lower Lorentz indices.

(iv) M and $\tilde{M}$ are the "masses" arising from the fifth and sixth spacetime component of the momentum as in (4.5).

(v) $\Lambda$ and $\tilde{\Lambda}$ are the six-dimensional spinors. These have been implemented only with lower little group indices, but in order to raise them one can simply use the two-dimensional Levi-Civita tensor.

(vi) Clearly $\langle\cdot, \cdot\rangle$ and $[\cdot, \cdot]$ are the four-dimensional invariants and $\langle\cdot, \cdot],[\cdot, \cdot\rangle,\langle\cdot, \cdot, \cdot, \cdot\rangle$ and $[\cdot, \cdot, \cdot, \cdot]$ the six-dimensional ones.

\footnotetext{
${ }^{23}$ For example $n-1$ could be misinterpreted as the opposite of $1-n$ due to the automatic ordering applied by Mathematica.
}

It is important to point out that, despite the nice visualization properties of the above presented objects, they are still interpreted as plain functions by Mathematica. This means that, for example, they can be safely copy-pasted and all the standard operations for functions can be applied. In order to access the explicit functional forms use InputForm.

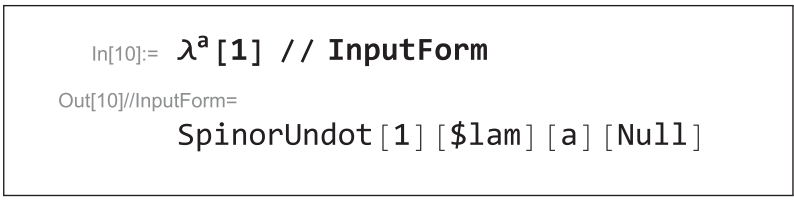

\section{Computing the Double-Cut}

The computation of $\operatorname{Tr} F^{3}$ (all-plus) begins by imposing four-dimensional kinematics on external particles, or equivalently declaring the corresponding momenta to be massless. This is done through the function KillMasses, and the list of massless momenta ${ }^{24}$ is stored in Momenta4D

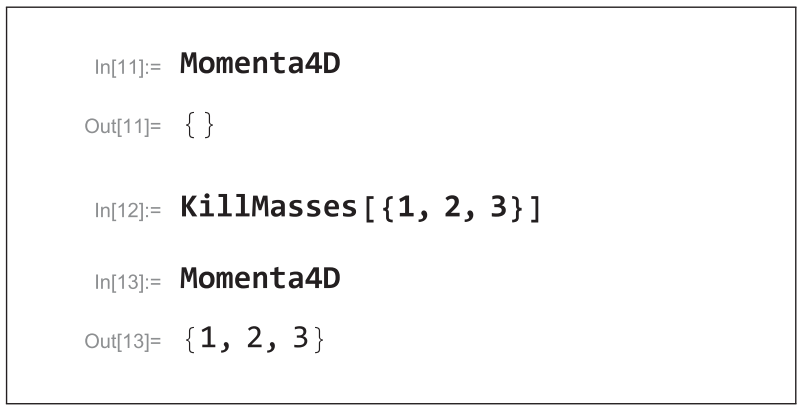

In our specific case we need to break down the sixdimensional spinors and invariants in terms of the fourdimensional ones. This breakdown is achieved through the command TO4D locally on a given expression, but it can also be turned on globally and applied automatically to all expressions through To4DAlways [True]. This setting can be reversed by To4DAlways [False].

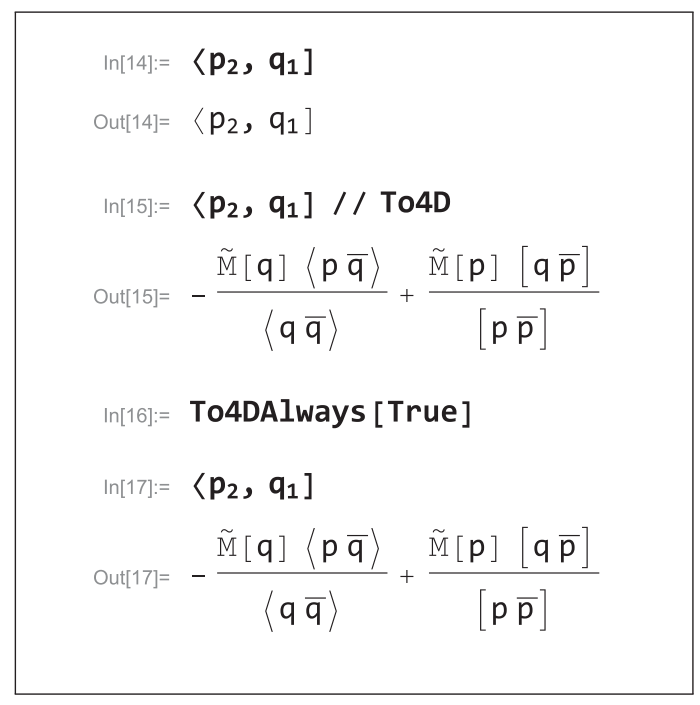

\footnotetext{
${ }^{24} \mathrm{By}$ default all momenta are always considered massive.
} 
One can then write down the following double-cut expression with gluons running through the loop:

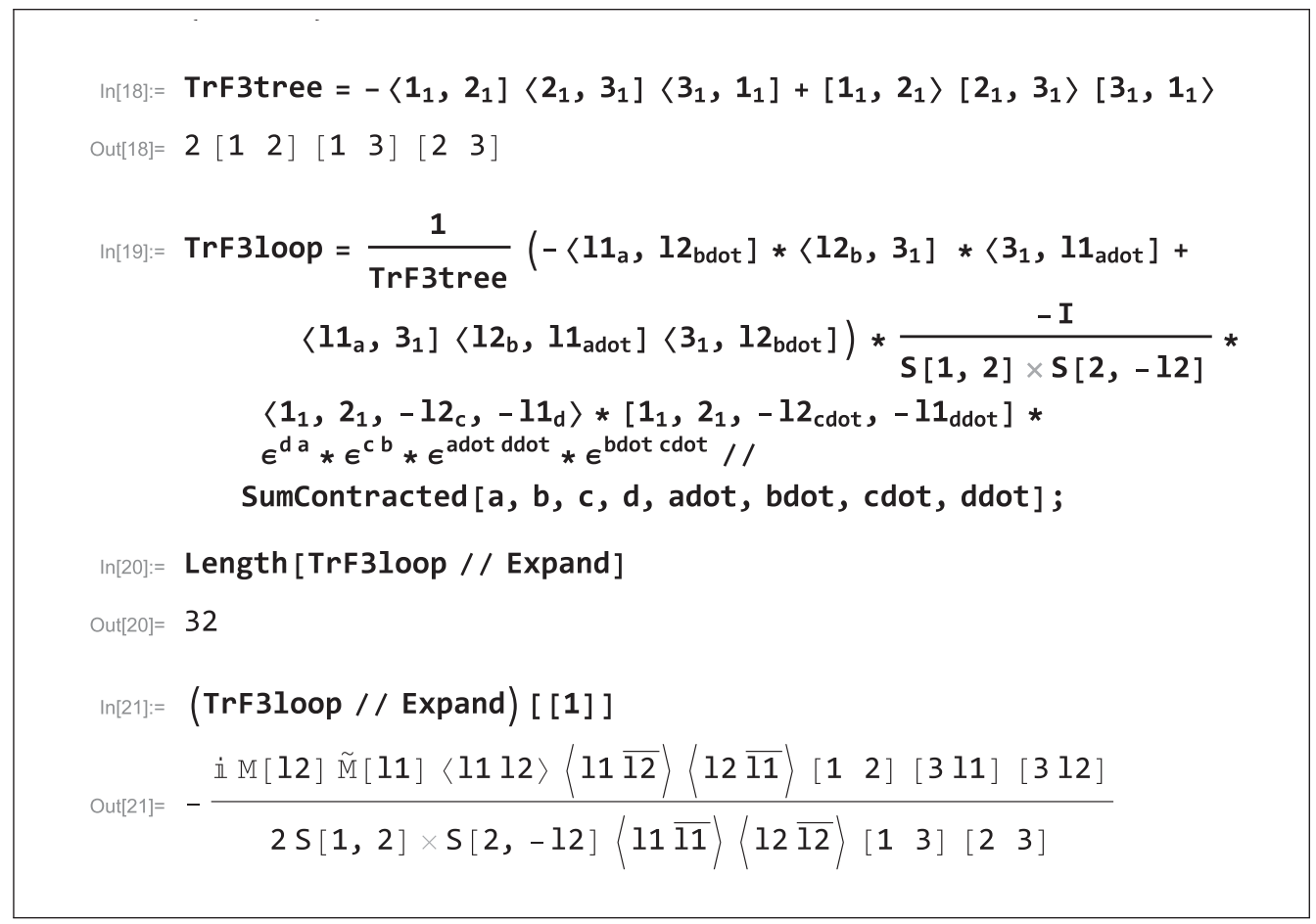

Here TrF3tree is the tree-level form factor used to normalize the one-loop expression, and $S$ is the Mandelstam invariant $s_{i j}$ with momenta $i$ and $j$ in six-dimensions. We made use of the command SumContracted, which sums over the contracted little-group indices. As can be seen the result of the double cut is a 32-term expression, and we printed the first term of the sum to give an idea of their form. We can obtain a first simplification of the expression by applying momentum conservation in the form of (4.5), which reduces the number of terms down to 24 :

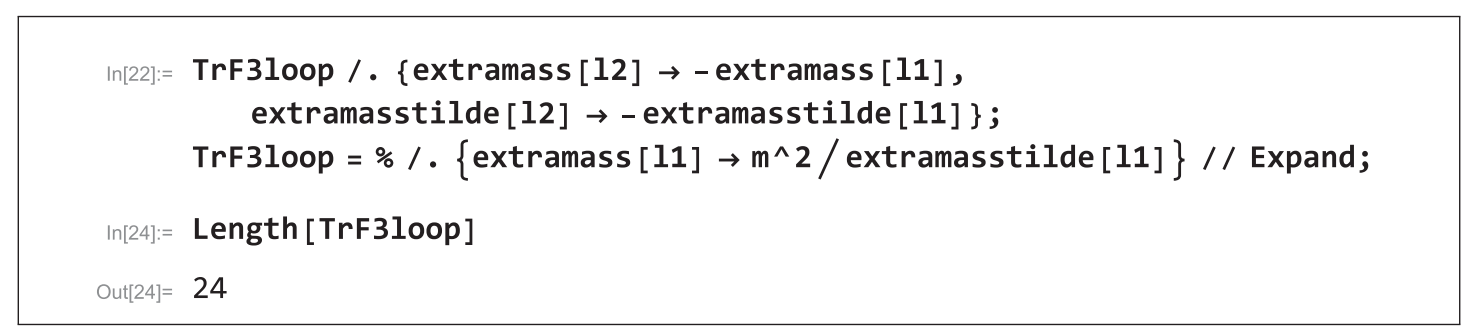

\section{Removing the redundancy}

Now we can use the function MuReplace to eliminate the redundant d.o.f. parametrized by the $\{\mu, \tilde{\mu}\}$ spinors. These can be fixed to arbitrary values without affecting the final (little-group invariant) result. MuReplace goes through all the $\mu \mathrm{s}(\tilde{\mu})$ present in the given expression and replaces them with $\lambda \mathrm{s}(\tilde{\lambda})$ in such a way that as many terms as possible vanish due to the antisymmetry of the angle and square brackets. This function allows for two options:

(i) DisplayReplacements, default is False. If set to True the replacements chosen by MuReplace will be displayed along with the result. (ii) GlobalReplacements, default is False. If set to True the chosen replacements are stored and become globally defined. ${ }^{25}$ The list of spinors defined to be equivalent can be accessed through Fixedspinors. The global definitions stored in FixedSpinors can be cleared with Clearspinors.

\footnotetext{
${ }^{25}$ In other words the chosen replacements are applied from there on whenever the given spinors are encountered.
} 


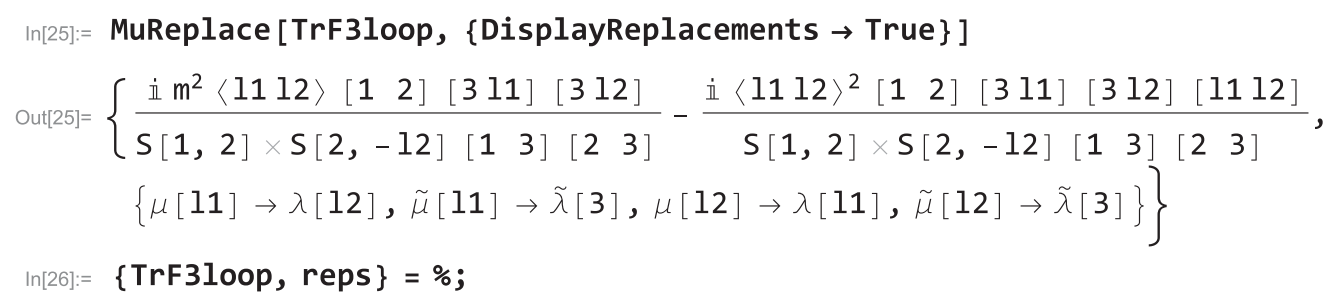

The output of MuReplace upon setting DisplayReplacements to True is a list of two elements. The first is the expression of $\operatorname{Tr} F^{3}$ after the most suitable replacements have been applied. The second term is the list of replacements which have been found to be the most convenient.

It is also possible for the user to choose the replacements and apply them manually. This can be done through the function SpinorReplace or SpinorReplaceSequential, which allow to perform generic replacements of spinors inside given expressions. The difference between the two functions is that the first performs all the specified replacements simultaneously whereas the second performs them sequentially.

Finally, before performing any other manipulations, we restore the momenta $l_{1}$ and $l_{2}$ to massive four-dimensional momenta, as discussed in more detail in Sec. IV B. This is done by the function CompleteToMassive, which takes as input an expression and the list of replacements used to remove the $\mu$ s. One gets

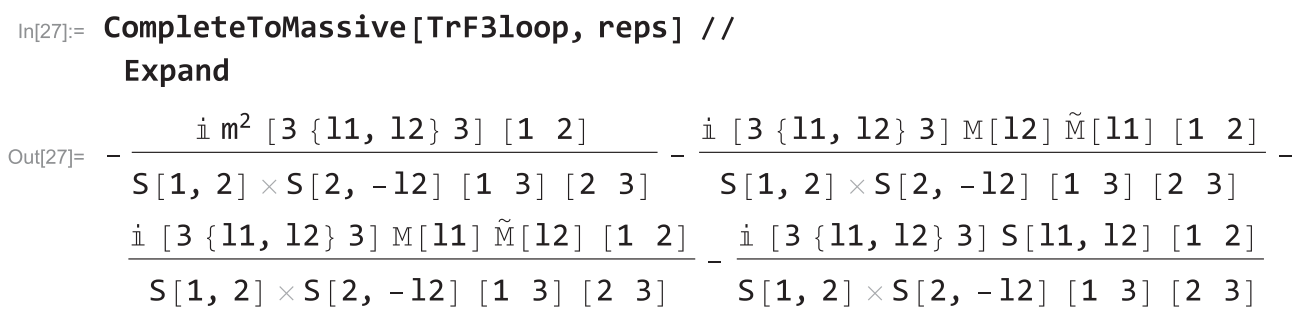

where the spinor invariants $\left[3 l_{1}\right]\left\langle l_{1} l_{2}\right\rangle\left[l_{2} 3\right]$ has been closed to $\left[3 l_{1} l_{2} 3\right]$ and new dependencies on the masses $m$ and $\tilde{m}$ appeared. Using once again momentum conservation to get rid of $m_{l_{2}}$ and $\tilde{m}_{l_{2}}$, and to replace $s_{l_{1} l_{2}}$ with $s_{12}$ we get

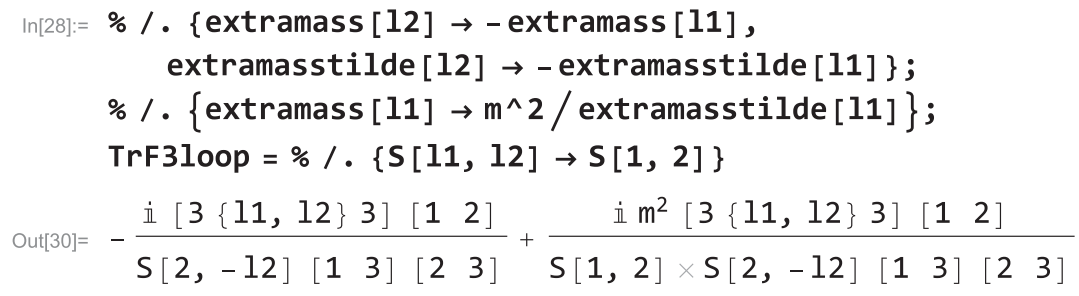

\section{The scalar subtraction}

Once the computation with the gluons in the loop has been completed we can move on to the scalars. One has to go through exactly the same steps as before. First write down the double-cut expression, then evaluate it and remove the redundant $\mu$ spinors to simplify the result, and finally rewrite the expression in terms of four-dimensional massive momenta. 


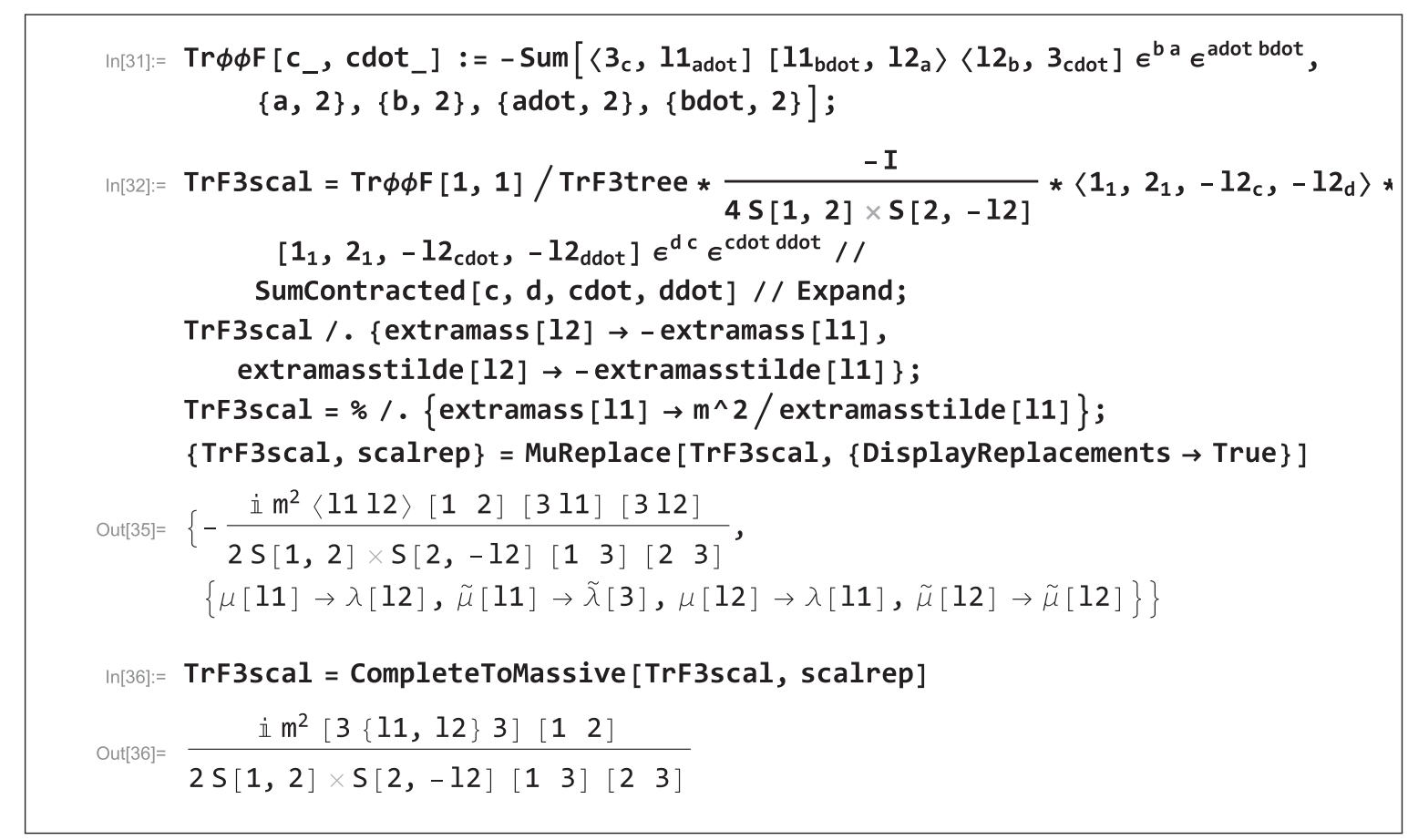

Performing the scalar subtraction leads then to the final result:

$$
\begin{aligned}
& \operatorname{In}[37]:=\text { int }=\operatorname{TrF3loop}-2 * \operatorname{TrF3scal} \\
& \text { Out[37]=}=-\frac{\dot{\mathbb{1}}\left[3\left\{\begin{array}{ll}
11,12\} & 3
\end{array}\right]\left[\begin{array}{ll}
1 & 2
\end{array}\right]\right.}{\mathrm{S}[2,-12]\left[\begin{array}{ll}
1 & 3
\end{array}\right]\left[\begin{array}{ll}
2 & 3
\end{array}\right]}
\end{aligned}
$$

From here on one can take two possible routes: either manipulate the final expression by hand and obtain (4.32), where the IR divergences are clearly visible in the form of a one-mass triangle. If one is interested in a completely reduced expression, one can proceed as follows:

(i) complete the denominator to Mandelstam invariants and further contract all possible expressions of the form $\langle i j\rangle[j i]$ to $s_{i j}$. This is accomplished with CompleteDenomina-tors and CompleteMandelstam respectively. The variables $\mathrm{S} 4$ is the Mandelstam invariant $s_{i j}$ with $p_{i}$ and $p_{j}$ in four dimensions, where $\mathrm{S} 4$ can be related to $\mathrm{S}$ through CompleteToMassive.

$$
\begin{aligned}
& \ln [38]:=\text { int }=\text { int // CompleteDenominators // CompleteMandelstam }
\end{aligned}
$$

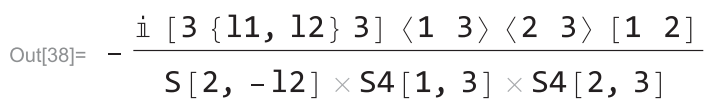

(ii) Since we removed the helicity structure, int must be expressible in terms of Mandelstam invariants only. In other words we expect the numerator to be of the form $\left\langle i \not p_{j} \cdots \not p_{k} i\right]$, which is a trace involving a helicity projector. To contract the numerator into the above form we use ToChain:

$$
\begin{aligned}
& \operatorname{In}[39]:=\text { ToChain [int }] \\
& \text { Out[39] }=-\frac{\dot{\mathbb{1}}\langle 2\{3,12,11,3,1\} 2]}{\mathrm{S}[2,-12] \times \mathrm{S} 4[1,3] \times \mathrm{S} 4[2,3]}
\end{aligned}
$$


and then we use ToTrace to evaluate the resulting trace. The option KillEpsilon sets the contributions proportional to the Levi-Civita tensor to zero. We can do this since the kinematics of the problem does not allow for more than three independent momenta in the final answer and thus similar terms would be vanishing anyway. The scalar products appearing after the trace are four dimensional.

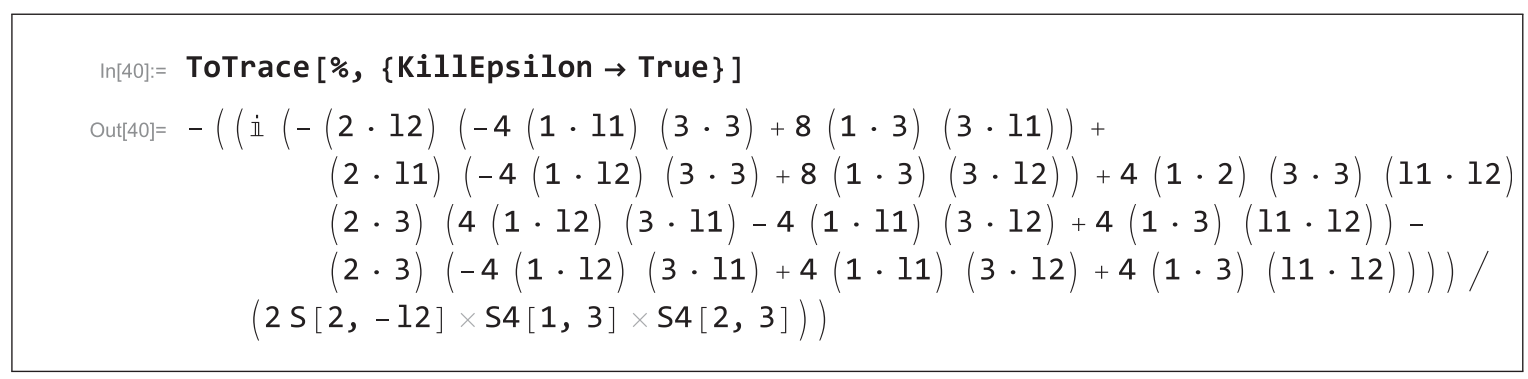

(iii) rewrite the four-dimensional scalar products as six-dimensional Mandelstam invariants and masses, using ScalProdTos

$$
\begin{aligned}
\text { Out[41]= } & -\frac{\text { i } M[12] \tilde{M}[11] \mathrm{S}[1,2] \times \mathrm{S}[3,3]}{4 \mathrm{~S}[2,-12] \times \mathrm{S} 4[1,3] \times \mathrm{S} 4[2,3]}- \\
& \frac{\text { i } \mathrm{M}[11] \tilde{\mathrm{M}}[12] \mathrm{S}[1,2] \times \mathrm{S}[3,3]}{4 \mathrm{~S}[2,-12] \times \mathrm{S} 4[1,3] \times \mathrm{S} 4[2,3]}+\frac{\text { i } \mathrm{S}[1,12] \times \mathrm{S}[2,11] \times \mathrm{S}[3,3]}{4 \mathrm{~S}[2,-12] \times \mathrm{S} 4[1,3] \times \mathrm{S} 4[2,3]}- \\
& \frac{\dot{ } \mathrm{S}[1,11] \times \mathrm{S}[2,12] \times \mathrm{S}[3,3]}{4 \mathrm{~S}[2,-12] \times \mathrm{S} 4[1,3] \times \mathrm{S} 4[2,3]}-\frac{\text { i } \mathrm{S}[1,12] \times \mathrm{S}[2,3] \times \mathrm{S}[3,11]}{2 \mathrm{~S}[2,-12] \times \mathrm{S} 4[1,3] \times \mathrm{S} 4[2,3]} \\
& \frac{\text { i } \mathrm{S}[1,3] \times \mathrm{S}[2,12] \times \mathrm{S}[3,11]}{2 \mathrm{~S}[2,-12] \times \mathrm{S} 4[1,3] \times \mathrm{S} 4[2,3]}+\frac{\text { i } \mathrm{S}[1,11] \times \mathrm{S}[2,3] \times \mathrm{S}[3,12]}{2 \mathrm{~S}[2,-12] \times \mathrm{S} 4[1,3] \times \mathrm{S} 4[2,3]} \\
& \frac{\text { i } \mathrm{S}[1,3] \times \mathrm{S}[2,11] \times \mathrm{S}[3,12]}{2 \mathrm{~S}[2,-12] \times \mathrm{S} 4[1,3] \times \mathrm{S} 4[2,3]}-\frac{\text { i } \mathrm{S}[1,2] \times \mathrm{S}[3,3] \times \mathrm{S}[11,12]}{4 \mathrm{~S}[2,-12] \times \mathrm{S} 4[1,3] \times \mathrm{S} 4[2,3]}
\end{aligned}
$$

(iv) then we use momentum conservation once again, and since all the $S 4$ appearing in the expression involve only external four-dimensional momenta, we uplift it to $S$.

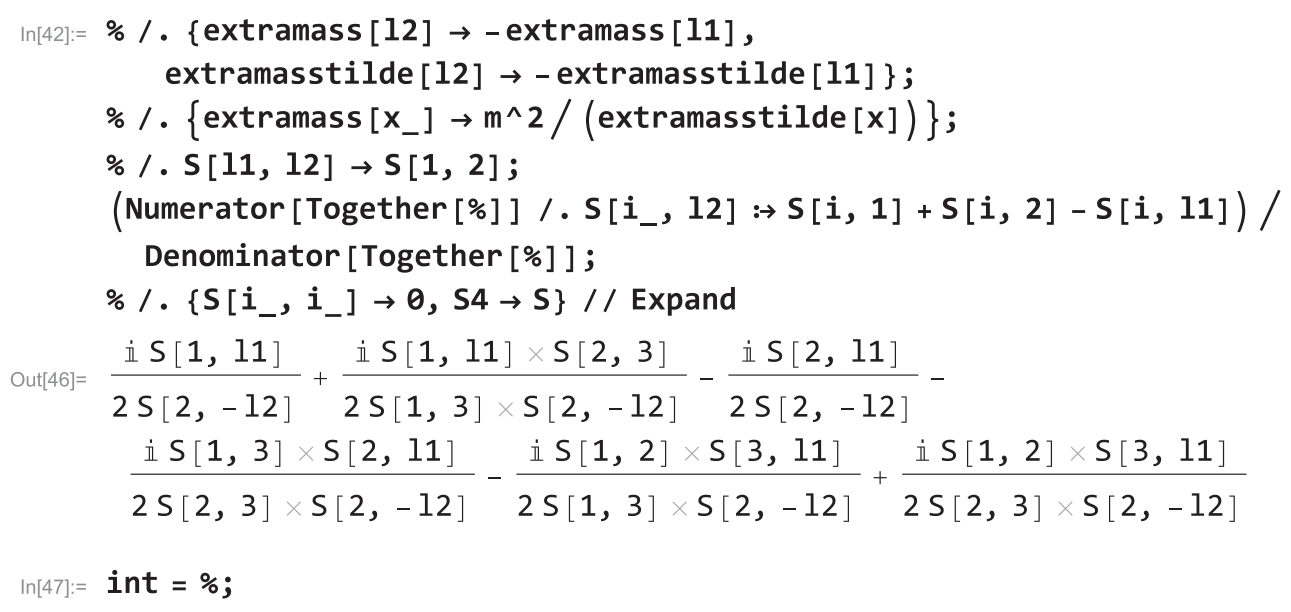


(v) Finally we can perform the reduction of the above expression using integration by part identities, for example with LiteRed $[84,85]$. We export our result to the LiteRed notation using the function Relabel, which allows to relabel the momenta inside the scalar products and Mandelstam invariants, as well as reassign a new name to the scalar product. We also uplift the cut and then perform the reduction:

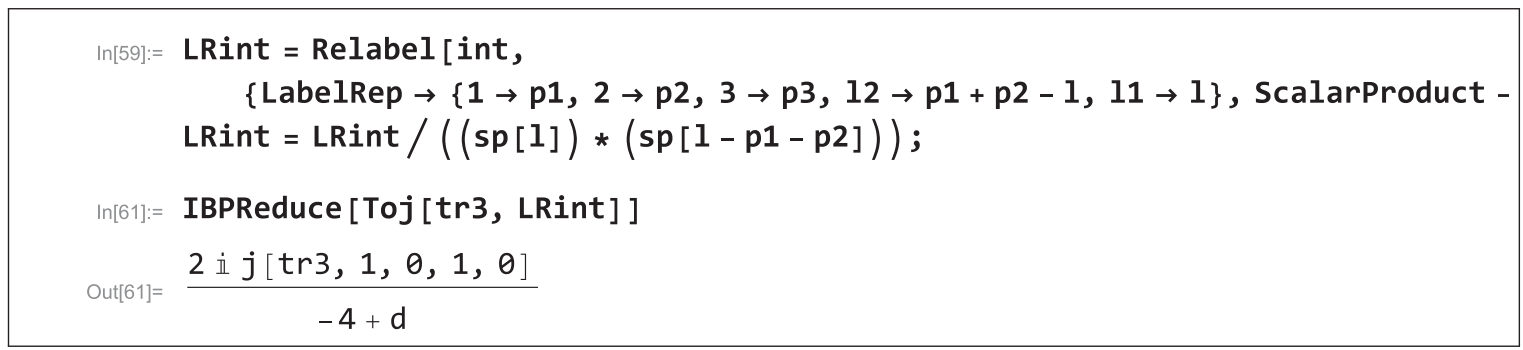

which of course matches with the reduction of expression (4.32):

$$
\begin{aligned}
& \operatorname{In}[62]:=\operatorname{IBPReduce}[-2 \text { i } j[\operatorname{tr} 3,1,0,1,0]-I * s 12 * j[\operatorname{tr} 3,1,1,1,0] \text { ] } \\
& \text { Out[62] }=\frac{2 \text { i } j[\operatorname{tr} 3,1,0,1,0]}{-4+d}
\end{aligned}
$$

where

$$
\begin{aligned}
& \ln [64]:=\operatorname{Fromj}[j[\operatorname{tr} 3,1,0,1,0]] \\
& \text { Out[64] }=\frac{1}{1 \cdot 1(1-p 1-p 2) \cdot(1-p 1-p 2)} \\
& \ln [6]]:=\operatorname{Fromj}[j[\operatorname{tr} 3,1,1,1,0]] \\
& \text { Out[65]= } \frac{1}{1 \cdot 1(1-p 1) \cdot(1-p 1)(1-p 1-p 2) \cdot(1-p 1-p 2)}
\end{aligned}
$$

[1] Z. Bern, L. J. Dixon, D. C. Dunbar, and D. A. Kosower, One loop $\mathrm{n}$ point gauge theory amplitudes, unitarity and collinear limits, Nucl. Phys. B425, 217 (1994).

[2] Z. Bern, L. J. Dixon, D. C. Dunbar, and D. A. Kosower, Fusing gauge theory tree amplitudes into loop amplitudes, Nucl. Phys. B435, 59 (1995).

[3] Z. Bern, L. J. Dixon, and D. A. Kosower, On-shell recurrence relations for one-loop QCD amplitudes, Phys. Rev. D 71, 105013 (2005).

[4] Z. Bern, L. J. Dixon, and D. A. Kosower, The last of the finite loop amplitudes in QCD, Phys. Rev. D 72, 125003 (2005).

[5] D. C. Dunbar and W. B. Perkins, Two-loop five-point all plus helicity Yang-Mills amplitude, Phys. Rev. D 93, 085029 (2016).
[6] D. C. Dunbar, G. R. Jehu, and W. B. Perkins, Two-Loop Six Gluon All Plus Helicity Amplitude, Phys. Rev. Lett. 117, 061602 (2016).

[7] W. L. van Neerven, Dimensional regularization of mass and infrared singularities in two loop on-shell vertex functions, Nucl. Phys. B268, 453 (1986).

[8] Z. Bern and A. G. Morgan, Massive loop amplitudes from unitarity, Nucl. Phys. B467, 479 (1996).

[9] A. Brandhuber, S. McNamara, B. J. Spence, and G. Travaglini, Loop amplitudes in pure Yang-Mills from generalised unitarity, J. High Energy Phys. 10 (2005) 011.

[10] C. Anastasiou, R. Britto, B. Feng, Z. Kunszt, and P. Mastrolia, D-dimensional unitarity cut method, Phys. Lett. B 645, 213 (2007). 
[11] C. Anastasiou, R. Britto, B. Feng, Z. Kunszt, and P. Mastrolia, Unitarity cuts and Reduction to master integrals in d dimensions for one-loop amplitudes, J. High Energy Phys. 03 (2007) 111.

[12] W. T. Giele, Z. Kunszt, and K. Melnikov, Full one-loop amplitudes from tree amplitudes, J. High Energy Phys. 04 (2008) 049.

[13] R. K. Ellis, W. T. Giele, Z. Kunszt, and K. Melnikov, Masses, fermions and generalized $D$-dimensional unitarity, Nucl. Phys. B822, 270 (2009).

[14] Z. Bern, J. J. Carrasco, T. Dennen, Y.-t. Huang, and H. Ita, Generalized unitarity and six-dimensional helicity, Phys. Rev. D 83, 085022 (2011).

[15] R. Boughezal, K. Melnikov, and F. Petriello, The fourdimensional helicity scheme and dimensional reconstruction, Phys. Rev. D 84, 034044 (2011).

[16] S. Davies, One-loop QCD and Higgs to partons processes using six-dimensional helicity and generalized unitarity, Phys. Rev. D 84, 094016 (2011).

[17] C. Cheung and D. O'Connell, Amplitudes and spinorhelicity in six dimensions, J. High Energy Phys. 07 (2009) 075.

[18] S. Badger, H. Frellesvig, and Y. Zhang, A two-loop fivegluon helicity amplitude in QCD, J. High Energy Phys. 12 (2013) 045.

[19] F. R. Anger and V. Sotnikov, On the dimensional regularization of QCD helicity amplitudes with quarks, arXiv: 1803.11127.

[20] S. Abreu, F. Febres Cordero, H. Ita, B. Page, and V. Sotnikov, Planar two-loop five-parton amplitudes from numerical unitarity, J. High Energy Phys. 11 (2018) 116.

[21] S. Badger, C. Brønnum-Hansen, H. B. Hartanto, and T. Peraro, Analytic helicity amplitudes for two-loop five-gluon scattering: The single-minus case, J. High Energy Phys. 01 (2019) 186.

[22] S. Abreu, J. Dormans, F. Febres Cordero, H. Ita, and B. Page, Analytic Form of Planar Two-Loop Five-Gluon Scattering Amplitudes in QCD, Phys. Rev. Lett. 122, 082002 (2019).

[23] F. Wilczek, Decays of Heavy Vector Mesons Into Higgs Particles, Phys. Rev. Lett. 39, 1304 (1977).

[24] M. A. Shifman, A. I. Vainshtein, M. B. Voloshin, and V. I. Zakharov, Low-energy theorems for Higgs boson couplings to photons, Yad. Fiz., 30, 1368 (1979) [Sov. J. Nucl. Phys. 30, 711 (1979)].

[25] W. Buchmuller and D. Wyler, Effective Lagrangian analysis of new interactions and flavor conservation, Nucl. Phys. B268, 621 (1986).

[26] S. Dawson, Radiative corrections to Higgs boson production, Nucl. Phys. B359, 283 (1991).

[27] D. Neill, Two-loop matching onto dimension eight operators in the Higgs-glue sector, arXiv:0908.1573.

[28] D. Neill, Analytic virtual corrections for Higgs transverse momentum spectrum at $\mathrm{O}(\mathrm{alpha}(\mathrm{s}) * * 2 / \mathrm{m}(\mathrm{t}) * * 3)$ via unitarity methods, arXiv:0911.2707.

[29] R. V. Harlander and T. Neumann, Probing the nature of the Higgs-gluon coupling, Phys. Rev. D 88, 074015 (2013).

[30] S. Dawson, I. M. Lewis, and M. Zeng, Effective field theory for Higgs boson plus jet production, Phys. Rev. D 90, 093007 (2014).
[31] F. Cachazo, P. Svrcek, and E. Witten, MHV vertices and tree amplitudes in gauge theory, J. High Energy Phys. 09 (2004) 006.

[32] A. Brandhuber, B. J. Spence, and G. Travaglini, One-loop gauge theory amplitudes in $N=4$ super Yang-Mills from MHV vertices, Nucl. Phys. B706, 150 (2005).

[33] L. J. Dixon, E. W. N. Glover, and V. V. Khoze, MHV rules for Higgs plus multi-gluon amplitudes, J. High Energy Phys. 12 (2004) 015.

[34] S. D. Badger, E. W. N. Glover, and V. V. Khoze, MHV rules for Higgs plus multi-parton amplitudes, J. High Energy Phys. 03 (2005) 023.

[35] S. D. Badger, E. W. N. Glover, and K. Risager, One-loop phi-MHV amplitudes using the unitarity bootstrap, J. High Energy Phys. 07 (2007) 066.

[36] S. Badger, E. W. Nigel Glover, P. Mastrolia, and C. Williams, One-loop Higgs plus four gluon amplitudes: Full analytic results, J. High Energy Phys. 01 (2010) 036.

[37] A. Brandhuber, M. Kostacinska, B. Penante, and G. Travaglini, Higgs Amplitudes from $\mathcal{N}=4$ Super YangMills Theory, Phys. Rev. Lett. 119, 161601 (2017).

[38] Q. Jin and G. Yang, Analytic Two-Loop Higgs Amplitudes in Effective Field Theory and the Maximal Transcendentality Principle, Phys. Rev. Lett. 121, 101603 (2018).

[39] A. Brandhuber, M. Kostacinska, B. Penante, and G. Travaglini, $\operatorname{Tr}\left(F^{3}\right)$ supersymmetric form factors and maximal transcendentality Part I: $\mathcal{N}=4$ super Yang-Mills, J. High Energy Phys. 12 (2018) 076.

[40] A. Brandhuber, M. Kostacinska, B. Penante, and G. Travaglini, $\operatorname{Tr}\left(F^{3}\right)$ supersymmetric form factors and maximal transcendentality Part II: $0<\mathcal{N}<4$ super Yang-Mills, J. High Energy Phys. 12 (2018) 077.

[41] Q. Jin and G. Yang, Hidden analytic relations for two-loop Higgs amplitudes in QCD, arXiv:1904.07260.

[42] Z. Bern, L. J. Dixon, and D. A. Kosower, One loop amplitudes for $e^{+} e^{-}$to four partons, Nucl. Phys. B513, 3 (1998).

[43] R. Britto, F. Cachazo, and B. Feng, Generalized unitarity and one-loop amplitudes in $N=4$ super-Yang-Mills, Nucl. Phys. B725, 275 (2005).

[44] A. Brandhuber, B. Spence, G. Travaglini, and G. Yang, Form factors in $N=4$ super Yang-Mills and periodic Wilson loops, J. High Energy Phys. 01 (2011) 134.

[45] A. Brandhuber, O. Gurdogan, R. Mooney, G. Travaglini, and G. Yang, Harmony of super form factors, J. High Energy Phys. 10 (2011) 046.

[46] T. Gehrmann, J. M. Henn, and T. Huber, The three-loop form factor in $N=4$ super Yang-Mills, J. High Energy Phys. 03 (2012) 101.

[47] A. Brandhuber, G. Travaglini, and G. Yang, Analytic twoloop form factors in $N=4$ SYM, J. High Energy Phys. 05 (2012) 082; J. Phys. Conf. Ser. 523, 012059 (2014).

[48] B. Penante, B. Spence, G. Travaglini, and C. Wen, On super form factors of half-BPS operators in $N=4$ super YangMills, J. High Energy Phys. 04 (2014) 083.

[49] A. Brandhuber, B. Penante, G. Travaglini, and C. Wen, The last of the simple remainders, J. High Energy Phys. 08 (2014) 100.

[50] D. Nandan, C. Sieg, M. Wilhelm, and G. Yang, Cutting through form factors and cross sections of non-protected 
operators in $\mathcal{N}=4$ SYM, J. High Energy Phys. 06 (2015) 156.

[51] F. Loebbert, D. Nandan, C. Sieg, M. Wilhelm, and G. Yang, On-shell methods for the two-loop dilatation operator and finite remainders, J. High Energy Phys. 10 (2015) 012.

[52] A. Brandhuber, M. Kostacinska, B. Penante, G. Travaglini, and D. Young, The SU(2|3) dynamic two-loop form factors, J. High Energy Phys. 08 (2016) 134.

[53] F. Loebbert, C. Sieg, M. Wilhelm, and G. Yang, Two-loop SL(2) form factors and maximal transcendentality, J. High Energy Phys. 12 (2016) 090.

[54] L. Bianchi, A. Brandhuber, R. Panerai, and G. Travaglini, Form factor recursion relations at loop level, J. High Energy Phys. 02 (2019) 182.

[55] L. Bianchi, A. Brandhuber, R. Panerai, and G. Travaglini, Dual conformal invariance for form factors, J. High Energy Phys. 02 (2019) 134.

[56] D. Maitre and P. Mastrolia, S@M, a Mathematica implementation of the spinor-helicity formalism, Comput. Phys. Commun. 179, 501 (2008).

[57] L. J. Dixon, J. M. Henn, J. Plefka, and T. Schuster, All treelevel amplitudes in massless QCD, J. High Energy Phys. 01 (2011) 035.

[58] R. Panerai, Spinor helicity., https://github.com/rpanerai/ Mathematica-Tools (2019).

[59] Z. Bern and D. A. Kosower, The computation of loop amplitudes in gauge theories, Nucl. Phys. B379, 451 (1992).

[60] Z. Bern, A. De Freitas, L. J. Dixon, and H. L. Wong, Supersymmetric regularization, two loop QCD amplitudes and coupling shifts, Phys. Rev. D 66, 085002 (2002).

[61] G. 't Hooft and M. J. G. Veltman, Regularization and renormalization of gauge fields, Nucl. Phys. B44, 189 (1972).

[62] T. Dennen, Y.-t. Huang, and W. Siegel, Supertwistor space for 6D maximal super Yang-Mills, J. High Energy Phys. 04 (2010) 127.

[63] R. Britto, F. Cachazo, B. Feng, and E. Witten, Direct Proof of Tree-Level Recursion Relation in Yang-Mills Theory, Phys. Rev. Lett. 94, 181602 (2005).

[64] S. Dawson, I. M. Lewis, and M. Zeng, Usefulness of effective field theory for boosted Higgs production, Phys. Rev. D 91, 074012 (2015).

[65] L. J. Dixon and Y. Shadmi, Testing gluon self-interactions in three jet events at hadron colliders, Nucl. Phys. B423, 3 (1994); Erratum, Nucl. Phys. B452, 724 (1995).

[66] J. Broedel and L. J. Dixon, Color-kinematics duality and double-copy construction for amplitudes from higherdimension operators, J. High Energy Phys. 10 (2012) 091.

[67] A. Yu. Morozov, Matrix of mixing of scalar and vector mesons of dimension D $<=8$ in QCD , Yad. Fiz., 40, 788 (1984) [Sov. J. Nucl. Phys. 40, 505 (1984)].
[68] J. A. Gracey, Classification and one loop renormalization of dimension-six and dimension-eight operators in quantum gluodynamics, Nucl. Phys. B634, 192 (2002); Erratum, Nucl. Phys. B696, 295 (2004).

[69] M. B. Green and J.H. Schwarz, Supersymmetrical dual string theory. 2. Vertices and trees, Nucl. Phys. B198, 252 (1982).

[70] J. H. Schwarz, Superstring theory, Phys. Rep. 89, 223 (1982).

[71] A. A. Tseytlin, Vector field effective action in the open superstring theory, Nucl. Phys. B276, 391 (1986); Erratum, B291, 876 (1987).

[72] C. R. Schmidt, H $\rightarrow$ ggg (g q anti-q) at two loops in the large M(t) limit, Phys. Lett. B 413, 391 (1997).

[73] R. Mertig, M. Bohm, and A. Denner, FEYN CALC: Computer algebraic calculation of Feynman amplitudes, Comput. Phys. Commun. 64, 345 (1991).

[74] V. Shtabovenko, R. Mertig, and F. Orellana, New developments in FeynCalc 9.0, Comput. Phys. Commun. 207, 432 (2016).

[75] E. D'Hoker and D. Z. Freedman, Supersymmetric gauge theories and the AdS/CFT correspondence, in Proceedings of Strings, Branes and Extra Dimensions: TASI 2001 (2002), pp. 3-158, https://arxiv.org/abs/hep-th/0201253.

[76] P. De Causmaecker, R. Gastmans, W. Troost, and T. T. Wu, Multiple Bremsstrahlung in gauge theories at high-energies. 1. General formalism for quantum electrodynamics, Nucl. Phys. B206, 53 (1982).

[77] F. A. Berends, R. Kleiss, P. De Causmaecker, R. Gastmans, W. Troost, and T. T. Wu, Multiple bremsstrahlung in gauge theories at high-energies. 2. Single bremsstrahlung, Nucl. Phys. B206, 61 (1982).

[78] R. Kleiss and W. J. Stirling, Spinor techniques for calculating $\mathrm{p}$ anti-p $\longrightarrow \mathrm{W}+-/ Z 0+$ jets, Nucl. Phys. B262, 235 (1985).

[79] Z. Xu, D.-H. Zhang, and L. Chang, Helicity amplitudes for multiple Bremsstrahlung in massless nonabelian gauge theories, Nucl. Phys. B291, 392 (1987).

[80] D. A. Kosower, Next-to-maximal helicity violating amplitudes in gauge theory, Phys. Rev. D 71, 045007 (2005).

[81] N. Arkani-Hamed, T.-C. Huang, and Y.-t. Huang, Scattering amplitudes for all masses and spins, arXiv:1709.04891.

[82] A. Brandhuber, D. Korres, D. Koschade, and G. Travaglini, One-loop amplitudes in six-dimensional $(1,1)$ theories from generalised unitarity, J. High Energy Phys. 02 (2011) 077.

[83] M. Accettulli Huber and S. De Angelis, SpinorHelicity6D, https://github.com/accettullihuber/SpinorHelicity (2019).

[84] R. N. Lee, Presenting LiteRed: A tool for the loop integrals reduction, arXiv:1212.2685.

[85] R. N. Lee, LiteRed 1.4: A powerful tool for reduction of multiloop integrals, J. Phys. Conf. Ser. 523, 012059 (2014). 